\title{
Denotational Semantics with Nominal Scott Domains
}

\author{
STEFFEN LÖSCH and ANDREW M. PITTS, University of Cambridge
}

When defining computations over syntax as data, one often runs into tedious issues concerning $\alpha$ equivalence and semantically correct manipulations of binding constructs. Here we study a semantic framework in which these issues can be dealt with automatically by the programming language. We take the user-friendly 'nominal' approach in which bound objects are named. In particular, we develop a version of Scott domains within nominal sets and define two programming languages whose denotational semantics are based on those domains. The first language, $\lambda \nu$-PCF, is an extension of Plotkin's PCF with names that can be swapped, tested for equality and locally scoped; although simple, it already exposes most of the semantic subtleties of our approach. The second language, PNA, extends the first with name abstraction and concretion so that it can be used for metaprogramming over syntax with binders.

For both languages, we prove a full abstraction result for nominal Scott domains analogous to Plotkin's classic result about PCF and conventional Scott domains: two program phrases have the same observable operational behaviour in all contexts if and only if they denote equal elements of the nominal Scott domain model. This is the first full abstraction result we know of for languages combining higher-order functions with some form of locally scoped names which uses a domain theory based on ordinary extensional functions, rather than using the more intensional approach of game semantics.

To obtain full abstraction, we need to add two functionals, one for existential quantification over names and one for 'definite description' over names. Only adding one of them is not enough, as we give counter examples to full abstraction in both cases.

Categories and Subject Descriptors: F.3.2 [Logics and Meanings of Programs]: Semantics of Programming Languages-Denotational semantics; F.4.1 [Mathematical Logic and Formal Languages]: Mathematical Logic_Lambda calculus and related systems

General Terms: Languages, Theory

Additional Key Words and Phrases: Metaprogramming, denotational semantics, domain theory, full abstraction, nominal sets, symmetry

ACM Reference Format:

Steffen Lösch and Andrew M. Pitts. 2014. Denotational Semantics with Nominal Scott Domains. J. ACM X, X, Article XX ( 2014), 45 pages.

DOI : http://dx.doi.org/10.1145/0000000.0000000

\section{INTRODUCTION}

If one wants to express computations not just with numbers, but also with structures representing the programs of a programming language, or the formulas of a logic, then one is in a setting called metaprogramming. It arises for example in mechanised theorem proving, or in domain specific languages. We distinguish between an object-language and a meta-language: we write algorithms in the meta-language for

This work is supported by a Gates Cambridge Scholarship and the ERC Advanced Grant Events, Causality and Symmetry (ECSYM).

Authors' address: S. Lösch and A. M. Pitts, University of Cambridge Computer Laboratory, 15 JJ Thomson Avenue, Cambridge CB3 0FD, United Kingdom.

Permission to make digital or hard copies of part or all of this work for personal or classroom use is granted without fee provided that copies are not made or distributed for profit or commercial advantage and that copies show this notice on the first page or initial screen of a display along with the full citation. Copyrights for components of this work owned by others than ACM must be honored. Abstracting with credit is permitted. To copy otherwise, to republish, to post on servers, to redistribute to lists, or to use any component of this work in other works requires prior specific permission and/or a fee. Permissions may be requested from Publications Dept., ACM, Inc., 2 Penn Plaza, Suite 701, New York, NY 10121-0701 USA, fax +1 (212) 869-0481, or permissions@acm.org.

(C) 2014 ACM 0004-5411/2014/-ARTXX $\$ 15.00$

DOI : http://dx.doi.org/10.1145/0000000.0000000 
manipulating object-language syntax. For example, if we are using the Coq system [coq.inria.fr] to prove theorems about $\mathrm{C}$ programs, then $\mathrm{C}$ is the object-language and Coq is the meta-language.

Whenever the object-language has name-binding constructs (and most programming languages do), we run into tedious issues regarding $\alpha$-equivalence (renaming of bound names) that programmers like to gloss over, but that have to be dealt with in the design of a meta-language. The informal approach is to use the first-order representation of binding as (bound name, body)-pairs and not carry out tedious formal checks that $\alpha$ equivalence is respected by computations. A common formal approach to representing object-language binders is to use function expressions in the meta-language to represent binding operations. However, the notion of 'function' is not absolute in the way that the notion of 'pair' is. As a result it requires some ingenuity (for example, distinguishing functions-as-data from functions-as-computation [Licata and Harper 2009]) to ensure that a meta-language using this form of representation for binders can conveniently express the wide range of first-order syntax-manipulating algorithms commonly employed in informal practice; see Savary-Belanger et al. [2013], for example.

An alternative approach to this problem, that we follow here, is to devise metalanguages that represent object-language binders using the permutation-based notion of name abstraction [Pitts 2013, Chapter 4]. This approach lies somewhere between the first-order and the functional representation and turns out to have good computational properties [Shinwell et al. 2003; Pitts 2011]. In this paper we will investigate the denotational semantics of name abstraction using a 'symmetry-aware' version of Scott domains [Scott 1982].

Various forms of symmetry are used in many branches of mathematics and computer science. The results in this paper have to do with using symmetry to extend the reach of computation theory from finite data structures and algorithms to ones that, although they are infinite, become finite when quotiented by a suitable notion of symmetry. Our motivating example of such data is the abstract syntax trees for an object-language involving name-binding constructs, since infinitely many abstract syntax trees represent the same expression modulo permuting their bound names. This way of viewing $\alpha$-equivalence via symmetry was the initial stimulus for the development of nominal sets, introduced by Gabbay and Pitts [2002]. They provide a theory for mathematical structures involving atomic names ${ }^{1}$ based on name permutations and the notion of $f$ nite support. We review the concept of nominal set briefly in Section 2, referring the reader to Pitts [2013] for a comprehensive account.

Nominal sets have been used to develop the semantic properties of binders and locally scoped names, with applications to functional and logic programming, to equational logic and rewriting, to type theory and to interactive theorem proving; see Gabbay [2011] and Pitts [2013] for recent surveys. The work by Montanari and Pistore [2000] on the $\pi$-calculus and HD-automata provides a somewhat different application of nominal sets (an independent one, since it uses a notion of 'named set' that only subsequently was shown to be equivalent to nominal sets [Gadducci et al. 2006; Staton 2007]). The use of symmetries of names (of fresh communication channels in this case) to get finite representations of infinitely many states is at the forefront in their work. It has recently been subsumed and generalised in a programme of what one might call 'orbit-finite' automata theory [Tzevelekos 2011; Bojańczyk et al. 2011; Bojańczyk and Lasota 2012; Gabbay and Ciancia 2011; Murawski and Tzevelekos 2012].

In this paper we bring together the 'names and binders' and the 'orbit-finite state space' aspects of nominal sets. We observe that a key concept underlying the automatatheoretic research programme of Bojańczyk et al. [2012], that of being an orbit-finite

\footnotetext{
${ }^{1}$ Names whose only attribute is their identity; Harper [2013, part XII] calls them 'symbols'.
} 
subset, turns out to subsume a notion of topological compactness introduced, for quite different purposes, by Turner and Winskel [2009] in their work on nominal domain theory for concurrency. We explain the connection and use it to develop a version of the classic notion of Scott domain within nominal sets. (Previous work on denotational semantics with nominal sets [Shinwell and Pitts 2005; Turner 2009] has focussed on less sophisticated notions of domain, analogous either to $\omega$-chain complete posets, or to algebraic lattices.) The well-known result of Plotkin [1977] proves that PCF with parallel-or is fully abstract with respect to conventional Scott domains, in the sense that two expressions have equal denotations if and only if they the have the same observable operational behaviour in all contexts. We obtain an analogous result for nominal Scott domains, through adding functionals for existential quantification over names and 'definite description' over names to a programming language for recursively defined higher-order functions with name abstractions and Odersky [1994] style locally scoped names.

\section{Outline of the main results in this paper}

- We show that the notion of finiteness used in Turner-Winskel nominal domain theory coincides with the notion of orbit-finite used by Bojańczyk et al. [2012]. Specifically, we prove (Theorem 4.5) that a finitely supported subset of a nominal set is compact with respect to unions that are uniform-directed in the sense of Turner and Winskel if and only if it is orbit-finite.

- We use orbit-finite subsets to generalise the notion of Scott domain from ordinary sets to nominal sets (Definition 5.1). We prove that the category of nominal Scott domains is cartesian closed (Theorem 5.5), has least fixed points and is closed under forming domains of name abstractions (Theorem 10.1). Although there are infinitely many names, the nominal Scott domain of names has some strong finiteness properties. In particular, we show that the functionals for existential quantification over names and definite description of names denotationally are uniform-compact elements of their function domains (Examples 5.7 and 5.8) and can be given a structural operational semantics.

- We define a language $\lambda \nu$-PCF that extends the well-known language PCF of Plotkin [1977] with names that can be locally scoped, swapped, and tested for equality. $\lambda \nu$-PCF's operational semantics is inspired by [Pitts 2011; Lösch and Pitts 2011]. In particular, it makes use of Odersky [1994] style local names, which have a simple denotational semantics using nominal Scott domains. We show that this semantics is computationally adequate, in the sense that equality of denotations implies that two programs are contextually equivalent for the operational semantics (Theorem 7.5).

- We show by counter examples that the reverse implication of computational adequacy can fail, that is, the denotational semantics is not fully abstract (Theorem 8.4), even if $\lambda \nu$-PCF is extended with either existential quantification over names or definite descriptions of names. However, extending $\lambda \nu$-PCF with both new constructs together, we prove that the nominal Scott domain model is fully abstract for the resulting language: any two expressions are contextually equivalent if and only if they have equal denotations in the model (Theorem 8.5).

- We illustrate how $\lambda \nu$-PCF can be the basis for a metaprogramming language for object-languages with binding operations by adding facilities for computing with name abstractions, together with a type representing $\lambda$-calculus terms. The resulting language, PNA (Programming with Name Abstractions), is given a denotational semantics using nominal Scott domains that is computationally adequate (Theorem 11.2). Furthermore, this denotational semantics is shown to be fully abstract once PNA is extended in the same way that $\lambda \nu$-PCF was extended, that is, with existential quantification over names and definite descriptions of names (Theorem 12.2). 
There are full abstraction results for higher-order functions with local names using the intensional approach of game semantics [Abramsky et al. 2004; Tzevelekos 2008; Laird 2008], but our full abstraction Theorems 8.5 and 12.2 are the first such results we know of that are based on ordinary extensional functions. There is no similar result known for FreshML [Shinwell et al. 2003], which uses generative rather than Oderskystyle local names to implement the features that PNA provides for programming with name abstractions; and yet we believe that PNA (extended with recursive types) is in principle as expressive as FreshML, in view of our previous work [Lösch and Pitts 2011]. Our proof of the full abstraction theorems seems novel compared with other proofs of similar full abstraction results in the literature [Curien 2007]. On the other hand it gives rise to some open problems that we discuss at the end of Section 12, together with a number of possibilities for future work exploiting the use of orbit-finite subsets within nominal domain theory.

\section{Note}

This is a revised and extended version of Lösch and Pitts [2013]. Compared with that article, here we show that most of the difficulty with our original full abstraction result (Theorem 12.2) is already present for the simple extension of PCF with locally scoped names rather than with name abstraction as well (Theorem 8.5). We thank the POPL reviewers for indirectly suggesting this approach and for other helpful comments.

In contrast to that article, here we do not extend $\lambda \nu-\mathrm{PCF}^{+}$explicitly with parallel-or, because it can in fact be expressed in terms of existential quantification over names (Remark 8.3). In addition we resolved the open problem of showing that the nominal Scott domain models of $\lambda \nu$-PCF+the and $\lambda \nu$-PCF+ex fail to be fully abstract, using examples suggested by Tzevelekos (Theorem 8.4), whom we thank for discussions on the topic of the paper.

\section{FINITE SUPPORT}

We are interested in the denotational semantics of programs written in languages featuring names that can be tested for equality and locally scoped by binding constructs. To take symmetry into account, we fix some countably infinite set $\mathbb{A}$, whose elements we call atomic names, and consider finite permutations of $\mathbb{A}$, that is, bijections $\pi: \mathbb{A} \cong \mathbb{A}$ with the property that $\pi a=a$ holds for all but finitely many $a \in \mathbb{A}$. Recall that an action of such permutations $\pi$ on the elements $x$ of a set $X$ is a binary operation, written $(\pi, x) \mapsto \pi \cdot x$ and satisfying id $\cdot x=x$ (where id is the identity permutation) and $\pi^{\prime} \cdot(\pi \cdot x)=\left(\pi^{\prime} \circ \pi\right) \cdot x$ (where _ $\circ_{-}$is composition), for all $x \in X$.

Actions of finite permutations of $\mathbb{A}$ on sets $X$ and $Y$ can be extended to their cartesian product $X \times Y$ by defining for each $x \in X$ and $y \in Y$

$$
\pi \cdot(x, y) \triangleq(\pi \cdot x, \pi \cdot y)
$$

More interestingly, given actions on $X$ and $Y$, we get an action on the set of functions $Y^{X}$ by defining for each $f \in Y^{X}$

$$
\pi \cdot f \triangleq \lambda x \in X \rightarrow \pi \cdot\left(f\left(\pi^{-1} \cdot x\right)\right)
$$

where $\pi^{-1}$ is the inverse of the permutation $\pi$. In particular, taking $Y=2=$ \{true, false\}, a two-element set with trivial action $(\pi \cdot$ true $=$ true, $\pi \cdot$ false $=$ false), we get an action on $2^{X}$ and hence on subsets of $X$. This action of finite permutations on subsets has a simple description: for each $S \subseteq X$

$$
\pi \cdot S=\{\pi \cdot x \mid x \in S\} .
$$

Programs, being finite syntactic objects, only involve finitely many atomic names in their construction; whereas the elements of a set $X$ used to denote program behaviours 
may well be infinite mathematical objects. We wish to limit our attention to infinite behaviours that depend only upon finitely many atomic names, as doing so yields a richer and better behaved theory. We can make precise what it means to 'only depend upon finitely many atomic names' entirely in terms of symmetry, that is, in terms of a given permutation action. An element $x \in X$ is supported by a set $A \subseteq \mathbb{A}$ of atomic names if every permutation $\pi$ that preserves each name in $A$ also preserves $x$ :

$$
((\forall a \in A) \pi a=a) \Rightarrow \pi \cdot x=x .
$$

Definition 2.1 (nominal set). We say that a set $X$ equipped with an action of finite permutations of $\mathbb{A}$ is a nominal set if each of its elements is supported by some finite set of atomic names. In this case one can show (see Pitts [2013, Proposition 2.3] for example) that for each $x \in X$ there is a smallest finite subset of $\mathbb{A}$ supporting $x$, which we write as $\operatorname{supp} x$. The freshness relation $a \# x$ is defined by:

$$
a \# x \triangleq a \notin \operatorname{supp} x \text {. }
$$

Note that since $\operatorname{supp} x$ is a finite set and $\mathbb{A}$ is not, given $x$ we can always find some $a \in \mathbb{A}$ satisfying $a \# x$.

Given a nominal set $X$, the subsets that possess a finite support with respect to the action in (3) are called finitely supported subsets of $X$. Not every subset is finitely supported. For example, when $X=\mathbb{A}$ (with action $\pi \cdot a=\pi a$ ), the only finitely supported subsets are those $S \subseteq \mathbb{A}$ for which either $S$, or $\mathbb{A}-S$ is finite [Pitts 2013, Proposition 2.9]. Similarly, not every function $f \in Y^{X}$ between nominal sets $X$ and $Y$ is finitely supported with respect to the action in (2). Functions with empty support are called equivariant and all such functions satisfy for any finite permutation $\pi$ and $x \in X$ that $f(\pi \cdot x)=\pi \cdot(f x)$.

We write $\mathrm{P}_{\mathrm{fs}} X$ for the collection of all finitely supported subsets of $X$. With the action in (3), this is a nominal set; indeed it is the power object (in the sense of topos theory [Johnstone 2002]) for a model of higher-order logic based on nominal sets. The main difference between this model and the classical one is that it fails to satisfy the Axiom of Choice: see Pitts [2013, Section 2.7]. ${ }^{2}$ As we discuss next, this difference causes nominal domain theory to be something more than just 'classical domain theory carried out in the nominal model of higher-order logic'.

\section{UNIFORM-DIRECTED LEAST UPPER BOUNDS}

In this section we recall the nominal domain theory introduced by Turner and Winskel [2009]. A key idea behind domain theory in general is to give a denotation to a program with potentially infinite behaviour as a limit of approximations. For domain theory based on approximation via a partial order (rather than a metric), limits are least upper bounds of chains (totally ordered subsets), or more generally, least upper bounds of subsets that are directed (every finite set of elements in the subset has an upper bound in the subset). So long as one considers chains of arbitrary (ordinal) length, classically there is no difference between using least upper bounds of chains and using least upper bounds of directed subsets [Markowsky 1976]. However, the equivalence of the two approaches relies on the Axiom of Choice and, as we noted above, that fails to hold for nominal sets. Thus in a nominal version of domain theory, formulating limits in terms of least upper bounds of chains leads to a different notion of domain than does the use of least upper bounds of arbitrary directed subsets (of course, both the chains and the directed subsets should be finitely supported, to make sense nominally).

${ }^{2}$ The associated model of set theory goes back to work in the 1930s by Fraenkel and Mostowski, who devised it specifically to negate the Axiom of Choice; see Gabbay [2011, Remark 2.22]. 
Turner and Winskel provide a compelling reason for preferring the former kind of limit: they show by example that a key notion provided by the nominal approach, the operation of name abstraction, does not always preserve least upper bounds of finitely supported, directed subsets. Example 3.2 gives a simplified version of the WinskelTurner example of the failure of name abstraction to preserve least upper bounds of all finitely supported, directed subsets.

Definition 3.1 (name abstraction). A nominal poset is a nominal set $D$ equipped with a partial order $\sqsubseteq$ that is respected by the action of permutations: $d \sqsubseteq d^{\prime} \Rightarrow \pi \cdot d \sqsubseteq$ $\pi \cdot d^{\prime}$. Given such a $D$, we get a pre-order on $\mathbb{A} \times D$ by defining $(a, d) \sqsubseteq\left(a^{\prime}, d^{\prime}\right)$ to hold whenever we have $\left(\begin{array}{ll}a a^{\prime \prime}\end{array}\right) \cdot d \sqsubseteq\left(\begin{array}{ll}a^{\prime} & a^{\prime \prime}\end{array}\right) \cdot d^{\prime}$ in $D$ for some (or indeed, for any) $a^{\prime \prime} \#\left(a, a^{\prime}, d, d^{\prime}\right)$. (As usual, $\left(a a^{\prime}\right)$ denotes the permutation that swaps $a$ and $a^{\prime}$, leaving all other atomic names fixed.) We write $[\mathbb{A}] D$ for the poset obtained by quotienting $\mathbb{A} \times D$ by the equivalence relation associated with this pre-order, and $\langle a\rangle d$ for the equivalence class of $(a, d)$. Defining a permutation action by $\pi \cdot\langle a\rangle d=\langle\pi a\rangle(\pi \cdot d)$, one can show that $[\mathbb{A}] D$ is also a nominal poset, with $\operatorname{supp}\langle a\rangle d=(\operatorname{supp} d)-\{a\}$. We call the elements of $[\mathbb{A}] D$ name abstractions. They are a generalized form of $\alpha$-equivalence class for elements of $D$ - generalized, because $D$ itself may not consist of concrete syntactic data (we just need to know how name permutations act on its elements).

Example 3.2. For any nominal set $X$, partially ordering the elements of the nominal set $\mathrm{P}_{\mathrm{fs}} X$ of finitely supported subsets of $X$ by inclusion, we get a nominal poset. Consider the case when $X=\mathbb{A}$. Given $a \in \mathbb{A}$, the name abstraction function $\mathrm{P}_{\mathrm{fs}} \mathbb{A} \rightarrow[\mathbb{A}]\left(\mathrm{P}_{\mathrm{fs}} \mathbb{A}\right)$ mapping each $S \in \mathrm{P}_{\mathrm{fs}} \mathbb{A}$ to $\langle a\rangle S$ does not preserve all least upper bounds of finitely supported, directed subsets. For example, consider the directed subset $\mathcal{F} \in \mathrm{P}_{\mathrm{fs}}\left(\mathrm{P}_{\mathrm{fs}} \mathbb{A}\right)$ consisting of all finite sets of atomic names. $\mathcal{F}$ has empty support and its least upper bound $\bigsqcup \mathcal{F}$ is equal to $\mathbb{A}$. However, fixing upon $a \neq a^{\prime}$ in $\mathbb{A}$, one has $\langle a\rangle(\bigsqcup \mathcal{F})=\langle a\rangle \mathbb{A}=\left\langle a^{\prime}\right\rangle \mathbb{A} \nsubseteq\left\langle a^{\prime}\right\rangle(\mathbb{A}-\{a\})$ and one can check that $\bigsqcup\{\langle a\rangle F \mid F \in \mathcal{F}\} \sqsubseteq$ $\left\langle a^{\prime}\right\rangle(\mathbb{A}-\{a\})$ (see Pitts [2013, Proposition 11.5]). So $\langle a\rangle(\bigsqcup \mathcal{F}) \neq \bigsqcup\{\langle a\rangle F \mid F \in \mathcal{F}\}$.

Despite this failure of name abstraction to preserve least upper bounds of arbitrary (finitely supported) directed subsets, we will see (Theorem 10.1) that it does preserve least upper bounds of finitely supported chains. Preservation of limits of approximations by a semantic operation is crucial if it is to be used for giving the denotational semantics of some linguistic construct. So one is naturally led to consider a nominal domain theory based upon the use of least upper bounds of finitely supported chains. However, this does not mean that one has to give up entirely the convenience of using directed sets. This is because closure under least upper bounds of finitely supported chains turns out to be equivalent to closure under least upper bounds of directed sets that are not merely finitely supported, but are uniformly supported in the sense of Turner and Winskel [2009, Definition 1]:

Definition 3.3 (uniform support). Let $X$ be a nominal set. A subset $S \subseteq X$ is uniformly supported if there exists a finite set $A$ of atomic names that supports each $x \in S$. Note that if this is the case, then for all finite permutations $\pi$ satisfying $(\forall a \in A) \pi a=a$ we have $\pi \cdot S=\{\pi \cdot x \mid x \in S\}=\{x \mid x \in S\}=S$; so as well as supporting each element of $S, A$ supports $S$ itself. Thus a uniformly supported subset of $X$ is in particular a finitely supported one.

For example, any finite $A \subseteq_{\mathrm{f}} \mathbb{A}$ is a uniformly supported subset (since $A$ itself is a common finite support for each $a \in A$ ), whereas $\mathbb{A}-A$ is finitely supported (by $A$ ), but not uniformly supported (since any support for each $b \in \mathbb{A}-A$ has to contain $b$ ).

The crucial observation is that finitely supported chains in nominal posets have to be uniformly supported (as usual, a chain in $D$ is a subset that is totally ordered by $\sqsubseteq$ ): 
LEMMA 3.4. [Turner 2009, Lemma 3.4.2.1] In a nominal poset D, every finitely supported chain $C$ is necessarily uniformly supported.

Proof. Suppose that $C$ is supported by the finite set $A \subseteq_{\mathrm{f}} \mathbb{A}$. We will show that $A$ supports each $d \in C$.

For any finite permutation $\pi$, if $(\forall a \in A) \pi a=a$, then $\pi \cdot C=C$ (since $A$ supports $C)$. So if $d \in C$, then $\pi \cdot d \in C$. But $C$ is totally ordered, so either $d \sqsubseteq \pi \cdot d$, or $\pi \cdot d \sqsubseteq d$. In the first case, since $\pi$ is a finite permutation, for sufficiently large $n \in \mathbb{N}$ we have $\pi^{n}=$ id and hence $d \sqsubseteq \pi \cdot d \sqsubseteq \pi^{2} \cdot d \sqsubseteq \cdots \sqsubseteq \pi^{n} \cdot d=\mathrm{id} \cdot d=d$; and therefore $d=\pi \cdot d$ by antisymmetry of $\sqsubseteq$. Similarly, if $\pi \cdot d \sqsubseteq d$, then we also get $\pi \cdot d=d$. So in either case $\pi$ preserves $d$. Since this holds for all $\pi$ satisfying $(\forall a \in A) \pi a=a$, we have that $A$ supports $d$.

Definition 3.5 (udcpo). A uniform-directed subset of a nominal poset $D$ is a subset $S \subseteq D$ that is both uniformly supported and directed (that is, each finite subset of $S$ possesses an upper bound in S). A uniform-directed complete partial order (udcpo) is a nominal poset that has a least upper bound $\bigsqcup S$ for all uniform-directed subsets $S$.

As Turner [2009] points out, using Lemma 3.4, the classic result of Markowsky [1976] can be extended to show that a nominal poset is a udcpo if and only if it has least upper bounds for all finitely supported chains. So in effect udcpos give us a domain theory within the higher-order logic of nominal sets based on chain-completeness. As we will see in Section 10, they also give us access to the name abstraction construct.

We model potentially infinite program behaviours in languages with names using denotations that are uniform-directed least upper bounds of approximations to the behaviour. Each approximation should be finite in a suitable sense. For classical domain theory this amounts to being compact (also known as 'finite' or 'isolated') with respect to directed least upper bounds. By analogy, we have:

Definition 3.6 (uniform compactness). An element $u \in D$ of a udcpo $D$ is uniformcompact if for all uniform-directed subsets $S \subseteq D$ it is the case that $u \sqsubseteq \bigsqcup S \Rightarrow(\exists d \in$ $S) u \sqsubseteq d$. We write KD for the set of uniform-compact elements of $D$. We say that $D$ is an algebraic udcpo if each of its elements is the least upper bound of a uniform-directed subset of $K D . D$ is $\omega$-algebraic if in addition the underlying set of $K D$ is countable.

Recall that a subset of a set is compact with respect to directed least upper bounds (unions) of subsets if and only if it is a finite set. Here we are restricting attention to a smaller class of least upper bounds, the uniform-directed ones. Therefore, one should expect uniform-compactness to be a more liberal notion of finiteness. Indeed, we show in the next section that it corresponds precisely to the notion of orbit-finite subset introduced by Bojańczyk et al. [2012, Section 3]. ${ }^{3}$

\section{ORBIT-FINITE SUBSETS}

The action of finite permutations of $\mathbb{A}$ on the elements of a nominal set $X$ partitions them into orbits: two elements $x$ and $x^{\prime}$ are in the same orbit if $x^{\prime}=\pi \cdot x$ for some finite permutation $\pi$. For example $\mathbb{A}$ itself has just one orbit; $\mathbb{A} \times \mathbb{A}$ has two, namely $\{(a, a) \mid a \in \mathbb{A}\}$ and $\left\{\left(a, a^{\prime}\right) \in \mathbb{A}^{2} \mid a \neq a^{\prime}\right\}$; and in general $\mathbb{A}^{n}$ has finitely many orbits, corresponding to equivalence relations on the finite set $\{0,1, \ldots, n-1\}$. Contrastingly, the nominal set $\mathbb{A}^{*}$ of finite lists of atomic names has infinitely many orbits, since lists of different length cannot be in the same orbit.

${ }^{3}$ The term 'finitary subset' is used in that paper, but in subsequent work the authors use the terminology we have adopted here. 
Remark 4.1 (orbit-finite $=$ finitely presentable). The category-theoretic generalisation of the order-theoretic notion of directed least upper bound is the notion of filtered colimit; and compactness with respect to directed least upper bounds generalises to the notion of an object being finitely presentable (fp): an object $X$ in a (locally small) category $\mathbf{C}$ with filtered colimits is fp if the hom-functor $\mathbf{C}\left(X,{ }_{-}\right): \mathbf{C} \rightarrow$ Set preserves filtered colimits. $\mathrm{C}$ is called locally finitely presentable (lfp) if every object is the filtered colimit of fp objects [Gabriel and Ulmer 1971]. The category Nom of nominal sets and equivariant functions, being a Grothendieck topos, is lfp. Although we will not need the characterisation here, it is worth remarking that Petrişan [2011, Proposition 2.3.7] shows that a nominal set is an fp object of Nom if and only if it is orbit-finite, that is, its set of orbits is finite. See Pitts [2013, Section 5.3].

Definition 4.2 (orbit-finite subsets). A finitely supported subset $S \in \mathrm{P}_{\mathrm{fs}} X$ of a nominal set $X$ is said to be orbit-finite if it is contained in the union of finitely many orbits of $X$. We write $\mathrm{P}_{\text {of }} X$ for the collection of orbit-finite subsets of $X$.

Bojańczyk et al. investigate orbit-finite data structures and algorithms (for a generalised version of nominal sets over any 'Fraïssé symmetry'). Note that an orbit-finite subset may well have infinitely many different elements. For example, $\mathbb{A}$ is an orbitfinite subset of itself. Therefore, in order to compute with orbit-finite subsets one needs an effective presentation of them and of operations upon them. The following notion turns out to give an alternative characterisation of orbit-finite subsets that is suitable for calculation. It was introduced independently by Turner [2009, Definition 3.4.3.2], Gabbay [2009, Section 3.3; 2011, Definition 3.1; Gabbay and Ciancia 2011, Definition 3.1] and Bojańczyk et al. [2012, Section 8], whose 'hull' terminology we adopt here. (See also Ciancia and Montanari [2010, Definition 6.10], whose 'closures' are hulls of the form hull $\operatorname{supp} x-\{a\}\{x\}$.)

Definition 4.3 (hulls). Let $X$ be a nominal set. Given finite subsets $A \subseteq_{\mathrm{f}} \mathbb{A}$ and $F \subseteq_{\mathrm{f}} X$, define $\operatorname{hull}_{A} F \triangleq\{\pi \cdot x \mid \pi \# A \wedge x \in F\}$, where $\pi \# A$ as usual means that $\pi$ and $A$ have disjoint support, which in this case means that the finite permutation $\pi$ and the finite set of atomic names $A$ satisfy $(\forall a \in A) \pi a=a$. We call such sets hulls.

LEMMA 4.4. A subset of a nominal set $X$ is orbit-finite if and only if it is a hull. For all finite subsets $A \subseteq_{\mathrm{f}} \mathbb{A}$ and $F \subseteq_{\mathrm{f}} X$, we have hull ${ }_{A} F \in \mathrm{P}_{\mathrm{of}} X$. Conversely, every $S \in \mathrm{P}_{\text {of }} X$ is of the form hull $l_{\text {supp } S} F$ for some $F \subseteq_{\mathrm{f}} X$.

Proof. We sketch the proof and refer the reader to Pitts [2013, Proposition 5.25] for the details. It is not hard to see that hull $F F$ is supported by $A$ and is contained in a finite union of orbits of $X$, namely the orbits of each $x \in F$. What is less obvious is that every orbit-finite subset is of this form. This follows from a key technical property of hulls, proved independently by Turner [2009, Lemma 3.4.3.5] and Bojańczyk et al. [2012, Lemma 3]:

$$
\left(\forall A \subseteq A^{\prime} \subseteq_{\mathrm{f}} \mathbb{A}\right)\left(\forall F \subseteq_{\mathrm{f}} X\right)\left(\exists F^{\prime} \subseteq_{\mathrm{f}} X\right) \operatorname{hull}_{A} F=\operatorname{hull}_{A^{\prime}} F^{\prime} .
$$

Now if $S$ is orbit-finite, it is finitely supported and contained in the union of the orbits of the elements of some finite set $F \subseteq_{f} X$; in other words $S \subseteq$ hull $_{\emptyset} F$. By (6), there exists $F^{\prime} \subseteq_{\mathrm{f}} X$ with hull $\emptyset=\operatorname{hull}_{\operatorname{supp} S} F^{\prime}$ and from this it follows that $S=\operatorname{hull}_{\text {supp } S}\left(F^{\prime} \cap S\right)$.

This lemma can be used to prove the following theorem that makes the connection between orbit-finite subsets and the notion of uniform-compactness from the previous section. Consider the nominal poset $\mathrm{P}_{\mathrm{fs}} X$ of finitely supported subsets of a nominal 
set $X$, the partial order being subset inclusion. It possesses least upper bounds for all finitely supported subsets, given by union, and hence in particular it is a udcpo.

THEOREM 4.5. An element of the udcpo $\mathrm{P}_{\mathrm{fs}} X$ is uniform-compact if and only if it is an orbit-finite subset of $X$. Every $S \in \mathrm{P}_{\mathrm{fs}_{\mathrm{s}}} X$ is the uniform-directed least upper bound of the orbit-finite subsets contained in and with the same support as $S$. Thus $\mathrm{P}_{\mathrm{fs}_{\mathrm{s}}} X$ is an algebraic udcpo in the sense of Definition 3.6.

Proof. Note that for every $S \in \mathrm{P}_{\mathrm{fs}} X$, since any $F \subseteq_{\mathrm{f}} S$ satisfies $F \subseteq \operatorname{hull}_{\text {supp } S} F \subseteq S$, we have

$$
S=\bigcup\left\{\operatorname{hull}_{\text {supp } S} F \mid F \subseteq_{\mathrm{f}} S\right\}
$$

and the right-hand side is a directed union of orbit-finite subsets that have common support supp $S$. Therefore to prove the theorem it just suffices to prove that any $S$ is uniform-compact if and only if it is orbit-finite.

Suppose $S \in \mathrm{P}_{\text {of }} X$ and $S \subseteq \cup \mathcal{S}$ with $\mathcal{S}$ a uniform-directed subset of $\mathrm{P}_{\mathrm{fs}} X$. By Lemma 4.4, $S=\operatorname{hull}_{A} F$ for some $A \subseteq_{\mathrm{f}} \mathbb{A}$ and $F \subseteq_{\mathrm{f}} X$; and by (6) we may assume that $A$ supports each element of $\mathcal{S}$. Since $F$ is finite, $F \subseteq \bigcup \mathcal{S}$ and $\mathcal{S}$ is directed, we have $F \subseteq S^{\prime}$ for some $S^{\prime} \in \mathcal{S}$; and since $A$ supports $S^{\prime}, F \subseteq S^{\prime}$ implies that hull ${ }_{A} F \subseteq S^{\prime}$, that is, $S \subseteq S^{\prime}$. So $S$ is uniform-compact.

Conversely, if $S \in \mathrm{P}_{\mathrm{fs}} X$ is uniform-compact, then in view of (7) we have $S \subseteq$ hull $_{\text {supp } S} F$ for some $F \subseteq_{\mathrm{f}} S$. Hence $S=\operatorname{hull}_{\text {supp } S} F$ is orbit-finite by Lemma 4.4.

\section{NOMINAL SCOTT DOMAINS}

In view of Theorem 4.5 there is the following analogy

$$
\frac{\text { finite }}{\text { directed }} \text { sets } \sim \frac{\text { orbit-finite }}{\text { uniform-directed }} \text { nominal sets }
$$

which we apply to transfer to nominal sets the classical notion of Scott domain that arose in the denotational semantics of higher-order functional programming languages [Plotkin 1977, Lemma 4.4].

Definition 5.1. A nominal Scott domain $D$ is an $\omega$-algebraic udcpo with a least element and least upper bounds for all finitely supported subsets that have upper bounds (or equivalently, by Theorem 4.5, least upper bounds for all orbit-finite subsets of $K D$ that have upper bounds). Functions between nominal Scott domains are called uniform-continuous if they are finitely supported, monotone, and preserve all uniform-directed least upper bounds. The category Nsd has nominal Scott domains for its objects and for its morphisms it has functions $f: D \rightarrow D^{\prime}$ that are both equivariant and uniform-continuous.

Definition 5.2 (flat domains). If $X$ is a nominal set, the flat nominal poset $X_{\perp}$ is given by $X \uplus\{\perp\}$, with partial order $d \sqsubseteq d^{\prime} \Leftrightarrow d=\perp \vee d=d^{\prime}$ and permutation action extending that on $X$ by $\pi \cdot \perp=\perp$. It is easily seen to be a nominal Scott domain, with $\mathrm{K}\left(X_{\perp}\right)=X_{\perp}$, provided the underlying set of $X$ is countable.

Remark 5.3 (Turner-Winskel domain theory). The nominal domain theory for concurrency of Turner and Winskel [2009] introduces the notion of 'uniform-directed least upper bound' and contains a characterisation of uniform-compact elements in terms of the hull construct from Definition 4.3. However, their domains are more specific than ours since they are based on path sets (downwards-closed subsets of preorders), which form prime-algebraic complete lattices. Modulo countability, their category FMCts $\mathbf{F}_{\emptyset}$ is a full subcategory of Nsd. 
LEMMA 5.4. Let D be a udcpo. If an orbit-finite subset of $\mathrm{KD}$ possesses a least upper bound in $D$, then that least upper bound is also in $\mathrm{K} D$.

PRoof. Suppose $K \in \mathrm{P}_{\text {of }}(\mathrm{K} D)$ possesses a least upper bound and that $\bigsqcup K \sqsubseteq \bigsqcup S$ for some uniform-directed subset $S \subseteq D$. By Lemma 4.4 and (6) we can assume $K=$ hull ${ }_{A} F$ where $F \subseteq_{\mathrm{f}} \mathrm{K} D$ and $A \subseteq_{\mathrm{f}} \mathbb{A}$ supports each $d \in S$. Since $F \subseteq$ hull $_{A} F=K$, we get $(\forall u \in F) u \sqsubseteq \bigsqcup K \sqsubseteq \bigsqcup S$; so since $u$ is uniform-compact, $F$ is finite and $S$ is directed, there is some $d \in \bar{S}$ satisfying $(\forall u \in F) u \sqsubseteq d$. Because $A$ supports $d$, if follows that $\left(\forall u \in \operatorname{hull}_{A} F\right) u \sqsubseteq d$ and therefore $\bigsqcup K=\bigsqcup$ hull ${ }_{A} F \sqsubseteq d$. So we do indeed have $\bigsqcup K \in \mathrm{K} D$.

\section{THEOREM 5.5. Nsd is cartesian closed.}

Proof. The terminal object is given by the trivial flat domain $\emptyset_{\perp}$. The product of $D_{1}$ and $D_{2}$ is given by their cartesian product, with permutation action as in (1) and partial order $\left(d_{1}, d_{2}\right) \sqsubseteq\left(d_{1}^{\prime}, d_{2}^{\prime}\right) \triangleq d_{1} \sqsubseteq d_{1}^{\prime} \wedge d_{2} \sqsubseteq d_{2}^{\prime}$. Exponentials $D_{1} \rightarrow D_{2}$ have an underlying set consisting of all functions $f \in D_{2}^{D_{1}}$ that are uniform-continuous. The partial order on such functions is also given as usual, argument-wise: $f \sqsubseteq f^{\prime} \triangleq(\forall d \in$ $\left.D_{1}\right) f d \sqsubseteq f^{\prime} d$. That $D_{1} \rightarrow D_{2}$ is a udcpo, has a least element, has least upper bounds of all finitely supported bounded subsets, and has the correct universal property for an exponential are all easy to verify. Less straightforward is its $\omega$-algebraicity with respect to uniform-directed least upper bounds: for ordinary Scott domains, compact elements of the exponential are given by least upper bounds of finite, bounded sets of step-functions; here we use orbit-finite rather than finite sets. Given uniform-compact elements $u_{i} \in \mathrm{K} D_{i}(i=1,2)$, consider the step-function

$$
\left(u_{1} \searrow u_{2}\right) \triangleq \lambda d \in D_{1} \rightarrow \text { if } u_{1} \sqsubseteq d \text { then } u_{2} \text { else } \perp .
$$

It is easily seen to be uniform-continuous (because $u_{1}$ is uniform-compact) and uniform-compact (because $u_{2}$ is uniform-compact). Let $\left(D_{1} \rightarrow_{\text {step }} D_{2}\right) \subseteq \mathrm{K}\left(D_{1} \rightarrow D_{2}\right)$ be the set of all such step-functions, and note that this is a countable nominal set with $\pi \cdot\left(u_{1} \searrow u_{2}\right)=\left(\pi \cdot u_{1} \searrow \pi \cdot u_{2}\right)$. For algebraicity of $D_{1} \rightarrow D_{2}$ we show that for each $f \in D_{1} \rightarrow D_{2}$ we have $f=\bigsqcup S_{f}$, where

$$
\begin{aligned}
S_{f} \triangleq\left\{\bigsqcup \operatorname{hull}_{\text {supp } f} F \mid F \subseteq_{\mathrm{f}} K_{f}\right\} \\
K_{f} \triangleq\left\{\left(u_{1} \searrow u_{2}\right) \in\left(D_{1} \rightarrow_{\text {step }} D_{2}\right) \mid\left(u_{1} \searrow u_{2}\right) \sqsubseteq f\right\} .
\end{aligned}
$$

Every hull supp $f_{f} F$ is bounded by $f$ and finitely supported by $\operatorname{supp} f$, so $\bigsqcup$ hull $\operatorname{supp}_{f} F$ exists and is uniform-compact by Lemma 5.4. This shows that $S_{f}$ consists of uniformcompact elements, is bounded by $f$, is uniformly supported by supp $f$ and is directed (as hull $_{A}(-)$ preserves inclusions), so its least upper bound exists and satisfies $\bigsqcup S_{f} \sqsubseteq f$.

To prove $f=\bigsqcup S_{f}$ we still need $f \sqsubseteq \bigsqcup S_{f}$, which by algebraicity of $D_{1}$ holds if $\left(\forall u_{1} \in\right.$ $\left.\mathrm{K} D_{1}\right) f u_{1} \sqsubseteq\left(\bigsqcup S_{f}\right) u_{1}$. For each $u_{1} \in \mathrm{K} D_{1}$, since $D_{2}$ is algebraic, we have $f u_{1}=\bigsqcup U_{2}$ for some uniform-directed subset $U_{2} \subseteq K D_{2}$. For each $u_{2} \in U_{2}, u_{2} \sqsubseteq \bigsqcup U_{2}=f u_{1}$ and hence $\left(u_{1} \searrow u_{2}\right) \sqsubseteq f$. Therefore $\left(u_{1} \searrow u_{2}\right) \in K_{f}$; and since $\left(u_{1} \searrow u_{2}\right) \in \operatorname{hull}_{\operatorname{supp} f}\left\{\left(u_{1} \searrow u_{2}\right)\right\}$ we get $\left(u_{1} \searrow u_{2}\right) \sqsubseteq \bigsqcup$ hull $\operatorname{supp} f\left\{\left(u_{1} \searrow u_{2}\right)\right\} \in S_{f}$. Hence $\left(u_{1} \searrow u_{2}\right) \sqsubseteq \bigsqcup S_{f}$ and thus $u_{2}=\left(u_{1} \searrow u_{2}\right) u_{1} \sqsubseteq\left(\bigsqcup S_{f}\right) u_{1}$. Since this is true for each $u_{2} \in U_{2}$, we get $f u_{1}=\bigsqcup U_{2} \sqsubseteq$ $\left(\bigsqcup S_{f}\right) u_{1}$, as required.

Knowing $f \sqsubseteq \bigsqcup S_{f}$ also allows us to characterise the uniform-compact elements of $D_{1} \rightarrow D_{2}$ by

$$
\mathrm{K}\left(D_{1} \rightarrow D_{2}\right)=\left\{\bigsqcup S \mid S \in \mathrm{P}_{\text {of }}\left(D_{1} \rightarrow_{\text {step }} D_{2}\right) \wedge S \text { is bounded }\right\}
$$

and this shows that $D_{1} \rightarrow D_{2}$ is $\omega$-algebraic, because $\mathrm{P}_{\text {of }} X$ is countable if $X$ is. 
We give some examples of uniform-compact elements of exponential objects in Nsd associated with the flat domain of atomic names, $\mathbb{A}_{\perp}$. The examples show that although $\mathbb{A}_{\perp}$ has a countably infinite underlying set, it has very different uniform-compactness properties from the flat domain of natural numbers, $\mathbb{N}_{\perp}$, for which the permutation action is discrete: $\pi \cdot n \triangleq n$. It turns out that these examples are important for the full abstraction results developed later in this paper.

Example 5.6 (name equality test). Let $2=\{$ true, false $\}$ be a two-element, discrete nominal set. For each atomic name $a \in \mathbb{A}$, consider the function $e q_{a}: \mathbb{A}_{\perp} \rightarrow 2_{\perp}$ given by

$$
e q_{a} d \triangleq \begin{cases}\text { true } & \text { if } d=a \\ \text { false } & \text { if } d \in \mathbb{A}-\{a\} \\ \perp & \text { if } d=\perp\end{cases}
$$

for each $d \in \mathbb{A}_{\perp}$. This function can be expressed in terms of hulls (Definition 4.3) and step-functions (8):

$$
e q_{a}=\bigsqcup \operatorname{hull}_{\{a\}}\left\{(a \searrow \text { true }),\left(a^{\prime} \searrow \text { false }\right)\right\}
$$

where $a^{\prime}$ is any atomic name not equal to $a$. Thus from (9) we have $e q_{a} \in \mathrm{K}\left(\mathbb{A}_{\perp} \rightarrow 2_{\perp}\right)$.

Example 5.7 (exists name). The function exists $_{\mathbb{A}}:\left(\mathbb{A}_{\perp} \rightarrow 2_{\perp}\right) \rightarrow 2_{\perp}$ defined by

$$
\text { exists }_{\mathbb{A}} f \triangleq \begin{cases}\text { true } & \text { if }(\exists a \in \mathbb{A}) f a=\text { true } \\ \text { false } & \text { if }(\forall a \in \mathbb{A}) f a=\text { false } \\ \perp & \text { otherwise. }\end{cases}
$$

is not only uniform-continuous, it is in fact uniform-compact. To see this, first note that picking any $a \in \mathbb{A}$, the least upper bound $\bigsqcup$ hull $\emptyset_{\emptyset}\{(a \searrow$ false $)\}$ exists and by (9) it is an element of $\mathrm{K}\left(\mathbb{A}_{\perp} \rightarrow 2_{\perp}\right)$. Observe that this least upper bound is exactly the function $k_{\text {false }} \triangleq \lambda d \in \mathbb{A}_{\perp} \rightarrow$ if $d=\perp$ then $\perp$ else false and that

$$
\left(\forall f \in \mathbb{A}_{\perp} \rightarrow 2_{\perp}\right) k_{\text {false }} \sqsubseteq f \Leftrightarrow(\forall a \in \mathbb{A}) f a=\text { false } .
$$

Furthermore, for any $g:\left(\mathbb{A}_{\perp} \rightarrow 2_{\perp}\right) \rightarrow 2_{\perp}$ we have exists $s_{\mathbb{A}} \sqsubseteq g$ if and only if $(\forall f \in$ $\left.\mathbb{A}_{\perp} \rightarrow 2_{\perp}\right)$ exists $_{\mathbb{A}} f \sqsubseteq g f$ if and only if

$$
\begin{aligned}
\left(\forall f \in \mathbb{A}_{\perp} \rightarrow 2_{\perp}\right) & ((\exists a \in \mathbb{A}) f a=\text { true }) \Rightarrow g f=\text { true } \\
\wedge & \wedge((\forall a \in \mathbb{A}) f a=\text { false }) \Rightarrow g f=\text { false }
\end{aligned}
$$

which by definition of step-functions (8) and by (12) is equivalent to

$$
\left(\forall f \in \mathbb{A}_{\perp} \rightarrow 2_{\perp}\right)(\forall a \in \mathbb{A})((a \searrow \text { true }) \sqsubseteq f \Rightarrow g f=\text { true }) \wedge\left(k_{\text {false }} \sqsubseteq f \Rightarrow g f=\text { false }\right)
$$

or more simply, to $(\forall a \in \mathbb{A})((a \searrow$ true $) \searrow$ true $) \sqsubseteq g \wedge\left(k_{\text {false }} \searrow\right.$ false $) \sqsubseteq g$. Thus picking some $a \in \mathbb{A}$, we have $\operatorname{exists}_{\mathbb{A}}=\bigsqcup$ hull $\left\{\left\{((a \searrow\right.\right.$ true $) \searrow$ true $),\left(k_{\text {false }} \searrow\right.$ false $\left.)\right\}$ and by (9) we have exists $_{\mathbb{A}} \in \mathrm{K}\left(\left(\mathbb{A}_{\perp} \rightarrow 2_{\perp}\right) \rightarrow 2_{\perp}\right)$.

Example 5.8 (definite name description). The name equality tests $e q_{a}: \mathbb{A}_{\perp} \rightarrow 2_{\perp}$ from Example 5.6 satisfy

$$
\begin{gathered}
\pi \cdot e q_{a}=e q_{\pi a} \\
e q_{a}=e q_{a^{\prime}} \Rightarrow a=a^{\prime} \\
e q_{a} \sqsubseteq f \Rightarrow e q_{a}=f .
\end{gathered}
$$


From these properties and the uniform-compactness of $e q_{a}$ it follows that the function the $e_{\mathbb{A}}:\left(\mathbb{A}_{\perp} \rightarrow 2_{\perp}\right) \rightarrow \mathbb{A}_{\perp}$ defined by

$$
\text { the }_{\mathbb{A}} f \triangleq \begin{cases}a & \text { if } f=e q_{a} \text { for some } a \in \mathbb{A} \\ \perp & \text { otherwise }\end{cases}
$$

satisfies for all $g:\left(\mathbb{A}_{\perp} \rightarrow 2_{\perp}\right) \rightarrow \mathbb{A}_{\perp}$ that the $e_{\mathbb{A}} \sqsubseteq g$ holds if and only if $(\forall a \in \mathbb{A})\left(e q_{a} \searrow a\right) \sqsubseteq g$. Therefore picking any $a \in \mathbb{A}$, we have the $e_{\mathbb{A}}=\bigsqcup \operatorname{hull}_{\emptyset}\left\{\left(e q_{a} \searrow a\right)\right\}$ and hence by (9) that the $e_{\mathbb{A}} \in \mathrm{K}\left(\left(\mathbb{A}_{\perp} \rightarrow 2_{\perp}\right) \rightarrow \mathbb{A}_{\perp}\right)$.

Remark 5.9. The analogue of the equality test function of Example 5.6 for natural numbers, $e q_{n}: \mathbb{N}_{\perp} \rightarrow 2_{\perp}$, is not uniform-compact. To see this, note that it can be expressed as the least upper bound of a finitely supported chain of functions $e q_{n}=\bigsqcup\left\{e q_{n, m} \mid m \in \mathbb{N}\right\}$, where $e q_{n, m}$ is defined by

$$
e q_{n, m} d \triangleq \begin{cases}e q_{n} d & \text { if } d=m^{\prime} \wedge m^{\prime} \leq m \\ \perp & \text { otherwise }\end{cases}
$$

If $e q_{n}$ was uniform-compact, then $e q_{n} \sqsubseteq e q_{n, m}$ would hold for some $m \in \mathbb{N}$, which is not the case. We cannot use the same kind of argument for $e q_{a}$, because there is no finitely supported total ordering of the elements of $\mathbb{A}$.

The functionals for existential quantification and definite description of natural numbers exist $_{\mathbb{N}}$ and the $e_{\mathbb{N}}$ not only fail to be uniform-compact, they are also not uniform-continuous. For the $e_{\mathbb{N}}, e q_{n}$ from above gives a counter-example to uniformcontinuity, because the $e_{\mathbb{N}}\left(e q_{n}\right)=n$ as well as $(\forall m \in \mathbb{N})$ the $e_{\mathbb{N}}\left(e q_{n, m}\right)=\perp$ hold. For exists $s_{\mathbb{N}}$, observe that $k_{\text {false }}$ from Example 5.7 (for numbers instead of names, so $k_{\text {false }}: \mathbb{N}_{\perp} \rightarrow 2_{\perp}$ ) can be characterised by $k_{\text {false }}=\bigsqcup\left\{k_{\text {false }, m} \mid m \in \mathbb{N}\right\}$, where

$$
k_{\text {false }, m} d \triangleq \begin{cases}\text { false } & \text { if } d=m^{\prime} \wedge m^{\prime} \leq m \\ \perp & \text { otherwise. }\end{cases}
$$

This contradicts uniform-continuity of exists $\mathbb{N}_{\mathbb{N}}$, as $\operatorname{exists}_{\mathbb{N}}\left(k_{\text {false }}\right)=$ false and $(\forall m \in$ $\mathbb{N}) \operatorname{exist}_{\mathbb{N}}\left(k_{\mathrm{false}, m}\right)=\perp$.

Remark 5.10 (least fixed points). If $D \in \mathbf{N s d}$ and $f \in D \rightarrow D$, then $\left\{\perp, f \perp, f^{2} \perp, \ldots\right\}$ is a uniform-directed subset of $D$ (each element is supported by supp $f$ ) whose least upper bound is fix $f$, the least fixed point of $f$, by the usual Tarskian argument. Indeed for each nominal Scott domain $D$, the function assigning least fixed points gives us a morphism in Nsd

$$
\text { fix : }(D \rightarrow D) \rightarrow D \text {. }
$$

\section{SEMANTICS OF LOCALLY SCOPED NAMES}

As for any cartesian closed category, Theorem 5.5 allows one to model the typed $\lambda$ calculus using the category Nsd; and in view of Remark 5.10, Nsd also supports the usual domain-theoretic interpretation of recursively defined terms via least fixed points. For example, nominal Scott domains subsume the usual Scott semantics of Plotkin's language PCF [Plotkin 1977] for recursively defined higher-order numerical functions. At the same time Nsd can give denotational semantics for programming language features involving atomic names, such as local scoping and abstraction; furthermore this denotational semantics is pleasingly simple compared with the use of functor categories, for example by Stark [1994, Chapter 3]. We postpone discussing name abstraction until Section 9 and concentrate first on the Nsd semantics of locally scoped names. 


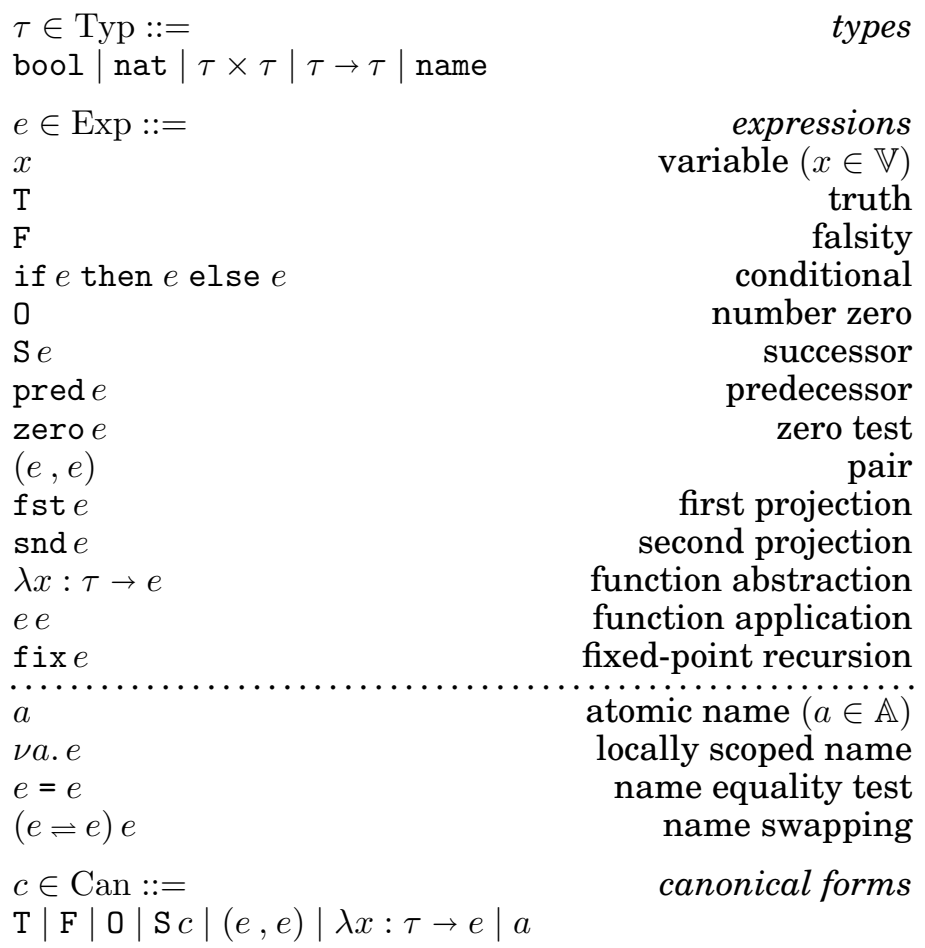

Fig. 1. Syntax of $\lambda \nu$-PCF

To do so, we consider a combination of Plotkin's PCF with Odersky's $\lambda \nu$-calculus, a functional theory of local names [Odersky 1994]. The resulting programming language, which we call $\lambda \nu$-PCF, not only has arithmetic constructs and (call-by-name, higherorder) recursive functions, but also has a type of atomic names that can be tested for equality, locally scoped and explicitly swapped (in expressions of any type).

Figure 1 gives the syntax of $\lambda \nu$-PCF. In the grammar for expressions, the part below the dotted line is what is added to PCF. There are two kinds of identifier in the language: variables $x, y, z, f, \ldots \in \mathbb{V}$ and atomic names $a, b, c, \ldots \in \mathbb{A}$. The sets $\mathbb{V}$ of variables and $\mathbb{A}$ of atomic names are disjoint and countably infinite. Both kinds of identifier may be bound; binding forms are function abstraction $\lambda x: \tau \rightarrow_{-}$(for variables) and local scoping $\nu a$. $_{\text {- }}$ (for atomic names). We identify expressions up to $\alpha$-equivalence of bound identifiers. For any expression $e$, we write $\mathrm{fn}(e)$ for its finite set of free atomic names and $\mathrm{fv}(e)$ for its finite set of free variables.

The reason for making a syntactic distinction between variables $x$ and atomic names $a$ is that they behave differently: an occurrence of $x$ in an expression stands for an unknown expression; whereas an occurrence of $a$ denotes an entity whose identity is definitely different from the other atomic names that occur in the expression. Thus if $x$ and $y$ are two different variables, the meaning of the boolean expression $x=y$ is indeterminate, whereas if $a$ and $b$ are two different atomic names, then the meaning of $a=b$ is the boolean value false. For this reason various properties of $\lambda \nu-\mathrm{PCF}$, such as its typing judgement, are preserved by the operation of substitution of expressions for variables, but are only preserved by permutations of atomic names rather than more general forms of substitution for names. The operation of simultaneous substitution 


$$
\begin{aligned}
& \frac{(x: \tau) \in \Gamma}{\Gamma \vdash x: \tau} \quad \frac{c \in\{\mathrm{T}, \mathrm{F}\}}{\Gamma \vdash c: \text { bool }} \quad \frac{\Gamma \vdash e_{1}: \text { bool } \quad \Gamma \vdash e_{2}: \tau \quad \Gamma \vdash e_{3}: \tau}{\Gamma \vdash \text { if } e_{1} \text { then } e_{2} \text { else } e_{3}: \tau} \quad \frac{}{\Gamma \vdash 0: \text { nat }} \\
& \frac{\Gamma \vdash e: \text { nat }}{\Gamma \vdash \mathrm{S} e: \text { nat }} \quad \frac{\Gamma \vdash e: \text { nat }}{\Gamma \vdash \text { pred } e: \text { nat }} \quad \frac{\Gamma \vdash e: \text { nat }}{\Gamma \vdash \text { zeroe }: \text { bool }} \quad \frac{\Gamma \vdash e_{1}: \tau_{1} \Gamma \vdash e_{2}: \tau_{2}}{\Gamma \vdash\left(e_{1}, e_{2}\right): \tau_{1} \times \tau_{2}} \\
& \frac{\Gamma \vdash e: \tau_{1} \times \tau_{2}}{\Gamma \vdash \text { fst } e: \tau_{1}} \quad \frac{\Gamma \vdash e: \tau_{1} \times \tau_{2}}{\Gamma \vdash \text { snd } e: \tau_{2}} \quad \frac{\Gamma, x: \tau \vdash e: \tau^{\prime}}{\Gamma \vdash \lambda x: \tau \rightarrow e: \tau \rightarrow \tau^{\prime}} \\
& \frac{\Gamma \vdash e_{1}: \tau \rightarrow \tau^{\prime} \quad \Gamma \vdash e_{2}: \tau}{\Gamma \vdash e_{1} e_{2}: \tau^{\prime}} \quad \frac{\Gamma \vdash e: \tau \rightarrow \tau}{\Gamma \vdash \mathrm{fix} e: \tau} \\
& \frac{a \in \mathbb{A}}{\Gamma \vdash a: \text { name }} \quad \frac{a \in \mathbb{A} \quad \Gamma \vdash e: \tau}{\Gamma \vdash \nu a . e: \tau} \quad \frac{\Gamma \vdash e_{1}: \text { name } \Gamma \vdash e_{2}: \text { name }}{\Gamma \vdash e_{1}=e_{2}: \text { bool }} \\
& \frac{\Gamma \vdash e_{1}: \text { name } \quad \Gamma \vdash e_{2}: \text { name } \quad \Gamma \vdash e_{3}: \tau}{\Gamma \vdash\left(e_{1} \rightleftharpoons e_{2}\right) e_{3}: \tau}
\end{aligned}
$$

Fig. 2. $\lambda \nu$-PCF typing rules

of expressions $e_{1}, \ldots, e_{n}$ for distinct variables $x_{1}, \ldots, x_{n}$ in an expression $e$ is written as $e\left[e_{1} / x_{1}, \ldots, e_{n} / x_{n}\right]$, where the substitution avoids capture of both free variables and free atomic names by the language's binding forms. The operation of applying a finite permutation $\pi: \mathbb{A} \cong \mathbb{A}$ to an expression $e$ is written $\pi \cdot e$. It is defined by recursing into all sub-expressions and applying $\pi$ to all occurrences of atomic names. This is a permutation action in the sense of Section 2 and makes the set Exp of $\lambda \nu$-PCF expressions into a nominal set. Since the elements of Exp are expressions up to $\alpha$-equivalence, the smallest support of an element $e$ of this nominal set of expressions is given by its free names fn(e); see Pitts [2013, Proposition 8.10].

The language $\lambda \nu$-PCF contains one syntactic form absent from Odersky's $\lambda \nu$-calculus: explicit swapping expressions, $\left(e_{1} \rightleftharpoons e_{2}\right) e$. Recall that every finite permutation is a composition of transpositions $\left(a a^{\prime}\right)$, the permutation that swaps $a$ and $a^{\prime}$ while leaving every other atomic name fixed. It follows that the operation on expressions mapping $e$ to $\pi \cdot e$ can be expressed in terms of the operations $e \mapsto\left(a a^{\prime}\right) \cdot e$ for all $a, a^{\prime} \in \mathbb{A}$. The language $\lambda \nu$-PCF internalizes this name-swapping operation with expressions of the form $\left(e_{1} \rightleftharpoons e_{2}\right) e$, where $e_{1}$ and $e_{2}$ are expressions of type name that need not be specific atomic names (for example, they could be variables of type name). These expressions seem quite natural in the context of semantics based on nominal sets and we use them in the full abstraction proof in Section 8.

Definition 6.1 ( $\lambda \nu$-PCF typing). $\quad \lambda \nu$-PCF is a simply typed language. The grammar for types (Figure 1) extends that for PCF (in a version with products $\tau_{1} \times \tau_{2}$ ) with a type name of atomic names. The inductively defined typing judgement $\Gamma \vdash e: \tau$ (read as 'in the environment $\Gamma$ the expression $e$ has type $\tau$ ') is defined in Figure 2 by the usual rules for PCF and, below the dotted line, rules concerning names. The typing environments $\Gamma=\left\{x_{1}: \tau_{1}, \ldots, x_{n}: \tau_{n}\right\}$ are finite functions from variables to types that track occurrences of free variables in $e$. For simplicity, $\lambda \nu$-PCF only features one sort of atomic name (many-sorted versions are possible; cf. Pitts [2013, Section 4.7]). 


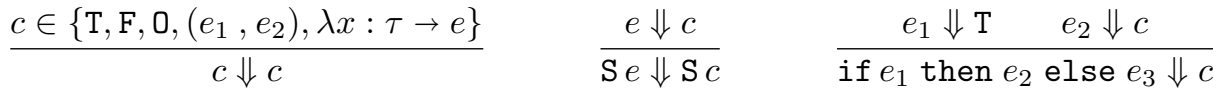

$$
\begin{aligned}
& \frac{e_{1} \Downarrow \mathrm{F} \quad e_{3} \Downarrow c}{\text { if } e_{1} \text { then } e_{2} \text { else } e_{3} \Downarrow c} \quad \frac{e \Downarrow \mathrm{S} c}{\operatorname{pred} e \Downarrow c} \quad \frac{e \Downarrow 0}{\text { zero } e \Downarrow \mathrm{T}} \quad \frac{e \Downarrow \mathrm{S} c}{\text { zero } e \Downarrow \mathrm{F}} \\
& \frac{e \Downarrow\left(e_{1}, e_{2}\right) \quad e_{1} \Downarrow c}{\mathrm{fst} e \Downarrow c} \quad \frac{e \Downarrow\left(e_{1}, e_{2}\right) \quad e_{2} \Downarrow c}{\operatorname{snd} e \Downarrow c} \quad \frac{\begin{array}{c}
e_{1} \Downarrow \lambda x: \tau \rightarrow e \\
e\left[e_{2} / x\right] \Downarrow c
\end{array}}{e_{1} e_{2} \Downarrow c} \quad \frac{e(\mathrm{fix} e) \Downarrow c}{\mathrm{fix} e \Downarrow c}
\end{aligned}
$$

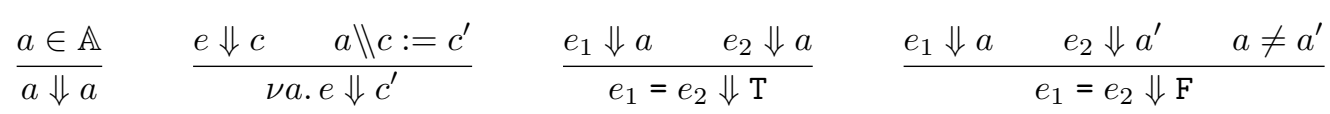

$$
\begin{aligned}
& \frac{e_{1} \Downarrow a_{1} \quad e_{2} \Downarrow a_{2} \quad e_{3} \Downarrow c}{\left(e_{1} \rightleftharpoons e_{2}\right) e_{3} \Downarrow\left(a_{1} a_{2}\right) \cdot c}
\end{aligned}
$$

Fig. 3. $\lambda \nu$-PCF evaluation rules

Therefore the type of free atomic names is always name and we do not need to add a component to $\Gamma$ tracking the types of free atomic names in $e$.

Definition 6.2 ( $\lambda \nu$-PCF evaluation). In Figure 3 we extend PCF's usual rules for an inductively defined big-step evaluation relation with the rules below the dotted line that concern atomic names. Thus the relation $e \Downarrow c$ defines when a $\lambda \nu$-PCF expression $e$ evaluates to canonical form $c$. (See Figure 1 for the grammar of canonical forms.) As for PCF, we only evaluate expressions that are variable-closed in the sense that $\mathrm{fv}(e)=\emptyset$. However, expressions for evaluation may contain free atomic names; this is because, unlike variables, atomic names are canonical forms.

The $\lambda \nu$-PCF evaluation relation is easily seen to be deterministic $\left(e \Downarrow c \wedge e \Downarrow c^{\prime} \Rightarrow\right.$ $c=c^{\prime}$ ) and type-sound $(\emptyset \vdash e: \tau \wedge e \Downarrow c \Rightarrow \emptyset \vdash c: \tau)$. It is also equivariant:

$$
e \Downarrow c \Rightarrow \pi \cdot e \Downarrow \pi \cdot c .
$$

The evaluation rule in Figure 3 for local names makes use of the auxiliary definition in Figure 4, which implements the characteristic features of Odersky's functional theory of local names [Odersky 1994]: scopes intrude in a type-directed fashion. The operation of name restriction on canonical forms, $a, c \mapsto a \backslash \backslash$, is partial because $a \backslash \backslash a:=c$ holds for no $c$. Thus, unlike Pitts [2011], we choose to follow Odersky [1994] and make $\nu a$. $a$ a stuck expression that does not evaluate to any canonical form and whose denotation is $\perp$.

Remark 6.3 (generative names). The use of Odersky-style local names means that the operational semantics of $\lambda \nu$-PCF is stateless, unlike the operational semantics of the more usual, generative version of $\nu a$. - used in the $\nu$-calculus [Pitts and Stark 1993] and most practical languages. However, Odersky-style local names are known to be as expressive as generative ones, at least in a simply typed setting. This follows from the existence of an adequate continuation-passing style translation from $\nu$-calculus into the $\lambda \nu$-calculus [Lösch and Pitts 2011]. Indeed here we do not escape the subtle 


$$
\frac{c \in\{\mathrm{T}, \mathrm{F}, \mathrm{O}\}}{a \| c:=c} \quad \frac{a \| c:=c^{\prime}}{a \| \mathrm{S} c:=\mathrm{S} c^{\prime}} \quad \frac{a \neq a^{\prime}}{a \| a^{\prime}:=a^{\prime}} \quad \overline{a \|\left(e_{1}, e_{2}\right):=\left(\nu a \cdot e_{1}, \nu a \cdot e_{2}\right)}
$$

$$
\overline{a \Uparrow \lambda x: \tau \rightarrow e:=\lambda x: \tau \rightarrow \nu a . e}
$$

Fig. 4. $\lambda \nu$-PCF partial operation of name restriction

properties of generative names modulo contextual equivalence, but encounter them higher up the type hierarchy-see (27) in Example 6.5 below.

We adopt the usual definitions of PCF contextual preorder and contextual equivalence (observing the termination behaviour of expressions of higher type in contexts of ground type), arriving at the following definition for $\lambda \nu$-PCF:

Definition 6.4 (contextual equivalence). As usual, a $\lambda \nu$-PCF context $C$ [-] is an expression with a single sub-expression replaced by the place-holder ',', and $C[e]$ is the expression that results from replacing - by an expression $e$ (possibly capturing free variables and atomic names in $e$ ). Given well-typed expressions $\Gamma \vdash e: \tau$ and $\Gamma \vdash e^{\prime}: \tau$, we write $\Gamma \vdash e \leq_{\lambda \nu \text {-PCF }} e^{\prime}: \tau$ and say that $e$ and $e^{\prime}$ are in the contextual preorder if for all contexts $C$ []] for which $\emptyset \vdash C[e]$ : bool and $\emptyset \vdash C\left[e^{\prime}\right]$ : bool hold, it is the case that $C[e] \Downarrow \mathrm{T}$ implies $C\left[e^{\prime}\right] \Downarrow \mathrm{T}$. The equivalence relation generated by this preorder is written $\cong_{\lambda \nu \text { PCF }}$ and is called contextual equivalence.

Example 6.5. Although $\lambda \nu$-PCF contains the $\nu$-calculus [Pitts and Stark 1993] as a subset, the two languages have very different semantics for local names - Oderskystyle for $\lambda \nu$-PCF versus generative for the $\nu$-calculus. This affects properties of contextual equivalence in the two languages. For example, if $\Gamma, x: \tau \vdash e: \tau^{\prime}$, then

$$
\Gamma \vdash \nu a . \lambda x: \tau \rightarrow e \cong_{\lambda \nu \text { PCF }} \lambda x: \tau \rightarrow \nu a . e: \tau \rightarrow \tau^{\prime}
$$

is valid. Indeed, this is a 'Kleene equivalence' for $\lambda \nu$-PCF (one expression evaluates to a canonical form if and only if the other does and in that case the canonical forms are equal); and all such identities can be proved by checking that they hold in the denotational model developed in the next section and then appealing to the computational adequacy result proved there (Theorem 7.5). However, (16) does not always hold in the $\nu$-calculus [Pitts and Stark 1993, Example 2]. On the other hand, analogues of some $\nu$-calculus equivalences are also true for $\lambda \nu$-PCF, once one takes into account the fact that, like PCF, $\lambda \nu$-PCF is call-by-name, whereas the $\nu$-calculus is call-by-value. For example, here are call-by-name analogues of two equivalences in Pitts and Stark [1993, Example 4]:

$$
\begin{gathered}
\emptyset \vdash \nu a . \lambda x: \text { name } \rightarrow(x=a) \cong_{\lambda \nu \text { PCF }} \lambda x: \text { name } \rightarrow \text { if } x=x \text { then } \mathrm{F} \text { else } \mathrm{F}: \text { name } \rightarrow \text { bool } \\
\emptyset \vdash \nu a . \nu a^{\prime} \cdot \lambda(f: \text { name } \rightarrow \text { bool }) \rightarrow \text { eq }(f a)\left(f a^{\prime}\right) \cong_{\lambda \nu \text { PCF }} \\
\lambda(f: \text { name } \rightarrow \text { bool }) \rightarrow \nu a \text {. if } f a \text { then } \mathrm{T} \text { else } \mathrm{T}:(\text { name } \rightarrow \text { bool }) \rightarrow \text { bool }
\end{gathered}
$$

where eq : bool $\rightarrow$ bool $\rightarrow$ bool is an abbreviation for a boolean-equality test, defined using conditionals. In contrast to the $\nu$-calculus, where it takes some effort to prove equivalences like (17) and (18) (see Tzevelekos [2012]), for $\lambda \nu$-PCF these properties are easily seen to hold in the straightforward and computationally adequate denotational semantics that we describe in the next section; see Example 7.6. However, this is not always the case: there are valid instances of the $\lambda \nu$-PCF contextual preorder and 
equivalence that cannot be established by calculating denotations in our model. Here are two examples of this phenomenon, suggested by Tzevelekos [private communication]. Consider the following variable-closed $\lambda \nu$-PCF expressions:

$$
\begin{aligned}
& \text { bot }_{\tau} \triangleq \mathrm{fix}(\lambda x: \tau \rightarrow x) \\
& \text { eqBot }_{a} \triangleq \lambda x: \text { name } \rightarrow \text { if } x=a \text { then } \mathrm{T} \text { else bot } \text { bool } \\
& \mathrm{kBot} \triangleq \lambda x: \text { name } \rightarrow \text { bot }_{\mathrm{bool}}
\end{aligned}
$$

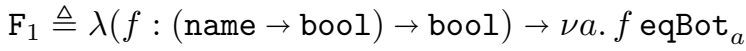

$$
\begin{aligned}
& \mathrm{F}_{2} \triangleq \lambda(f:(\text { name } \rightarrow \text { bool }) \rightarrow \text { bool }) \rightarrow f \text { kBot } \\
& \mathrm{eq}_{a} \triangleq \lambda x: \text { name } \rightarrow(x=a) \\
& \mathrm{G}_{1} \triangleq \lambda(g:(\text { name } \rightarrow \text { bool }) \rightarrow \text { name }) \rightarrow \nu a .\left(g \text { eq }_{a}=a\right) \\
& \mathrm{G}_{2} \triangleq \lambda(g:(\text { name } \rightarrow \text { bool }) \rightarrow \text { name }) \rightarrow \mathrm{F} .
\end{aligned}
$$

Thus eqBot ${ }_{a}, \mathrm{kBot}$ and $\mathrm{eq}_{a}$ are of type name $\rightarrow$ bool; $\mathrm{F}_{1}$ and $\mathrm{F}_{2}$ are of type $(($ name $\rightarrow$ bool $) \rightarrow$ bool $) \rightarrow$ bool; and $G_{1}$ and $G_{2}$ are of type $(($ name $\rightarrow$ bool $) \rightarrow$ name $) \rightarrow$ bool. We claim that

$$
\begin{aligned}
& \emptyset \vdash \mathrm{F}_{1} \cong_{\lambda \nu \text {-PCF }} \mathrm{F}_{2}:((\text { name } \rightarrow \text { bool }) \rightarrow \text { bool }) \rightarrow \text { bool } \\
& \emptyset \vdash \mathrm{G}_{1} \leq_{\lambda \nu \text {-PCF } \mathrm{G}_{2}:((\text { name } \rightarrow \text { bool }) \rightarrow \text { name }) \rightarrow \text { bool }} .
\end{aligned}
$$

The intuitive justification for (27) is that whatever argument of type (name $\rightarrow$ bool) $\rightarrow$ bool is supplied for $f$ by a context, it cannot have a free occurrence of $a$ (because substitution for $f$ in $\nu a .\left(f \mathrm{eq}_{a}\right)$ is capture-avoiding) and hence, it is claimed, cannot distinguish eq $\mathrm{q}_{a}$ from $\mathrm{k}_{\mathrm{F}}$. Similarly for (28), whatever argument of type (name $\rightarrow$ bool) $\rightarrow$ name is supplied for $g$ by a context will not contain $a$ free and hence, it is claimed, cannot produce $a$ when applied to $\mathrm{eq}_{a}$. (Since that application may diverge, in (28) we only have $\leq_{\lambda \nu \text {-PCF }}$ rather than $\cong_{\lambda \nu \text {-PCF. }}$. Formal proofs of this intuition are sketched in Appendix A.

Once we add existential quantification over names (Example 5.7) to the programming language, one does gain the ability to distinguish eq $q_{a}$ from $\mathrm{k}_{\mathrm{F}}$ with a test not involving $a$ free; and adding definite name description (Example 5.8) allows one to re-

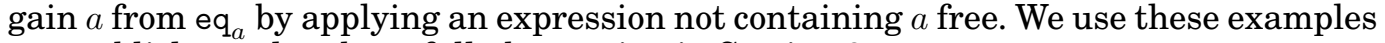
to establish results about full abstraction in Section 8.

\section{DENOTATIONAL SEMANTICS OF $\lambda \nu$-PCF}

For each $\lambda \nu$-PCF type $\tau$, we define a nominal Scott domain $\llbracket \tau \rrbracket$ by recursion on the structure of $\tau$ as follows:

- $\llbracket$ bool $\rrbracket=2_{\perp}$, the flat domain (cf. Definition 5.2) on a discrete nominal set with two elements, $2=\{$ true, false $\}$.

- \nat $\rrbracket=\mathbb{N}_{\perp}$, the flat domain on the discrete nominal set of natural numbers, $\mathbb{N}=\{0,1,2, \ldots\}$.

- $\llbracket \tau \times \tau^{\prime} \rrbracket=\llbracket \tau \rrbracket \times \llbracket \tau^{\prime} \rrbracket$, the product of nominal Scott domains.

- $\llbracket \tau \rightarrow \tau^{\prime} \rrbracket=\llbracket \tau \rrbracket \rightarrow \llbracket \tau^{\prime} \rrbracket$, the nominal Scott domain of uniform-continuous functions (the exponential in the cartesian closed category Nsd, Theorem 5.5).

- $\llbracket$ name $\rrbracket=\mathbb{A}_{\perp}$, the flat domain on the nominal set of atomic names, $\mathbb{A}=\{a, b, c, \ldots\}$.

Typing environments are interpreted as finite cartesian products:

$$
\llbracket\left\{x_{1}: \tau_{1}, \ldots, x_{n}: \tau_{n}\right\} \rrbracket=\llbracket \tau_{1} \rrbracket \times \cdots \times \llbracket \tau_{n} \rrbracket .
$$

Finite tuples $\rho \in \llbracket \Gamma \rrbracket$ can be interpreted as partial functions from variables to domains such that $\operatorname{dom}(\rho)=\operatorname{dom}(\Gamma)$ and $\rho(x) \in \llbracket \Gamma(x) \rrbracket$ for all $x \in \operatorname{dom}(\Gamma)$. We call such partial 


$$
\begin{aligned}
& \llbracket x \rrbracket \rho=\rho x \quad \llbracket \mathrm{T} \rrbracket \rho=\text { true } \quad \llbracket \mathrm{F} \rrbracket \rho=\text { false } \quad \llbracket \mathrm{O} \rrbracket \rho=0 \\
& \llbracket \text { if } e_{1} \text { then } e_{2} \text { else } e_{3} \rrbracket \rho=\left\{\begin{array}{ll}
\llbracket e_{2} \rrbracket \rho & \text { if } \llbracket e_{1} \rrbracket \rho=\text { true } \\
\llbracket e_{3} \rrbracket \rho & \text { if } \llbracket e_{1} \rrbracket \rho=\text { false } \\
\perp & \text { otherwise }
\end{array} \quad \llbracket \mathrm{S} e \rrbracket \rho= \begin{cases}n+1 & \text { if } \llbracket e \rrbracket \rho=n \\
\perp & \text { otherwise }\end{cases} \right. \\
& \llbracket \text { pred } e \rrbracket \rho=\left\{\begin{array}{ll}
n & \text { if } \llbracket e \rrbracket \rho=n+1 \\
\perp & \text { otherwise }
\end{array} \quad \llbracket \text { zero } e \rrbracket \rho= \begin{cases}\text { true } & \text { if } \llbracket e \rrbracket \rho=0 \\
\text { false } & \text { if } \llbracket e \rrbracket \rho=n+1 \\
\perp & \text { otherwise }\end{cases} \right. \\
& \llbracket\left(e_{1}, e_{2}\right) \rrbracket \rho=\left(\llbracket e_{1} \rrbracket \rho, \llbracket e_{2} \rrbracket \rho\right) \quad \llbracket \text { fst } e \rrbracket \rho=\pi_{1}(\llbracket e \rrbracket \rho) \quad \llbracket \text { snd } e \rrbracket \rho=\pi_{2}(\llbracket e \rrbracket \rho) \\
& \llbracket \lambda x: \tau \rightarrow e \rrbracket \rho=\lambda d \in \llbracket \tau \rrbracket \rightarrow \llbracket e \rrbracket \rho[x \mapsto d] \quad \llbracket e_{1} e_{2} \rrbracket \rho=\llbracket e_{1} \rrbracket \rho\left(\llbracket e_{2} \rrbracket \rho\right) \quad \llbracket \text { fix } e \rrbracket \rho=\operatorname{fix}(\llbracket e \rrbracket \rho) \\
& \llbracket a \rrbracket \rho=a \quad \llbracket \nu a . e \rrbracket \rho=(a \backslash \llbracket e \rrbracket) \rho \quad \llbracket e_{1}=e_{2} \rrbracket \rho= \begin{cases}\text { true } & \text { if } \llbracket e_{i} \rrbracket \rho=a_{i} \text { and } a_{1}=a_{2} \\
\text { false } & \text { if } \llbracket e_{i} \rrbracket \rho=a_{i} \text { and } a_{1} \neq a_{2} \\
\perp & \text { otherwise }\end{cases} \\
& \llbracket\left(e_{1} \rightleftharpoons e_{2}\right) e_{3} \rrbracket \rho= \begin{cases}\left(a_{1} a_{2}\right) \cdot\left(\llbracket e_{3} \rrbracket \rho\right) & \text { if } \llbracket e_{i} \rrbracket \rho=a_{i} \\
\perp & \text { otherwise }\end{cases}
\end{aligned}
$$

Fig. 5. $\lambda \nu$-PCF denotational semantics

functions $\Gamma$-valuations. If $\rho \in \llbracket \Gamma \rrbracket, x \notin \operatorname{dom}(\Gamma)$ and $d \in \llbracket \tau \rrbracket$, then we write $\rho[x \mapsto d]$ for the $(\Gamma, x: \tau)$-valuation that maps $x$ to $d$ and otherwise acts like $\rho$.

The denotation of each well-typed expression $\Gamma \vdash e: \tau$ is an element

$$
\llbracket e \rrbracket \in \llbracket \Gamma \rrbracket \rightarrow \llbracket \tau \rrbracket
$$

of the exponential domain $\llbracket \Gamma \rrbracket \rightarrow \llbracket \tau \rrbracket$; in other words, $\llbracket e \rrbracket$ is a uniform-continuous function mapping $\Gamma$-valuations $\rho \in \llbracket \Gamma \rrbracket$ to elements $\llbracket e \rrbracket \rho$ of the nominal Scott domain $\llbracket \tau \rrbracket$. Figure 5 gives the definition of these functions, by recursion over the structure ${ }^{4}$ of $e$. The denotations of constructs from PCF are analogous to the standard denotational semantics of PCF in Scott domains. The functions $\pi_{1}$ and $\pi_{2}$ in the clauses for fst $e$ and snd $e$ are the first and second projection functions for pairs; and fix in the clause for $\mathrm{fix} e$ is the least fixed point function (14). The clauses below the dotted line in Figure 5 are for the syntactic constructs that $\lambda \nu$-PCF adds to PCF. Atomic names are their own denotation. The semantics of explicit swapping expressions, $\left(e_{1} \rightleftharpoons e_{2}\right) e_{3}$, make use of the action of finite permutations that each nominal Scott domain possesses. Finally, to interpret expressions with locally scoped names, $\nu$ a.e, we use uniform-continuous functions

$$
a \backslash(-) \in(\llbracket \Gamma \rrbracket \rightarrow \llbracket \tau \rrbracket) \rightarrow(\llbracket \Gamma \rrbracket \rightarrow \llbracket \tau \rrbracket)
$$

that are name restriction operations [Pitts 2013, Definition 9.2]:

Definition 7.1 (uniform-continuous name restriction). A uniform-continuous name restriction operation on a nominal Scott domain $D$ is a morphism (-) $\backslash\left({ }_{-}\right): \mathbb{A}_{\perp} \times D \rightarrow D$

\footnotetext{
${ }^{4}$ Strictly speaking, it is by $\alpha$-structural recursion [Pitts 2006], since we identify expressions up to $\alpha$ equivalence of bound identifiers.
} 
in Nsd satisfying for all $a, a^{\prime} \in \mathbb{A}$ and $d \in D$

$$
\begin{gathered}
\perp \backslash d=\perp \\
a \#(a \backslash d) \\
a \# d \Rightarrow a \backslash d=d \\
a \backslash\left(a^{\prime} \backslash d\right)=a^{\prime} \backslash(a \backslash d)
\end{gathered}
$$

(where as usual _ \# _ is the freshness relation (5) for $D$ ). Since $\left(_{-}\right) \backslash\left(_{-}\right)$is equivariant, (30) is equivalent to asking that $a \backslash\left(_{-}\right)$be a binding operation, that is, respect the generalized version of $\alpha$-equivalence that any nominal set supports: $a^{\prime} \#(a, d) \Rightarrow a \backslash d=$ $a^{\prime} \backslash\left(\left(a a^{\prime}\right) \cdot d\right) .(31)$ and $(32)$ are basic structural properties that one expects any notion of local scoping to have; see the discussion at the start of Section 9.1 of Pitts [2013].

THEOREM 7.2. Every flat nominal Scott domain $X_{\perp}$ has a uniform-continuous name restriction operation satisfying

$$
a \backslash d= \begin{cases}d & \text { if } a \# d \\ \perp & \text { if } a \in \operatorname{supp} d\end{cases}
$$

for all $a \in \mathbb{A}$ and $d \in X_{\perp}$. If $D_{1}, D_{2} \in \mathrm{Nsd}$ have uniform-continuous name restriction operations, their product $D_{1} \times D_{2}$ has one satisfying

$$
a \backslash\left(d_{1}, d_{2}\right)=\left(a \backslash d_{1}, a \backslash d_{2}\right)
$$

for all $a \in \mathbb{A}, d_{1} \in D_{1}$ and $d_{2} \in D_{2}$. If $D_{1}, D_{2} \in \mathrm{Nsd}$ and $D_{2}$ has a uniform-continuous name restriction operation, then whether or not $D_{1}$ has one as well, the exponential $D_{1} \rightarrow D_{2}$ has such an operation, satisfying

$$
(a \backslash f) d=a \backslash(f d) \quad \text { if } a \# d
$$

for all $d \in D_{1}$ and $f \in D_{1} \rightarrow D_{2}$.

PRoof. It is easy to see that (33) and (34) determine uniform-continuous name restriction operations on flat and product domains respectively. The case for exponential domains is less straightforward. (35) at first seems like only a partial specification for the function $a \backslash f: D_{1} \rightarrow D_{2}$, but in fact determines it uniquely, since it implies that for all $d \in D_{1}, f \in D_{1} \rightarrow D_{2}$ and $a \in \mathbb{A}$

$$
(a \backslash f) d=a^{\prime} \backslash\left(\left(\left(a a^{\prime}\right) \cdot f\right) d\right) \quad \text { for some, or indeed any } a^{\prime} \#(f, d) .
$$

This and (29)-(32) follow from the results for nominal sets in Pitts [2013, Section 9.3]. One also has to check that each function $a \backslash f$ is uniform-continuous and that $f \mapsto a \backslash f$ is uniform-continuous, but these are routine calculations.

Using this theorem we get a uniform-continuous name restriction operation on the denotation $\llbracket \tau \rrbracket$ of each $\lambda \nu$-PCF type $\tau$, by recursion on the structure of $\tau$; and then we use the theorem again to lift the name restriction operation on $\llbracket \tau \rrbracket$ to one on the exponential domain $\llbracket \Gamma \rrbracket \rightarrow \llbracket \tau \rrbracket$, for each typing environment $\Gamma$. It is this operation which is used in Figure 5 to give the denotation of locally scoped expressions $\nu a$.e. From (35) we have

$$
\llbracket \nu a . e \rrbracket \rho=a \backslash(\llbracket e \rrbracket \rho) \quad \text { if } a \# \rho
$$

where the name restriction operation on the right-hand side is that for $\llbracket \tau \rrbracket$. Note that the side condition $a \# \rho$ is always satisfiable, since we identify expressions up to $\alpha$ equivalence of $\nu$-bound atomic names. 
Notation 7.3. For the empty typing environment $\emptyset$, $\llbracket \emptyset \rrbracket=1$ contains a unique $\emptyset$ valuation, $\rho_{0}$. Given a variable-closed expression $\emptyset \vdash e: \tau$, we simply write $e \rrbracket$ for $\llbracket e \rrbracket \rho_{0}$.

LEMMA 7.4 ( $\lambda \nu$-PCF SOUNDNESS). If $e$ is a well-typed, variable-closed $\lambda \nu$-PCF expression and $e \Downarrow c$, then $\llbracket e \rrbracket=\llbracket c \rrbracket$.

PROOF. The proof is by induction on the derivation of $e \Downarrow c$ from the rules in Figure 3 and most of the induction steps are routine. As for PCF, to establish closure under the rule for evaluating function applications we need the following substitution property, which can be proved by induction on the structure of expressions:

Substitution Lemma. If $\Gamma \vdash e: \tau$ and $\Gamma, x: \tau \vdash e^{\prime}: \tau^{\prime}$, then $\Gamma \vdash e^{\prime}[e / x]: \tau^{\prime}$ holds

and for all $\rho \in \llbracket \Gamma \rrbracket$ we have $\llbracket e^{\prime}[e / x] \rrbracket \rho=\llbracket e^{\prime} \rrbracket \rho[x \mapsto \llbracket e \rrbracket \rho]$.

For closure under the rule for evaluating explicit swapping expressions, we use the fact that the denotational semantics is equivariant:

Equivariance Lemma. For all $\Gamma \vdash e: \tau$ and all finite permutations $\pi$ it holds that $\pi \cdot \llbracket e \rrbracket=\llbracket \pi \cdot e \rrbracket$.

Thus we also have $\left(a_{1} a_{2}\right) \cdot \llbracket c \rrbracket=\llbracket\left(a_{1} a_{2}\right) \cdot c \rrbracket$ for all variable-closed canonical forms $c$. Finally, for closure under the rule for evaluating locally scoped expressions, we need the following property of the name restriction operations $a \backslash(-) \in \llbracket \tau \rrbracket \rightarrow \llbracket \tau \rrbracket$ :

Restriction Lemma. If $c$ and $c^{\prime}$ are variable-closed canonical forms of type $\tau$ satisfying $a \rrbracket c:=c^{\prime}$ (see Figure 4), then $a \backslash \llbracket c \rrbracket=\llbracket c^{\prime} \rrbracket$ in $\llbracket \tau \rrbracket$.

This follows from the definition in Figure 4, by induction on the structure of $c$; for $\lambda$ abstractions one needs the fact, noted in the proof of Theorem 7.2, that (35) uniquely determines the name restriction operation on functions defined by (36).

The following result allows one to establish $\lambda \nu$-PCF contextual equivalences by proving equality of denotations.

THEOREM 7.5 ( $\lambda \nu$-PCF COMPUTATIONAL ADEQUACY). For any $\Gamma \vdash e: \tau$ and $\Gamma \vdash$ $e^{\prime}: \tau$, if $\llbracket e \rrbracket \sqsubseteq \llbracket e^{\prime} \rrbracket \in \llbracket \Gamma \rrbracket \rightarrow \llbracket \tau \rrbracket$, then $\Gamma \vdash e \leq_{\lambda \nu \text {-PCF }} e^{\prime}: \tau$. Consequently, if $\llbracket e \rrbracket=\llbracket e^{\prime} \rrbracket$, then $\Gamma \vdash e \cong_{\lambda \nu}$ PCF $e^{\prime}: \tau$.

Proof. It is evident from Figure 5 that the denotational semantics is compositional, in the sense that for any suitably typed context $C[-]$ one has

$$
\llbracket e \rrbracket \sqsubseteq \llbracket e^{\prime} \rrbracket \Rightarrow \llbracket C[e] \rrbracket \sqsubseteq \llbracket C\left[e^{\prime}\right] \rrbracket .
$$

So in view of Lemma 7.4 it suffices to show that if $e$ is a variable-closed expression of type bool, then

$$
\llbracket e \rrbracket=\text { true } \Rightarrow e \Downarrow \mathrm{T} .
$$

As for PCF, this can be proved by defining a suitable logical relation

$$
d \triangleleft_{\tau} e \quad(d \in \llbracket \tau \rrbracket, \emptyset \vdash e: \tau)
$$

between the semantics and the syntax of $\lambda \nu$-PCF. See Streicher [2006, Chapter 4] for a good exposition of this method of proving computational adequacy for the Scott domain model of PCF. We outline the method, concentrating on how the move to nominal Scott domains allows us to deal with the new features of $\lambda \nu$-PCF. 
The definition of (42) is by recursion on the structure of the type $\tau$ :

$$
\begin{aligned}
d \triangleleft_{\gamma} e & \triangleq d=\perp \vee(\exists c) e \Downarrow c \wedge \llbracket c \rrbracket=d \quad \text { for } \gamma \in\{\text { bool, nat, name }\} \\
\left(d_{1}, d_{2}\right) \triangleleft_{\tau_{1} \times \tau_{2}} e & \triangleq d_{1} \triangleleft_{\tau_{1}} \text { fst } e \wedge d_{2} \triangleleft_{\tau_{2}} \text { snd } e \\
d \triangleleft_{\tau_{1} \rightarrow \tau_{2}} e & \triangleq\left(\forall d_{1}, e_{1}\right) d_{1} \triangleleft_{\tau_{1}} e_{1} \Rightarrow d d_{1} \triangleleft_{\tau_{2}} e e_{1} .
\end{aligned}
$$

The relation is extended to typing environments $\Gamma$ by:

$$
\rho \triangleleft_{\Gamma} s \triangleq(\forall x \in \operatorname{dom}(\Gamma)) \rho x \triangleleft_{\Gamma x} s x .
$$

Here $\rho \in \llbracket \Gamma \rrbracket$ and $s$ ranges over $\Gamma$-substitutions, which by definition are functions mapping each variable $x \in \operatorname{dom}(\Gamma)$ to a variable-closed expression $s x$ of type $\Gamma x$. This extension allows us to state the

Fundamental Property of the Logical Relation. For every $\Gamma \vdash e: \tau, \rho \in \llbracket \Gamma \rrbracket$ and $\Gamma$-substitution $s$

$$
\rho \triangleleft_{\Gamma} s \Rightarrow \llbracket e \rrbracket \rho \triangleleft_{\tau} e s
$$

where $e s$ is the variable-closed expression of type $\tau$ obtained by simultaneous substitution; thus $e s \triangleq e\left[e_{1} / x_{1}, \ldots, e_{n} / x_{n}\right]$ if $\operatorname{dom}(\Gamma)$ consists of the distinct variables $x_{1}, \ldots, x_{n}$ and $s x_{i}=e_{i}$ for $i=1, \ldots, n$.

The fundamental property (44) is proved by induction on the derivation of $\Gamma \vdash e: \tau$ from the rules in Figure 2. As for PCF, the proof makes use of the 'Kleene preorder' defined by

$$
e \leq^{k} e^{\prime} \triangleq(\forall c) e \Downarrow c \Rightarrow e^{\prime} \Downarrow c
$$

together with the fact, established by induction on the structure of $\tau$, that the logical relation is closed under this preorder:

$$
d \triangleleft_{\tau} e \wedge e \leq^{k} e^{\prime} \Rightarrow d \triangleleft_{\tau} e^{\prime} .
$$

The induction steps in the proof of (44) for PCF constructs are standard, except that when it comes to fixed point recursion one uses the facts (easily verified by induction on $\tau$ ) that each $\triangleleft_{\tau} e$ satisfies $\perp \triangleleft_{\tau} e$ and $\left((\forall d \in S) d \triangleleft_{\tau} e\right) \Rightarrow \bigsqcup S \triangleleft_{\tau} e$ for all uniformdirected subsets $S \subseteq \llbracket \tau \rrbracket$. The induction step for name swapping $\left(e_{1} \rightleftharpoons e_{2}\right) e_{3}$ depends on the equivariance of the operational semantics (15), of the denotational semantics (39), and also of the logical relation

$$
d \triangleleft_{\tau} e \Rightarrow \pi \cdot d \triangleleft_{\tau} \pi \cdot e
$$

which can be proved by induction on $\tau$. The induction step for locally scoped names $\nu$ a.e follows directly from the fact that the logical relation is closed under name restriction:

$$
d \triangleleft_{\tau} e \Rightarrow a \backslash d \triangleleft_{\tau} \nu a . e .
$$

This in turn can be shown by induction on $\tau$, where we use some easily checked facts about the Kleene preorder:

$$
\begin{aligned}
& \nu a . \text { fst } e \leq^{k} \text { fst } \nu a . e \\
& \nu a \text {. snd } e \leq{ }^{k} \text { snd } \nu a . e \\
& a \# e_{1} \Rightarrow \nu a .\left(e e_{1}\right) \leq^{k}(\nu a . e) e_{1}
\end{aligned}
$$

together with (46) and the fact that $\nu a ._{-}$is a binder.

As a corollary of the fundamental property (44) we have that every variable-closed expression $e$ of type bool satisfies $\llbracket e \rrbracket \triangleleft_{\text {bool }} e$. Consequently, if $\llbracket e \rrbracket=$ true, then by definition of $\triangleleft_{\text {bool }}$ we must have $e \Downarrow c$ for some $c$ with $\llbracket c \rrbracket=$ true, that is, $e \Downarrow \mathrm{T}$. So we do indeed have (41) and this completes the proof of the theorem. 


$$
\begin{aligned}
& e \in \operatorname{Exp}::= \\
& \vdots \\
& \text { ex } x . e \\
& \text { the } x . e \\
& \text { expressions } \\
& \text { (as for } \lambda \nu \text {-PCF) } \\
& \text { existential name quantification } \\
& \text { definite name description } \\
& \frac{\Gamma, x: \text { name } \vdash e: \text { bool }}{\Gamma \vdash \text { ex } x . e: \text { bool }} \quad \frac{\Gamma, x: \text { name } \vdash e: \text { bool }}{\Gamma \vdash \text { the } x . e: \text { name }} \\
& \frac{a \in \mathbb{A} \quad e[a / x] \Downarrow \mathrm{T}}{\operatorname{exx} . e \Downarrow \mathrm{T}} \quad \frac{b \# e \quad\left(\forall a^{\prime} \in \mathrm{fn}(e) \cup\{b\}\right) e\left[a^{\prime} / x\right] \Downarrow \mathrm{F}}{\operatorname{exx} . e \Downarrow \mathrm{F}} \\
& \frac{e[a / x] \Downarrow \mathrm{T} \quad b \#(e, a) \quad\left(\forall a^{\prime} \in(\mathrm{fn}(e)-\{a\}) \cup\{b\}\right) e\left[a^{\prime} / x\right] \Downarrow \mathrm{F}}{\text { the } x . e \Downarrow a} \\
& \llbracket \operatorname{ex} x . e \rrbracket \rho=\operatorname{exists}_{\mathbb{A}}(\llbracket \lambda x: \text { name } \rightarrow e \rrbracket \rho) \quad \llbracket \text { the } x . e \rrbracket \rho=\text { the }_{\mathbb{A}}(\llbracket \lambda x: \text { name } \rightarrow e \rrbracket \rho)
\end{aligned}
$$

Fig. 6. Syntax and semantics of $\lambda \nu-\mathrm{PCF}^{+}$

Example 7.6. Using Theorem 7.5 one can prove many contextual equivalences in $\lambda \nu$-PCF, such as (16)-(18), in a straightforward manner via the denotational semantics. We prove (17) in detail. Since we identify expressions up to $\alpha$-equivalence, for any given $a^{\prime} \in \mathbb{A}$ we can pick a representative expression $\nu a . \lambda x:$ name $\rightarrow(x=a)$ such that $a \neq a^{\prime}$, then

$$
\begin{aligned}
& \llbracket \nu a . \lambda x: \text { name } \rightarrow(x=a) \rrbracket a^{\prime} \\
& =(a \backslash \llbracket \lambda x: \text { name } \rightarrow(x=a) \rrbracket) a^{\prime} \\
& =a \backslash\left(\llbracket \lambda x: \text { name } \rightarrow(x=a) \rrbracket a^{\prime}\right) \\
& =a \backslash \text { false } \\
& =\text { false } \\
& =\llbracket \lambda x: \text { name } \rightarrow \text { if } x=x \text { then } \mathrm{F} \text { else } \mathrm{F} \rrbracket a^{\prime} .
\end{aligned}
$$

using the definition in Figure 5

by (35), since $a \neq a^{\prime}$

as $a \neq a^{\prime}$

by (33)

Similarly $\llbracket \nu a . \lambda x:$ name $\rightarrow(x=a) \rrbracket \perp=\perp=\llbracket \lambda x:$ name $\rightarrow$ if $x=x$ then $\mathrm{F}$ else $\mathrm{F} \rrbracket \perp$. Hence $\llbracket \nu a . \lambda x:$ name $\rightarrow(x=a) \rrbracket=\llbracket \lambda x:$ name $\rightarrow$ if $x=x$ then $\mathrm{F}$ else $\mathrm{F} \rrbracket$ and so (17) holds by Theorem 7.5.

To prove (18) one can combine the definition in Figure 5 with the fact that if $a, a^{\prime} \#$ $f \in\left(\mathbb{A}_{\perp} \rightarrow 2_{\perp}\right) \rightarrow 2_{\perp}$, then $f a=f\left(\left(a a^{\prime}\right) \cdot a^{\prime}\right)=\left(a a^{\prime}\right) \cdot\left(f a^{\prime}\right)=f a^{\prime}$ (since $\left.f a^{\prime} \in 2_{\perp}\right)$.

\section{FULL ABSTRACTION}

Plotkin [1977] famously proves that the Scott domain model of PCF is computationally adequate, but not fully abstract: equality of denotations implies, but is not implied by, PCF contextual equivalence. Furthermore, he shows that the Scott model becomes fully abstract once one extends PCF with a parallel-or construct.

A similar story unfolds for $\lambda \nu$-PCF. We prove in Theorem 8.5 that the nominal Scott domain model becomes fully abstract once we add the ex $x . e$ and the $x$.e constructs from Figure 6 to $\lambda \nu-\mathrm{PCF}^{+}$. If we leave one of these constructs out, then full abstraction fails, as Theorem 8.4 shows.

Definition 8.1. The language $\lambda \nu-\mathrm{PCF}^{+}$is obtained by extending $\lambda \nu$-PCF with expressions for existentially quantifying over name ('there exists some $x$ : name such 
that...') and for forming definite descriptions over name ('the unique $x$ : name such that...'). The syntax, typing, evaluation rules and denotational semantics for this extension are given in Figure 6, where the functions exists $\mathbb{A}_{\mathbb{A}} \in \mathrm{K}\left(\left(\mathbb{A}_{\perp} \rightarrow 2_{\perp}\right) \rightarrow 2_{\perp}\right)$ and the $e_{\mathbb{A}} \in \mathrm{K}\left(\left(\mathbb{A}_{\perp} \rightarrow 2_{\perp}\right) \rightarrow \mathbb{A}_{\perp}\right)$ are defined in Examples 5.7 and 5.8. Both ex x.e and the $x$.e bind the variable $x$ in $e$. Contextual equivalence for the extended language $\Gamma \vdash e \cong_{\lambda \nu-\mathrm{PCF}^{+}} e^{\prime}: \tau$ is defined in the same way as it is for $\lambda \nu$-PCF in Definition 6.4.

Similarly we define $\lambda \nu$-PCF + ex to be $\lambda \nu$-PCF with only existential quantification added, and define $\lambda \nu$-PCF + the to be $\lambda \nu$-PCF with only definite description added.

Remark 8.2. The addition of existential quantification and definite description is mainly motivated by the need for them in our proof of full abstraction (Theorem 8.5). A less strict form of existential quantification for numbers (rather than, as here, for names) occurs in Plotkin's original PCF paper [Plotkin 1977]. Definite description has a long history in logic, but it is harder to motivate from a programming language perspective. Note that the analogue for numbers of our definite description and existential quantification functionals are not computable, as indicated already in Remark 5.9. The computability of ex $x$. $e$ and the $x$. e provide an example of the phenomenon of 'finite modulo symmetry' mentioned in the introduction. For example, to prove ex $x$. $e \Downarrow \mathrm{F}$, we just have to show $e\left[a^{\prime} / x\right] \Downarrow \mathrm{F}$ for each of the finitely many atomic names $a^{\prime}$ that occur free in $e$ and then pick any one of the infinitely many atomic names $b$ that do not occur free in $e$ and show $e[b / x] \Downarrow \mathrm{F}$; equivariance of evaluation (15) ensures that if $e[b / x] \Downarrow \mathrm{F}$, then $e\left[b^{\prime} / x\right] \Downarrow \mathrm{F}$ holds for any other $b^{\prime}$ not occurring free in $e$.

Remark 8.3 (parallel-or). The parallel-or construct is central in Plotkin's classical work on PCF [Plotkin 1977, Table 1], because PCF fails to be fully abstract without it, but becomes fully abstract with it. It is a boolean-valued operation that evaluates two boolean expressions 'in parallel' and is true whenever one of the expressions evaluates to true, no matter if the other one diverges. In the presence of ex $x . e$ we can define parallel-or as syntactic sugar by

$$
e \text { por } e^{\prime} \triangleq \nu a . \nu a^{\prime} \text {.ex } x \text {. if } x=a \text { then } e \text { else if } x=a^{\prime} \text { then } e^{\prime} \text { else } \mathrm{F}
$$

where $a, a^{\prime}$ and $x$ do not occur free in $e$ or $e^{\prime}$.

THEOREM 8.4 (FAILURE OF FULL ABSTRACTION). The denotational semantics of neither $\lambda \nu$-PCF+ex, nor $\lambda \nu$-PCF+the is fully abstract, in the sense that there are expressions in the contextual preorder relations of those languages which are not in the denotational partial order.

Proof. We start with $\lambda \nu$-PCF+the. Consider the two expressions $F_{1}$ and $F_{2}$ defined in (22) and (23). Their denotations are (equivariant and uniform-continuous) functions from $\left(\mathbb{A}_{\perp} \rightarrow 2_{\perp}\right) \rightarrow 2_{\perp}$ to $2_{\perp}$. Applying exists $_{\mathbb{A}}$ from Example 5.7 to them, we get

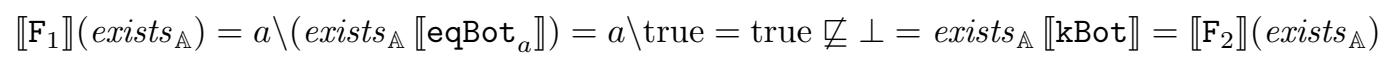

where eqBot ${ }_{a}$ and kBot are defined in (20) and (21). This shows that $\llbracket F_{1} \rrbracket \nsubseteq \llbracket F_{2} \rrbracket$. Nevertheless, in Appendix A we sketch the proof that

$$
\emptyset \vdash \mathrm{F}_{1} \leq_{\lambda \nu \text {-PCF }+ \text { the }} \mathrm{F}_{2}:((\text { name } \rightarrow \text { bool }) \rightarrow \text { bool }) \rightarrow \text { bool }
$$

which shows that $F_{1}$ and $F_{2}$ are counter examples to full abstraction for $\lambda \nu-P C F+$ the. We explained informally why (49) holds for $\lambda \nu$-PCF in Example 6.5; and adding the x.e does not add any ability to distinguish the evaluation behaviour of the two expressions in any boolean context. The formal proof of (49) in Appendix A works via an extensionality result (Proposition A.3) that is of interest in its own right. 
For $\lambda \nu$-PCF+ex, recall the expressions $G_{1}$ and $G_{2}$ from (25) and (26). They are in the contextual preorder for $\lambda \nu$-PCF+ex

$$
\emptyset \vdash \mathrm{G}_{1} \leq_{\lambda \nu-\mathrm{PCF}+\text { ex }} \mathrm{G}_{2}:((\text { name } \rightarrow \text { bool }) \rightarrow \text { name }) \rightarrow \text { bool } .
$$

and the proof sketch of this is in Appendix A. This provides a counter example to full abstraction for $\lambda \nu-\mathrm{PCF}+\mathrm{ex}$, because through applying the $e_{\mathbb{A}}$ from Example 5.8

$$
\llbracket \mathrm{G}_{1} \rrbracket\left(\text { the }_{\mathbb{A}}\right)=a \backslash\left(e q_{a}\left(\text { the }_{\mathbb{A}} \llbracket e \mathrm{q}_{a} \rrbracket\right)\right)=a \backslash\left(e q_{a} a\right)=a \backslash \text { true }=\text { true } \nsubseteq \text { false }=\llbracket \mathrm{G}_{2} \rrbracket\left(\text { the }_{\mathbb{A}}\right)
$$

(using the function $e q_{a}$ from (10) and the expression $e q_{a}$ from (24)), we obtain that $\llbracket \mathrm{G}_{1} \rrbracket \nsubseteq \llbracket \mathrm{G}_{2} \rrbracket$.

More positively, we now show that $\lambda \nu-\mathrm{PCF}^{+}$is fully abstract with respect to the nominal Scott domain model.

THEOREM 8.5 (FULl ABSTRACTION FOR $\lambda \nu-\mathrm{PCF}^{+}$). For all well-typed expressions $\Gamma \vdash e: \tau$ and $\Gamma \vdash e^{\prime}: \tau$ in $\lambda \nu-\mathrm{PCF}^{+}$, we have

$$
\llbracket e \rrbracket \sqsubseteq \llbracket e^{\prime} \rrbracket \in \llbracket \Gamma \rrbracket \rightarrow \llbracket \tau \rrbracket \Leftrightarrow \Gamma \vdash e \leq_{\lambda \nu-\mathrm{PCF}^{+}} e^{\prime}: \tau .
$$

and consequently $\llbracket e \rrbracket=\llbracket e^{\prime} \rrbracket \in \llbracket \Gamma \rrbracket \rightarrow \llbracket \tau \rrbracket \Leftrightarrow \Gamma \vdash e \cong_{\lambda \nu-\mathrm{PCF}^{+}} e^{\prime}: \tau$ holds.

The outline of the proof of this theorem occupies the rest of this section, with some technical details relegated to the appendices. First note that the extension of Theorem 7.5 to $\lambda \nu-\mathrm{PCF}^{+}$is straightforward and gives us the left-to-right implication in $\left(\mathrm{FA}_{\tau}\right)$. Establishing the reverse implication leads to an investigation of the uniform-compact elements (Definition 3.6) of nominal Scott domains of the form $\llbracket \tau \rrbracket$ for some type $\tau \in$ Typ.

Definition 8.6 (definability). An element in the nominal Scott domain model $d \in \llbracket \tau \rrbracket$ is $\lambda \nu$ - $\mathrm{PCF}^{+}$-definable if there is a variable-closed $\lambda \nu$-PCF ${ }^{+}$expression $\emptyset \vdash e: \tau$ that denotes it: $d=\llbracket e \rrbracket$. We say uniform-compact definability holds at a type $\tau$ if all uniformcompact elements of $\llbracket \tau \rrbracket$ are definable:

$$
(\forall u \in \mathrm{K} \llbracket \tau \rrbracket)(\exists e) \emptyset \vdash e: \tau \wedge u=\llbracket e \rrbracket .
$$

$\left(\mathrm{DEF}_{\tau}\right)$

As Curien [2007] surveys, knowing $\left(\mathrm{DEF}_{\tau}\right)$ for all types $\tau$ is enough to show the right-to-left implication in $\left(\mathrm{FA}_{\tau}\right)$. Our proof follows this proof pattern. However, we only show uniform-compact definability at certain types that avoid the use of function types $\tau_{1} \rightarrow \tau_{2}$ in which the nominal Scott domain $\llbracket \tau_{2} \rrbracket$ might contain elements with nonempty support. So $\tau_{2}=$ nat is OK, but $\tau_{2}=$ name is not, for example. This leads us to make the following definition:

Definition 8.7 (simple types). Let Styp $\subseteq$ Typ be the subset of types (Figure 1) given by the following grammar

$$
\sigma \in \text { Styp }::=\text { nat } \mid \text { name }|\sigma \times \sigma| \sigma \rightarrow \text { nat }
$$

and call types of that grammar simple types.

Note that $\sigma \rightarrow$ name is not in Styp. The following lemma is the key to the usefulness of simple types. It is where the presence of 'the $x . e$ ' expressions in $\lambda \nu-\mathrm{PCF}^{+}$gets used.

LEMMA 8.8. For any type $\tau \in$ Typ, there is a simple type $\sigma \in$ Styp such that there is a $\lambda \nu$ - $\mathrm{PCF}^{+}$-definable embedding-projection pair from $\tau$ to $\sigma$, meaning that there are closed $\lambda \nu$ - $\mathrm{PCF}^{+}$expressions $\emptyset \vdash e: \tau \rightarrow \sigma$ and $\emptyset \vdash p: \sigma \rightarrow \tau$ with $\llbracket \lambda x: \tau \rightarrow p(e x) \rrbracket=i d_{\llbracket \tau \rrbracket}$ and $\llbracket \lambda x: \sigma \rightarrow e(p x) \rrbracket \sqsubseteq i d_{\llbracket \sigma \rrbracket}$. 
PROOF. It is standard that identity functions are embedding-projections, that embedding-projections compose, and that products and functions give covariant functors with respect to embedding-projections; and all these constructs yield $\lambda \nu-\mathrm{PCF}^{+}$. definable embedding-projections when fed them. Furthermore, using 'the $x . e$ ' expressions, we get a $\lambda \nu-\mathrm{PCF}^{+}$-definable embedding-projection pair from name to name $\rightarrow$ nat, via

$$
\begin{aligned}
& e \triangleq \lambda x: \text { name } \rightarrow \lambda y: \text { name } \rightarrow \text { if } x=y \text { then } 0 \text { else } \mathrm{S} 0 \\
& p \triangleq \lambda(f: \text { name } \rightarrow \text { nat }) \rightarrow \text { the } x .(\text { if zero }(f x) \text { then } \mathrm{T} \\
& \quad \text { else if zero }(\operatorname{pred}(f x)) \text { then } \mathrm{F} \text { else } \mathrm{fix}(\lambda x: \text { bool } \rightarrow x)) .
\end{aligned}
$$

It is also not hard to see that there is a $\lambda \nu-\mathrm{PCF}^{+}$-definable embedding-projection pair from bool to nat.

Using these facts, it follows by induction on the structure of $\sigma_{2} \in$ Styp that for all $\sigma_{1} \in \mathrm{Styp}$, there is a $\lambda \nu$ - $\mathrm{PCF}^{+}$-definable embedding-projection pair from $\sigma_{1} \rightarrow \sigma_{2}$ to some $\sigma \in$ Styp. (For example, when $\sigma_{2}=$ name, then we can take $\sigma=\sigma_{1} \times$ name $\rightarrow$ nat.) Using this, one can proceed by induction on the structure of $\tau \in$ Typ to show that there is a $\lambda \nu$ - $\mathrm{PCF}^{+}$-definable embedding-projection pair from $\tau$ to some simple type.

LEMMA 8.9. If there is a $\lambda \nu$ - $\mathrm{PCF}^{+}$-definable embedding-projection pair from $\tau$ to $\sigma$, then uniform-compact definability at $\sigma$ implies the same at $\tau$, i.e. $\left(\mathrm{DEF}_{\sigma}\right) \Rightarrow\left(\mathrm{DEF}_{\tau}\right)$.

Proof. Let $e$ and $p$ be as in Lemma 8.8 and suppose we are given $u \in \mathrm{K} \llbracket \tau \rrbracket$. By Abramsky and Jung [1994, Proposition 3.1.16] we get that $\llbracket e \rrbracket u \in \mathrm{K} \llbracket \sigma \rrbracket$ and by $\left(\mathrm{DEF}_{\sigma}\right)$ we know that there is a $\emptyset \vdash e^{\prime}: \sigma$ such that $\llbracket e^{\prime} \rrbracket=\llbracket e \rrbracket u$. It follows that $p e^{\prime}$ defines $u$, since $\llbracket p e^{\prime} \rrbracket=\llbracket p \rrbracket \llbracket e^{\prime} \rrbracket=\llbracket p \rrbracket(\llbracket e \rrbracket u)=u$.

Combining Lemma 8.8 and Lemma 8.9 with the argument in the survey by Curien [2007, Criterion 2.2], we know that to prove full abstraction for $\lambda \nu-\mathrm{PCF}^{+}$(Theorem 8.5) it suffices to show that $\left(\mathrm{DEF}_{\sigma}\right)$ holds for all simple types $\sigma \in$ Styp.

The proof of $\left(\mathrm{DEF}_{\sigma}\right)$ in principle follows the structure of the traditional proof of uniform-compact definability by Plotkin [1977, Lemma 4.5]. A modern account of this proof can be found in Streicher [2006, Theorem 13.9]. However, in our nominal setting many uses of finite subsets in the traditional proof are replaced by uses of orbit-finite subsets and their presentation as hulls (Theorem 4.5). The definition of hull ${ }_{A} F$ involves existential quantification over finite permutations of $\mathbb{A}$, and for the definability proof we need to reduce this to existential quantification over elements of $\mathbb{A}$. This is where the presence of ex x.e expressions in $\lambda \nu-\mathrm{PCF}^{+}$gets used, in order to prove the following two crucial lemmas. Neither is trivial to prove; the arguments can be found in Appendices $\mathrm{B}$ and $\mathrm{C}$. In particular Lemma 8.11 works by a subtle case distinction over all the different ways the atomic names in the supports of $u$ and $u^{\prime}$ can overlap.

Lemma 8.10. Recall from (8) the definition of step-functions. For each $\tau \in$ Typ, $u \in \mathrm{K} \llbracket \tau \rrbracket$ and $A \subseteq_{\mathrm{f}} \mathbb{A}$, if the step-function ( $u \searrow$ true) $\in \llbracket \tau \rightarrow$ bool $\rrbracket$ is $\lambda \nu$-PCF ${ }^{+}$-definable, then so is $\bigsqcup \operatorname{hull}_{A}\{(u \searrow$ true $)\}$. (Note that the orbit-finite subset hull $A_{A}\{(u \searrow$ true) $\}$ of the nominal Scott domain $\llbracket \tau \rightarrow$ bool $\rrbracket=\llbracket \tau \rrbracket \rightarrow 2_{\perp}$ is bounded above by the constantly-true function and so has a least upper bound.)

Proof. See Appendix B.

LEMmA 8.11. Suppose that $\tau \in \mathrm{Typ}, u, u^{\prime} \in \mathrm{K} \llbracket \tau \rrbracket$ and $A \subseteq_{\mathrm{f}} \mathbb{A}$ satisfy:

- for all uniform-compact elements $v, v^{\prime} \in \mathrm{K} \llbracket \tau \rrbracket$ that do not have an upper bound in $\llbracket \tau \rrbracket$, the least upper bound ( $v \searrow$ true) $\sqcup\left(v^{\prime} \searrow\right.$ false) (exists and) is $\lambda \nu$-PCF ${ }^{+}$-definable; 
- for all finite permutations $\pi: \mathbb{A} \cong \mathbb{A}$ satisfying $\pi \# A$ (see Definition 4.3), it holds that $u$ and $\pi \cdot u^{\prime}$ do not have an upper bound in $\llbracket \tau \rrbracket$.

Then the least upper bound $\bigsqcup$ hull ${ }_{A}\left\{(u \searrow\right.$ true $),\left(u^{\prime} \searrow\right.$ false $\left.)\right\}$ (exists and) is $\lambda \nu-\mathrm{PCF}^{+}$. definable.

Proof. See Appendix C.

Using these two lemmas one can complete the proof of $\left(\mathrm{DEF}_{\sigma}\right)$ for all simple types $\sigma \in$ Styp (and hence complete the proof of Theorem 8.5) by showing by simultaneous induction on the structure of $\sigma$ that:

- $u$ and $(u \searrow$ true) are definable for all uniform-compact elements $u \in \mathrm{K} \| \sigma \rrbracket$;

- $(u \searrow$ true $) \sqcup\left(u^{\prime} \searrow\right.$ false $)$ is definable whenever $u, u^{\prime} \in \mathrm{K} \llbracket \sigma \rrbracket$ are uniform-compact elements that do not have an upper bound in $\llbracket \sigma \rrbracket$.

Most of the work involved in the proof of these two properties lies in the case for functions types, which for simple types are of the form, $\sigma \rightarrow$ nat. By Theorem 4.5 and 5.5 each uniform-compact element $u$ of $\llbracket \sigma \rightarrow$ nat $\rrbracket$ can be expressed as $u=\bigsqcup$ hull ${ }_{A} F$ for some $A \subseteq_{\mathrm{f}} \mathbb{A}, F=\left\{\left(u_{1} \searrow n_{1}\right), \ldots,\left(u_{k} \searrow n_{k}\right)\right\}, u_{1}, \ldots, u_{k} \in \mathrm{K} \llbracket \sigma \rrbracket$ and $n_{1}, \ldots, n_{k} \in \mathbb{N}$. One has to perform another induction on the size of $F$ and make a case distinction based on the existence of $\left(u^{\prime} \searrow n^{\prime}\right),\left(u^{\prime \prime} \searrow n^{\prime \prime}\right) \in F$ such that for all $\pi \# A$ the uniform-compact elements $u^{\prime}$ and $\pi \cdot u^{\prime \prime}$ have no upper bound in $\llbracket \sigma \rrbracket$. Using Lemmas 8.10 and 8.11 the proof goes through following the structure of Streicher [2006, Theorem 13.9], thereby showing full abstraction for $\lambda \nu-\mathrm{PCF}^{+}$.

Remark 8.12 (generative names). It is worth noting that the full abstraction result in this section depends crucially upon the fact that $\lambda \nu-\mathrm{PCF}^{+}$uses Odersky-style local names, rather than generative ones. In Remark 6.3 we noted that an extension of PCF with the kind of generative local names used for example in the $\nu$-calculus [Pitts and Stark 1993] can be translated into $\lambda \nu$-PCF using continuations. Denotationally, generative names can be modelled adequately in Nsd with a continuation monad, but full abstraction fails in this model, because of the results of Stark [1994, Page 66]. So far the only denotational model of this combination of generative names with higher-order functions that is known to be fully abstract makes use of game semantics [Abramsky et al. 2004; Tzevelekos 2008] and is not extensional, that is, quotienting of game strategies by an equivalence relation is needed to make equality in the model coincide with contextual equivalence.

\section{PNA: PROGRAMMING WITH NAME ABSTRACTIONS}

As mentioned in the Introduction, our motivation for studying nominal Scott domains is to explore the denotational semantics of meta-languages that represent objectlanguage binders using the nominal sets notion of name abstraction [Pitts 2013, Chapter 4]. We have seen in Section 7 that there is a straightforward denotational semantics of locally scoped names combined with arithmetic and higher-order recursive functions using nominal Scott domains and the notion of name restriction operation (Definition 7.1). Now we wish to extend this to cover name abstraction.

To do so, we extend $\lambda \nu$-PCF with constructs for name abstraction $\alpha a . e$ and concretion $e_{1} @ e_{2}$, so that it can serve as meta-language. The resulting programming language is called PNA (Programming with Name Abstractions). To exercise the use of name abstraction for expressing object-level binding PNA also features a representative 'nominal algebraic datatype', namely a type for $\alpha$-equivalence classes of $\lambda$-terms, called term. This datatype comes with three constructors (V for variables, A for applications and L 


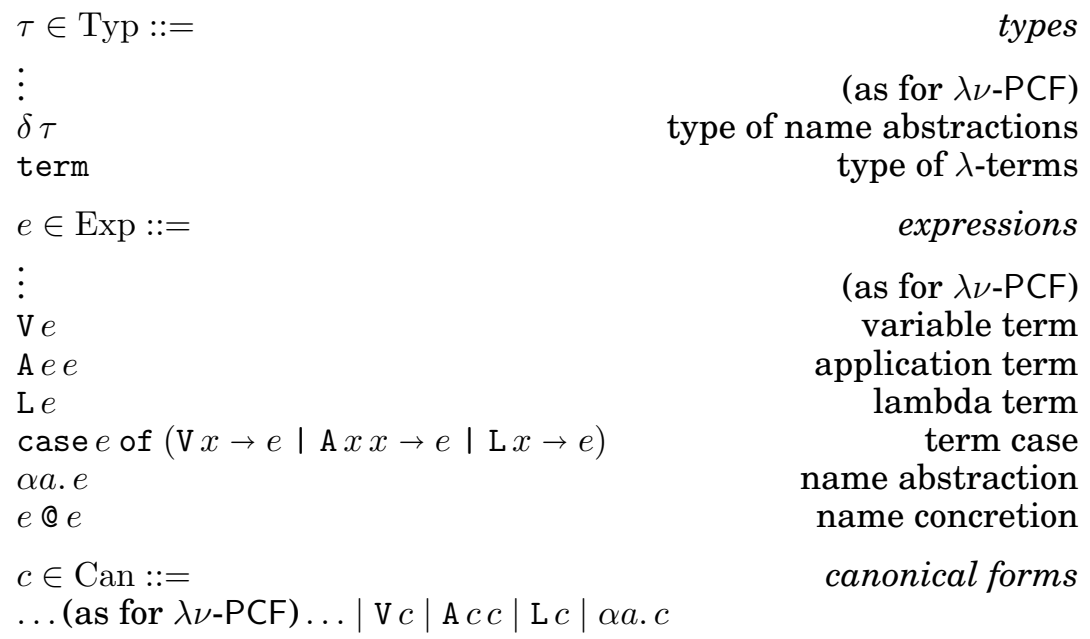

Fig. 7. Syntax of PNA

for $\lambda$-abstractions) and a pattern matching construct

$$
\text { case } e \text { of }\left(\mathrm{V} x_{1} \rightarrow e_{1}\left|\mathrm{~A} x_{2} x_{2}^{\prime} \rightarrow e_{2}\right| \mathrm{L} x_{3} \rightarrow e_{3}\right) \text {. }
$$

Using these new constructs, computation over $\alpha$-equivalence classes of $\lambda$-terms can be expressed directly in PNA. For example, when subst is the PNA expression

$$
\begin{aligned}
& \text { subst } \triangleq \lambda y: \text { term } \rightarrow \lambda x: \text { name } \rightarrow \\
& \operatorname{fix}\left(\lambda(f: \text { term } \rightarrow \text { term }) \rightarrow \lambda y^{\prime}: \text { term } \rightarrow\right. \\
& \text { case } y^{\prime} \text { of } \\
& \quad \mathrm{V} x_{1} \rightarrow \text { if } x_{1}=x \text { then } y \text { else } y^{\prime} \\
& \text { | } \mathrm{A} y_{2} y_{2}^{\prime} \rightarrow \mathrm{A}\left(f y_{2}\right)\left(f y_{2}^{\prime}\right) \\
& \text { | } \mathrm{L} z \rightarrow \mathrm{L}(\alpha a . f(z @ a)))
\end{aligned}
$$

then subst $e_{1} a e_{2}$ computes the $\lambda$-term obtained by capture-avoiding substitution of the $\lambda$-term represented by $e_{1}$ for all free occurrences of the variable named $a$ in the $\lambda$-term represented by $e_{2}$.

The syntax and typing rules for PNA, extending those for $\lambda \nu$-PCF, are given in Figures 7 and 8 . The language's binding forms are

$$
\lambda x: \tau \rightarrow_{-} \quad \nu a ._{-} \quad \text { case } e \text { of }\left(\mathrm{V} x \rightarrow_{-}\left|\mathrm{A} x x \rightarrow_{-}\right| \mathrm{L} \mathrm{L} x \rightarrow_{-}\right) \quad \alpha a ._{-}
$$

and as before, we identify PNA expressions up to $\alpha$-equivalence of bound identifiers, be they variables $(x)$, or atomic names $(a)$.

The operational semantics of PNA is given in Figures 9 and 10. As for $\lambda \nu$-PCF, we choose $\nu a . a$ to be a stuck expression that does not evaluate to any canonical form and whose denotation is $\perp$. We also choose to evaluate under name abstractions, so that $\alpha a . e$ is in canonical form if and only if $e$ is. ${ }^{5}$ These two choices permit a representation of $\alpha$-equivalence classes of $\lambda$-terms in PNA that is as simple as PCF's representation of numbers: they are in bijection with variable-closed canonical forms of type term.

\footnotetext{
${ }^{5}$ It is certainly possible to give a different operational semantics in which one does not evaluate under $\alpha$ abstractions. The corresponding denotational semantics would make more use of lifting than does the one in Section 11.
} 


$$
\begin{aligned}
& \ldots \text { (as for } \lambda \nu \text {-PCF }) \ldots \\
& \frac{\Gamma \vdash e: \text { name }}{\Gamma \vdash \mathrm{V} e: \text { term }} \quad \frac{\Gamma \vdash e_{1}: \text { term } \quad \Gamma \vdash e_{2}: \text { term }}{\Gamma \vdash \mathrm{A} e_{1} e_{2}: \text { term }} \quad \frac{\Gamma \vdash e: \delta \text { term }}{\Gamma \vdash \mathrm{L} e: \text { term }} \\
& \Gamma \vdash e: \text { term } \\
& \frac{\Gamma, x_{1}: \text { name } \vdash e_{1}: \tau \quad \Gamma, x_{2}: \text { term, } x_{2}^{\prime}: \text { term } \vdash e_{2}: \tau \quad \Gamma, x_{3}: \delta \text { term } \vdash e_{3}: \tau}{\Gamma \vdash \text { case } e \text { of }\left(\mathrm{V} x_{1} \rightarrow e_{1}\left|\mathrm{~A} x_{2} x_{2}^{\prime} \rightarrow e_{2}\right| \mathrm{L} x_{3} \rightarrow e_{3}\right): \tau} \\
& \frac{a \in \mathbb{A} \quad \Gamma \vdash e: \tau}{\Gamma \vdash \alpha a . e: \delta \tau} \quad \frac{\Gamma \vdash e_{1}: \delta \tau \quad \Gamma \vdash e_{2}: \text { name }}{\Gamma \vdash e_{1} @ e_{2}: \tau}
\end{aligned}
$$

Fig. 8. PNA typing rules

$\ldots($ as for $\lambda \nu$-PCF $) \ldots$

$$
\begin{aligned}
& \frac{e \Downarrow c}{\mathrm{~V} e \Downarrow \mathrm{V} c} \quad \frac{e_{1} \Downarrow c_{1} \quad e_{2} \Downarrow c_{2}}{\mathrm{~A} e_{1} e_{2} \Downarrow \mathrm{A} c_{1} c_{2}} \quad \frac{e \Downarrow c}{\mathrm{~L} e \Downarrow \mathrm{L} c} \quad \frac{e \Downarrow \mathrm{V} c \quad e_{1}\left[c / x_{1}\right] \Downarrow c^{\prime}}{\operatorname{case} e \text { of }\left(\mathrm{V} x_{1} \rightarrow e_{1} \mid \cdots\right) \Downarrow c^{\prime}} \\
& \frac{e \Downarrow \mathrm{A} c c^{\prime} \quad e_{2}\left[c / x_{2}, c^{\prime} / x_{2}^{\prime}\right] \Downarrow c^{\prime \prime}}{\operatorname{case} e \text { of }\left(\cdots\left|\mathrm{A} x_{2} x_{2}^{\prime} \rightarrow e_{2}\right| \cdots\right) \Downarrow c^{\prime \prime}} \quad \frac{e \Downarrow \mathrm{L} c \quad e_{3}\left[c / x_{3}\right] \Downarrow c^{\prime}}{\operatorname{case} e \text { of }\left(\cdots \mid \mathrm{L} x_{3} \rightarrow e_{3}\right) \Downarrow c^{\prime}} \\
& \frac{e \Downarrow c}{\alpha a . e \Downarrow \alpha a . c} \quad \frac{e_{1} \Downarrow \alpha a . c \quad e_{2} \Downarrow a^{\prime} \quad a \neq a^{\prime} \quad \nu a .\left(a \rightleftharpoons a^{\prime}\right) c \Downarrow c^{\prime}}{e_{1} @ e_{2} \Downarrow c^{\prime}}
\end{aligned}
$$

Fig. 9. PNA evaluation rules

$\ldots$ (as for $\lambda \nu$-PCF) ...

$$
\frac{a \| c:=c^{\prime}}{a \| \mathrm{V} c:=\mathrm{V} c^{\prime}} \quad \frac{a\left\|c_{1}:=c_{1}^{\prime} \quad a\right\| c_{2}:=c_{2}^{\prime}}{a \backslash \| \mathrm{A} c_{1} c_{2}:=\mathrm{A} c_{1}^{\prime} c_{2}^{\prime}} \quad \frac{a \| c:=c^{\prime}}{a \| \mathrm{L} c:=\mathrm{L} c^{\prime}} \quad \frac{a \| c:=c^{\prime} \quad a \neq a^{\prime}}{a \| \alpha a^{\prime} \cdot c:=\alpha a^{\prime} \cdot c^{\prime}}
$$

Fig. 10. PNA partial operation of name restriction

Computing with name abstractions involves deconstructing them, and to do so PNA features a concretion operation $e @ e^{\prime}$ whose operational behaviour is given by the last rule in Figure 9. This rule might be surprising at first, but it will make sense once we consider the denotational semantics of name abstraction and concretion in the next section, in particular the second case of (52).

\section{ABSTRACTION AND CONCRETION}

The results in this section are the basis for giving denotational semantics using nominal Scott domains to languages with name abstraction operations, such as PNA from Section 9 .

THEOREM 10.1. If $D$ is a nominal Scott domain, then so is the nominal poset $[\mathbb{A}] D$ from Definition 3.1. The operation of name abstraction $(a, d) \mapsto\langle a\rangle$ d extends to a morphism $\mathbb{A}_{\perp} \times D \rightarrow[\mathbb{A}] D$ in Nsd once we define $\langle\perp\rangle d \triangleq \perp$. 
Proof. If $S \in \mathrm{P}_{\mathrm{fs}}([\mathbb{A}] D)$ is uniform-directed, then so is $\{d \in D \mid\langle a\rangle d \in S\} \in \mathrm{P}_{\mathrm{fs}} D$, for any $a \in \mathbb{A}$. The same holds if $S$ is finitely supported and bounded from above. In all cases, picking any $a \# S$, one finds that the least upper bound of $S$ in $[\mathbb{A}] D$ is $\langle a\rangle(\bigsqcup\{d \in D \mid\langle a\rangle d \in S\})$. Thus, $[\mathbb{A}] D$ has uniform-directed least upper bounds and least upper bounds of bounded finitely supported subsets, and its least element is $\langle a\rangle \perp$ (for any $a \in \mathbb{A}$ ). The above description of uniform-directed least upper bounds in $[\mathbb{A}] D$ implies that $\langle a\rangle u \in \mathrm{K}(\langle\mathbb{A}\rangle D)$ if and only if $u \in \mathrm{K} D$ and (hence) that $[\mathbb{A}] D$ is $\omega$-algebraic. It also implies that each $\lambda d \in D \rightarrow\langle a\rangle d$ is uniform-continuous.

Given a nominal Scott domain $D$ and $d \in[\mathbb{A}] D$, for each $a \in \mathbb{A}$ with $a \# d$ there is a unique element $d @ a \in D$ satisfying $d=\langle a\rangle(d @ a)$, called the concretion [Pitts 2013 , Section 4.3] of the name abstraction $d$ at the atomic name $a$. Note that this operation is partially defined: to form $d @ a$ we require that $a$ not be in the support of $d$. For flat domains we can make concretion a total, uniform-continuous function simply by mapping the pairs where concretion is undefined to $\perp \in D$. However, for non-flat domains this is not possible, because in general it does not give a monotone function. For example in $[\mathbb{A}]\left(\mathrm{P}_{\mathrm{fs}_{\mathrm{s}}} \mathbb{A}\right), a^{\prime} \in \operatorname{supp}\left(\langle a\rangle\left\{a, a^{\prime}\right\}\right)$ (assuming $\left.a \neq a^{\prime}\right)$, but we cannot define the concretion of $\langle a\rangle\left\{a, a^{\prime}\right\}$ at $a^{\prime}$ to be the least element $\emptyset$ of $\mathrm{P}_{\mathrm{fs}} \mathbb{A}$ since $\langle a\rangle\{a\} \sqsubseteq\langle a\rangle\left\{a, a^{\prime}\right\}$ and $(\langle a\rangle\{a\}) @ a^{\prime}=\left\{a^{\prime}\right\} \neq \emptyset$.

One way to deal with this partiality of concretion in a programming language is to enhance its type system with 'freshness assumptions' to ensure statically that name abstractions are only concreted at fresh atomic names. This is the solution adopted by the original version of FreshML [Pitts and Gabbay 2000] and is the one chosen by Winskel and Turner in their language Nominal HOPLA [Turner and Winskel 2009; Turner 2009]. Later versions of FreshML use a conventional type system and enforce freshness conditions dynamically via the use of generative local names in expressions [Shinwell et al. 2003] — at the expense of purity [Pottier 2007]. We do the same with the language PNA, but achieve purity via the use of Odersky-style local names [Odersky 1994] rather than generative ones. The meaning of this form of local name construct is type-directed and we used Theorem 7.2 to give its denotational semantics for $\lambda \nu$-PCF's types. This extends to PNA types because of the following result.

THEOREM 10.2. If $D \in \mathrm{Nsd}$ possesses a uniform-continuous name restriction operation (Definition 7.1), then the name abstraction domain $[\mathbb{A}] D$ also has one and it satisfies

$$
a \backslash\left(\left\langle a^{\prime}\right\rangle d\right)=\left\langle a^{\prime}\right\rangle(a \backslash d) \quad \text { if } a \neq a^{\prime}
$$

for all $a, a^{\prime} \in \mathbb{A}$ and $d \in D$.

PROOF. First note that (51) uniquely defines name restriction for name abstraction domains because given $a \in \mathbb{A}$, we can always choose a representative for the equivalence class $\left\langle a^{\prime}\right\rangle d$ with $a \neq a^{\prime}$. The rest of the proof, that is showing that (29)-(32) hold, is a straightforward generalisation to nominal Scott domains of the corresponding result for the name abstraction operation on nominal sets [Pitts 2013, Theorem 9.18].

Following Pitts [2011, Corollary 2.14] and assuming $D \in$ Nsd has a uniformcontinuous name restriction operation, we can extend the partial operation of concretion to a total equivariant function _ $@^{t}:[\mathbb{A}] D \times \mathbb{A}_{\perp} \rightarrow D$ satisfying

$$
\left.\begin{array}{l}
(\langle a\rangle d) @^{t} a=d \\
(\langle a\rangle d) @^{t} a^{\prime}=a \backslash\left(a a^{\prime}\right) \cdot d \quad \text { if } a \neq a^{\prime} \\
(\langle a\rangle d) @^{t} \perp=\perp .
\end{array}\right\}
$$

The fact that this is uniform-continuous and hence determines a morphism in Nsd follows from the description of uniform-directed least upper bounds in $[\mathbb{A}] D$ in the 


$$
\begin{aligned}
& \ldots \text { (as for } \lambda \nu \text {-PCF) } \ldots \\
& \llbracket \mathrm{V} e \rrbracket \rho=\left\{\begin{array}{ll}
{[a]_{\alpha}} & \text { if } \llbracket e \rrbracket \rho=a \\
\perp & \text { otherwise }
\end{array} \quad \llbracket \mathrm{A} e_{1} e_{2} \rrbracket \rho= \begin{cases}{\left[t_{1} t_{2}\right]_{\alpha}} & \text { if } \llbracket e_{i} \rrbracket \rho=\left[t_{i}\right]_{\alpha} \\
\perp & \text { otherwise }\end{cases} \right. \\
& \llbracket \mathrm{L} e \rrbracket \rho= \begin{cases}{[\lambda a . t]_{\alpha}} & \text { if } \llbracket e \rrbracket \rho=\langle a\rangle[t]_{\alpha} \\
\perp & \text { otherwise }\end{cases}
\end{aligned}
$$

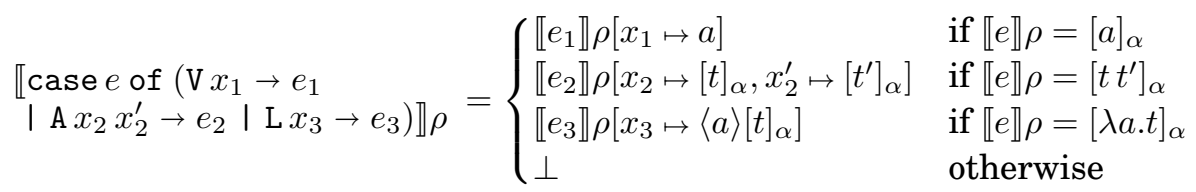

$$
\begin{aligned}
& \llbracket \alpha a . e \rrbracket \rho=\langle a\rangle(\llbracket e \rrbracket \rho) \text { if } a \# \rho \quad \llbracket e_{1} @ e_{2} \rrbracket \rho=\left(\llbracket e_{1} \rrbracket \rho\right) @{ }^{t}\left(\llbracket e_{2} \rrbracket \rho\right)
\end{aligned}
$$

Fig. 11. PNA denotational semantics

proof of Theorem 10.1. For fresh names, the total concretion operation agrees with the 'usual' concretion

$$
(\forall d \in[\mathbb{A}] D) a \# d \Rightarrow d @^{t} a=d @ a .
$$

We will use the total concretion morphism to interpret name concretion expressions in the denotational semantics of PNA.

\section{DENOTATIONAL SEMANTICS OF PNA}

As for $\lambda \nu$-PCF, types in PNA denote nominal Scott domains and well-typed expressions $\Gamma \vdash e: \tau$ denote uniform-continuous functions $\llbracket e \rrbracket \in \llbracket \Gamma \rrbracket \rightarrow \llbracket \tau \rrbracket$. Denotations for typing environments $\Gamma$, booleans bool, numbers nat, product types $\tau_{1} \times \tau_{2}$ and function types $\tau_{1} \rightarrow \tau_{2}$ are the same as the $\lambda \nu$-PCF definitions in Section 7 . The type of $\lambda$-terms term and the type of name abstractions $\delta \tau$ are denoted as follows:

- term $\rrbracket=\left(\Lambda_{\alpha}\right)_{\perp}$, the flat domain on the nominal set of $\alpha$-equivalence classes of $\lambda$-terms [Pitts 2013, Section 4.1],

$$
\Lambda_{\alpha} \triangleq\{t::=a|\lambda a . t| t t\} /=_{\alpha} \quad(\text { where } a \in \mathbb{A}) .
$$

$-\llbracket \delta \tau \rrbracket=[\mathbb{A}] \llbracket \tau \rrbracket$, the domain of name abstractions of the nominal Scott domain $\llbracket \tau \rrbracket$ (Theorem 10.1).

Figure 11 gives the additional definitions for the denotations of PNA expressions. In the clauses involving expressions of type term, we use $[t]_{\alpha}$ to denote the $\alpha$-equivalence class of the syntax tree $t$ of a $\lambda$-term. By Theorems 7.2 and 10.2 the denotation of every PNA-type has a uniform-continuous name restriction operation. This operation is used in the definitions of $\llbracket \nu a . e \rrbracket$ and $e_{1} @ e_{2}$, where for the latter we use the total concretion morphism from (52). Note that the side condition in the clause for $\alpha a$. e can always be satisfied, because we identify expression up to $\alpha$-equivalence. The $\lambda \nu$-PCF results from Section 7 carry over to PNA.

LEMMA 11.1 (PNA SOUNDNESS). If $e \Downarrow c$, then $\llbracket e \rrbracket=\llbracket c \rrbracket$.

PRoOF. The Substitution Lemma, Equivariance Lemma and Restriction Lemma mentioned in the proof of Lemma 7.4 extend from $\lambda \nu$-PCF to PNA. Soundness follows then by rule induction on $e \Downarrow c$. 
THEOREM 11.2 (PNA COMPUTATIONAL ADEQUACY). The PNA contextual preorder $\left(\Gamma \vdash e \leq_{\mathrm{PNA}} e^{\prime}: \tau\right)$ and contextual equivalence $\left(\Gamma \vdash e \cong_{\mathrm{PNA}} e^{\prime}: \tau\right)$ are defined as for $\lambda \nu$-PCF (Definition 6.4). For any $\Gamma \vdash e: \tau$ and $\Gamma \vdash e^{\prime}: \tau$, if $\llbracket e \rrbracket \sqsubseteq \llbracket e^{\prime} \rrbracket \in \llbracket \Gamma \rrbracket \rightarrow \llbracket \tau \rrbracket$, then $\Gamma \vdash e \leq_{\text {PNA }} e^{\prime}: \tau$, and hence we have also that $\llbracket e \rrbracket=\llbracket e^{\prime} \rrbracket \in \llbracket \Gamma \rrbracket \rightarrow \llbracket \tau \rrbracket$ implies $\Gamma \vdash e \cong$ PNA $e^{\prime}: \tau$.

Proof. The overall structure of the proof is the same as in Theorem 7.5, but the presence of name abstraction and concretion creates complications: see Appendix D.

\section{FULL ABSTRACTION FOR PNA ${ }^{+}$}

In this section we show that the full abstraction result from Section 8 can be extended to include computation with name abstractions. With some tweaks and extensions, the proof goes through as for $\lambda \nu-\mathrm{PCF}^{+}$. We extended $\lambda \nu$-PCF with existential name quantification and definite name description in order to gain enough expressivity for full abstraction. As Theorem 12.2 shows, the same extensions suffice for PNA.

Definition 12.1. $\mathrm{PNA}^{+}$is the extension of PNA with constructs for existential name quantification and definite name description, as they are given in Figure 6: $\mathrm{PNA}^{+}=$ PNA + ex + the. Similarly we define the languages PNA+ex and PNA+the. The contextual preorder relations $\leq_{\mathrm{PNA}^{+}}, \leq_{\mathrm{PNA}+\mathrm{ex}}$ and $\leq_{\mathrm{PNA}+\text { the }}$ are given as in Definition 6.4.

The results of Appendix A can be extended to PNA, giving us the equivalents of (49) and (50)

$$
\begin{aligned}
& \emptyset \vdash \mathrm{F}_{1} \leq_{\mathrm{PNA}+\text { the }} \mathrm{F}_{2}:((\text { name } \rightarrow \text { bool }) \rightarrow \text { bool }) \rightarrow \text { bool } \\
& \emptyset \vdash \mathrm{G}_{1} \leq_{\mathrm{PNA}+\text { ex }} \mathrm{G}_{2}:((\text { name } \rightarrow \text { bool }) \rightarrow \text { name }) \rightarrow \text { bool } .
\end{aligned}
$$

Arguing as in the proof of Theorem 8.4, this shows that PNA+ex and PNA+the fail to be fully abstract. More positively, Theorem 8.5 can be adjusted to the current setting, showing that $\mathrm{PNA}^{+}$is fully abstract.

THEOREM 12.2 (FULL ABSTRACTION FOR PNA ${ }^{+}$). For all well-typed expressions $\Gamma \vdash e: \tau$ and $\Gamma \vdash e^{\prime}: \tau$ in $\mathrm{PNA}^{+}$, we have

$$
\llbracket e \rrbracket \sqsubseteq \llbracket e^{\prime} \rrbracket \in \llbracket \Gamma \rrbracket \rightarrow \llbracket \tau \rrbracket \Leftrightarrow \Gamma \vdash e \leq_{\mathrm{PNA}^{+}} e^{\prime}: \tau
$$

and hence also $\llbracket e \rrbracket=\llbracket e^{\prime} \rrbracket \in \llbracket \Gamma \rrbracket \rightarrow \llbracket \tau \rrbracket \Leftrightarrow \Gamma \vdash e \cong_{\mathrm{PNA}^{+}} e^{\prime}: \tau$.

In the rest of this section we sketch how the proof of Theorem 8.5 can be extended to a proof of Theorem 12.2.

As for $\lambda \nu-\mathrm{PCF}^{+}$, we reduce the full abstraction property $\left(\mathrm{PNA}^{+}-\mathrm{FA}_{\tau}\right)$ at arbitrary $\mathrm{PNA}^{+}$types to this property for simple types, using the same definition of 'simple type' as before (Definition 8.7). However, the analogue of Lemma 8.8 for PNA ${ }^{+}$fails: it is not the case that there is a definable embedding-projection pair from each $\mathrm{PNA}^{+}$type to some simple type (see the example given below in Open Problem 12.5). However, the property holds for definable retracts instead of definable embedding-projection pairs and this suffices for our proof.

LEMMA 12.3. A type $\tau$ is a $\mathrm{PNA}^{+}$-definable retract of a type $\sigma$ if there are closed $\mathrm{PNA}^{+}$-expressions $\emptyset \vdash i: \tau \rightarrow \sigma$ and $\emptyset \vdash r: \sigma \rightarrow \tau$ so that $\llbracket \lambda x: \tau \rightarrow r(i x) \rrbracket=i d_{\llbracket \tau \rrbracket}$. It holds that every $\mathrm{PNA}^{+}$type $\tau \in$ Typ is a is a $\mathrm{PNA}^{+}$-definable retract of some $\sigma \in$ Styp.

PROOF. Since name abstraction satisfies a form of $\eta$-expansion $(\llbracket \alpha a .(e @ a) \rrbracket=\llbracket e \rrbracket$, if $a \notin \mathrm{fn}(e))$, each type $\delta \tau$ is a $\mathrm{PNA}^{+}$-definable retract of name $\rightarrow \tau$ via

$$
\begin{aligned}
& i \triangleq \lambda(x: \delta \tau) \rightarrow \lambda y: \text { name } \rightarrow x @ y \\
& r \triangleq \lambda(f: \text { name } \rightarrow \tau) \rightarrow \alpha a . f a
\end{aligned}
$$


(cf. Pitts [2011, Theorem 2.13]). It follows that if $\tau$ is a PNA ${ }^{+}$-definable retract of a simple type, then so is $\delta \tau$ (using the fact implicit in the proof of Lemma 8.8 that if $\sigma_{1}$ and $\sigma_{2}$ are simple, then $\sigma_{1} \rightarrow \sigma_{2}$ is a definable retract of a simple type).

Using a suitable Gödel-numbering of $\lambda$-terms in the presence of an environment consisting of a finite list of distinct atomic names (giving the free variables of the $\lambda$ term) one can exhibit term as a $\mathrm{PNA}^{+}$-definable retract of the simple type $\sigma \times$ nat, where the second component nat is used to codes the term's syntax tree and the first component $\sigma \triangleq$ nat $\times$ (name $\rightarrow$ nat) is used to code environments as a pair consisting of list-length and a function (taking value 0 at all but finitely many arguments) giving positions in the list.

Every embedding-projection pair forms a retract, so we can combine these observations about $\delta \tau$ and term with the argument in the proof of Lemma 8.8 to conclude that every PNA ${ }^{+}$-type is a definable retract of a simple type.

We can use the above lemma to show that definability at simple types implies full abstraction at all types.

Lemma 12.4. It holds that ( $\forall \sigma \in \mathrm{Styp})\left(\mathrm{DEF}_{\sigma}\right)$ implies $(\forall \tau \in \mathrm{Typ})\left(\mathrm{PNA}^{+}-\mathrm{FA}_{\tau}\right)$.

Proof. The left-to-right direction of $\left(\mathrm{PNA}^{+}-\mathrm{FA}_{\tau}\right)$ of course holds already by computational adequacy (Theorem 11.2). We prove the right-to-left direction first for closed expressions of simple type, that is $\llbracket e \rrbracket \nsubseteq \llbracket e^{\prime} \rrbracket \in \llbracket \sigma \rrbracket \Rightarrow \emptyset \vdash e \mathbb{\text { PNA }}^{+} e^{\prime}: \sigma$. This proof is straight-forward by following the classical definability argument as surveyed by Curien [2007, Criterion 2.2].

For showing the property for open expressions of simple type $\llbracket e \rrbracket \nsubseteq \llbracket e^{\prime} \rrbracket \in \llbracket \Gamma \rrbracket \rightarrow \llbracket \sigma \rrbracket \Rightarrow$ $\Gamma \vdash e \mathbb{Z N N A}_{\mathrm{PN}^{+}} e^{\prime}: \sigma$, we need to take into account that the environment $\Gamma=\left\{x_{1}:\right.$ $\left.\tau_{1}, \ldots, x_{n}: \tau_{n}\right\}$ may contain non-simple types. By Lemma 12.3 we know that there is a $\sigma \in$ Styp such that $\tau_{1} \times \ldots \times \tau_{n}$ is a definable retract of $\sigma$, say with expressions $i$ and $r$. It follows from $\llbracket e \rrbracket \nsubseteq \llbracket e^{\prime} \rrbracket$ that there must be a $u \in \mathrm{K} \llbracket \sigma \rrbracket$ (which is definable) such that $\llbracket e \rrbracket\left(\llbracket r \rrbracket u_{1}\right) \nsubseteq \llbracket e \rrbracket\left(\llbracket r \rrbracket u_{1}\right) \in \llbracket \sigma \rrbracket$ and with that we can use the above argument for closed expressions to show $\Gamma \vdash e \mathbb{P}_{\mathrm{PNA}^{+}} e^{\prime}: \sigma$.

What is left to prove is that if $\tau$ is a definable retract of $\sigma$, then $\left(\mathrm{PNA}^{+}-\mathrm{FA}_{\sigma}\right)$ implies $\left(\mathrm{PNA}^{+}-\mathrm{FA}_{\tau}\right)$. Suppose $\left(\mathrm{PNA}^{+}-\mathrm{FA}_{\sigma}\right)$ holds and that $\Gamma \vdash e \leq_{\mathrm{PNA}^{+}} e^{\prime}: \tau$. Then we have $\Gamma \vdash i e \leq_{\mathrm{PNA}^{+}} i e^{\prime}: \sigma$ by compositionality of $\leq_{\mathrm{PNA}^{+}}$. Thus by $\left(\mathrm{FA}_{\sigma}\right)$, for any $\rho \in \llbracket \Gamma \rrbracket$ we have $\llbracket i \rrbracket(\llbracket e \rrbracket \rho)=\llbracket i e \rrbracket \rho \sqsubseteq \llbracket i e^{\prime} \rrbracket \rho=\llbracket i \rrbracket\left(\llbracket e^{\prime} \rrbracket \rho\right)$. We know that $\llbracket i \rrbracket$ has a monotone left inverse $\llbracket r \rrbracket$, so $\llbracket e \rrbracket \rho \sqsubseteq \llbracket e^{\prime} \rrbracket \rho$. As $\rho$ was chosen arbitrarily we get $\llbracket e \rrbracket \sqsubseteq \llbracket e^{\prime} \rrbracket \in \llbracket \Gamma \rrbracket \rightarrow \llbracket \tau \rrbracket$.

$\lambda \nu-\mathrm{PCF}^{+}$is included in $\mathrm{PNA}^{+}$, so the proof of uniform-compact definability at simple types $(\forall \sigma \in \mathrm{Styp})\left(\mathrm{DEF}_{\sigma}\right)$ for $\lambda \nu-\mathrm{PCF}^{+}$in Section 8 directly translates to $\mathrm{PNA}^{+}$. This completes the proof of full abstraction of $\mathrm{PNA}^{+}$by Lemma 12.4.

We conclude this section with the discussion of some open problems.

Open Problem 12.5. For an arbitrary $\mathrm{PNA}^{+}$type $\tau$, are the uniform-compact elements of $\llbracket \tau \rrbracket \mathrm{PNA}^{+}$-definable?

The definable retract (54) is not in general an embedding-projection pair. For example, when $\tau=$ bool one can calculate that $\llbracket r \rrbracket \in\left(\mathbb{A}_{\perp} \rightarrow 2_{\perp}\right) \rightarrow[\mathbb{A}] 2_{\perp}$ maps the element $e q_{a} \in \mathbb{A}_{\perp} \rightarrow 2_{\perp}$ from Example 5.6 to $\langle a\rangle$ false; and that $\llbracket i \rrbracket$ maps this name abstraction to $k_{\text {false }} \triangleq \lambda d \in \mathbb{A}_{\perp} \rightarrow$ if $d=\perp$ then $\perp$ else false. Since $e q_{a} \nsubseteq k_{\text {false }}$, we do not have that $\llbracket \lambda(x:$ name $\rightarrow$ bool $) \rightarrow i(r x) \rrbracket \sqsubseteq i d_{\llbracket \text { name } \rightarrow \text { bool } \rrbracket}$. So it seems that PNA ${ }^{+}$-types are not definably embeddable into simple types. As a result, we cannot deduce the definability property of Definition 8.6 at arbitrary types from the special case for simple types.

Open Problem 12.6. Is there a fully abstract model of PNA based on games in nominal sets? 
Just as PCF is of more interest from a programming point of view than PCF+por, we regard PNA (suitably extended with recursive types) as a 'pure' version of FreshML that is potentially useful for functional programming with syntactical data involving binders. Game semantics provided an interesting solution for the original full abstraction problem for PCF [Abramsky et al. 2000; Hyland and Ong 2000], and its nominal version has provided computationally useful, fully abstract models of generative local state [Abramsky et al. 2004; Tzevelekos 2008; Laird 2008; Murawski and Tzevelekos 2012]. Can nominal game semantics provide a similar thing for PNA?

Open Problem 12.7. Is there a nominal Scott domain semantics for the form of nominal computation embodied by the $\mathrm{N} \lambda$ language [Bojańczyk et al. 2012]?

With $N \lambda$, Bojańczyk et al. extend the simply-typed $\lambda$-calculus with a collection type representing orbit-finite subsets (Section 2) via a syntax for hulls (Definition 4.3). ${ }^{6}$ It is natural to consider adding fixed point recursion to this language, with a denotational semantics using nominal domains rather than nominal sets. The denotational semantics of such an extension of $\mathrm{N} \lambda$ will require the development of orbit-finite power domains $\mathrm{F}_{\mathrm{n}} D$ in Nsd, whose uniform-compact elements are orbit-finite subsets of the uniform-compact elements of $D$.

Open Problem 12.8. What recursive domain equations can be solved in Nsd?

In his thesis, Shinwell [2005, Section 4.5] shows that the traditional method for constructing minimally invariant solutions for locally continuous functors of mixed variance can be applied to the simple notion of nominal domain given by nominal posets with least upper bounds of finitely supported $\omega$-chains. This can be extended to udcpos: see Pitts [2013, Section 11.4]. An interesting alternative approach is to develop a nominal version of Scott's information systems [Scott 1982] and construct solutions for recursive domain equations via inductively defined nominal sets of information tokens. We have begun to develop such a theory of nominal Scott information systems in which the role of finite sets is replaced by orbit-finite nominal sets. From a logical point of view [Abramsky 1991], nominal information systems are presentations of non-trivial nominal posets with all orbit-finite meets, rather than just finite meets. We expect this machinery can be used to good effect for the orbit-finite power domain construct mentioned above, as well as for a version for nominal Scott domains of Moggi's monad for dynamic allocation [Moggi 1989, Section 4.14] featuring a freely generated uniform-continuous name restriction operation (cf. Pitts [2013, Section 9.5]).

\section{CONCLUSION}

The results in this paper provide further evidence for how a semantic theory (domain theory in this case) is enhanced by using nominal sets: we gain the ability to model constructs involving names and their symmetries while preserving most aspects of the classical theory. The complications arising from the use of nominal sets are feasible and somehow orthogonal to the other developments. At the same time, their use gives access to new constructs that are far from trivial. This is the case for the notion of orbit-finite subset, which formalizes the important idea of finiteness modulo symmetry within nominal sets. We agree with Bojańczyk et al. [2012] that this is an important notion with many potential applications. Here we have used it to develop a nominal domain theory that, via our full abstraction result, has a good fit with higher-type computation involving local names and name abstractions.

${ }^{6}$ Their paper [Bojańczyk et al. 2012] is concerned with general 'Fraïssé nominal sets'. Here we restrict our attention to the 'equality symmetry' and nominal sets in the original sense. 


\section{APPENDIX}

\section{A. PROOFS OF (49) AND (50)}

We first establish some extensionality results for the $\lambda \nu-\mathrm{PCF}^{+}$contextual preorder (Proposition A.3). These results specialise to $\lambda \nu$-PCF + the and $\lambda \nu$-PCF + ex and hence can be used as tools for proving (49) and (50).

Notation A.1. In order to make a better distinction between the programming languages in use, for this section we tag syntactic sets with the languages they belong to. So $\operatorname{Exp}^{\lambda \nu-\mathrm{PCF}^{+}}$is the set of expressions that belong to $\lambda \nu-\mathrm{PCF}^{+}$; ditto $\operatorname{Exp}^{\lambda \nu-\mathrm{PCF}+\text { the }}$ and $\operatorname{Exp}^{\lambda \nu-\mathrm{PCF}+\mathrm{ex}}$, as well as $\mathrm{Can}^{\lambda \nu-\mathrm{PCF}^{+}}$and $\mathrm{Typ}^{\lambda \nu-\mathrm{PCF}^{+}}$. We also extend the logical relation (42) to open expressions $\Gamma \vdash e: \tau$ by defining

$$
d \triangleleft_{\Gamma \mid \tau} e \triangleq(\forall \rho, s) \rho \triangleleft_{\Gamma} s \Rightarrow d \rho \triangleleft_{\tau} e s
$$

where $d \in \llbracket \Gamma \rrbracket \rightarrow \llbracket \tau \rrbracket, \rho$ is a $\Gamma$-valuation, $s$ is a $\Gamma$-substitution and $\triangleleft_{\Gamma}$ is defined in (43).

Lemma A.2. All expressions $e_{1}, e_{2} \in \operatorname{Exp}^{\lambda \nu-\mathrm{PCF}^{+}}$satisfy

$$
\Gamma \vdash e_{1} \leq_{\lambda \nu-\mathrm{PCF}} e_{2}: \tau \Leftrightarrow \llbracket e_{1} \rrbracket \triangleleft_{\Gamma \mid \tau} e_{2} .
$$

PROOF. As noted in Theorem 8.5, the adequacy results in Section 7, in particular the fundamental property of the logical relation (44), extend from $\lambda \nu$-PCF to $\lambda \nu-\mathrm{PCF}^{+}$. We use these results in this and the forthcoming proofs.

Suppose $\llbracket e_{1} \rrbracket \triangleleft_{\Gamma \mid \tau} e_{2}$ and let a context $C[-]$ be given with $\emptyset \vdash C\left[e_{1}\right]:$ bool, $\emptyset \vdash C\left[e_{2}\right]$ : bool and $C\left[e_{1}\right] \Downarrow \mathrm{T}$. Lemma 7.4 implies $\llbracket C\left[e_{1}\right] \rrbracket=$ true. We can show by induction on the structure of $C[]$ that $\llbracket e_{1} \rrbracket \triangleleft_{\Gamma \mid \tau} e_{2} \Rightarrow \llbracket C\left[e_{1}\right] \rrbracket \triangleleft_{\text {bool }} C\left[e_{2}\right]$. Therefore true $\triangleleft_{\text {bool }} C\left[e_{2}\right]$; and hence $C\left[e_{2}\right] \Downarrow \mathrm{T}$, by definition of $\triangleleft_{\text {bool }}$. Thus we obtain $\Gamma \vdash e_{1} \leq_{\lambda \nu}$ PCF $^{+} e_{2}: \tau$.

To show the converse, one proves by induction on $\tau$ that

$$
\left(d \triangleleft_{\Gamma \mid \tau} e_{1} \wedge \Gamma \vdash e_{1} \leq_{\lambda \nu \text {-PCF}}+e_{2}: \tau\right) \Rightarrow d \triangleleft_{\Gamma \mid \tau} e_{2} .
$$

Then we can apply this to $\llbracket e_{1} \rrbracket \triangleleft_{\Gamma \mid \tau} e_{1}$, which holds by the fundamental property (44).

Proposition A.3 (ExTEnsionality). The contextual preorder for $\lambda \nu$-PCF ${ }^{+}$is extensional at functions and ground types for variable-closed expressions. That is for all $e_{1}, e_{2} \in \operatorname{Exp}^{\lambda \nu-\mathrm{PCF}^{+}}$we have that if $\gamma \in\{$ bool, nat, name $\}$, then $\emptyset \vdash e_{1} \leq_{\lambda \nu-\mathrm{PCF}^{+}} e_{2}: \gamma$ holds if and only if

$$
(\forall c) e_{1} \Downarrow c \Rightarrow e_{2} \Downarrow c .
$$

At function types $\emptyset \vdash e_{1} \leq_{\lambda \nu \text {-PCF }} e_{2}: \tau_{1} \rightarrow \tau_{2}$ holds if and only if

$$
(\forall e) \emptyset \vdash e: \tau_{1} \Rightarrow \emptyset \vdash e_{1} e \leq_{\lambda \nu-\mathrm{PCF}^{+}} e_{2} e: \tau_{2} .
$$

PROOF. The only-if directions are immediate from the definition of $\leq_{\lambda \nu-\mathrm{PCF}^{+}}$. Conversely, Lemma A.2 tells us that it is enough to show $\llbracket e_{1} \rrbracket \triangleleft_{\tau} e_{2}$. In the ground type case $\tau=\gamma$, if $\llbracket e_{1} \rrbracket=\perp$ then $\llbracket e_{1} \rrbracket \triangleleft_{\gamma} e_{2}$ follows directly. Otherwise we must have $\llbracket e_{1} \rrbracket=\llbracket c \rrbracket$ which implies $e_{1} \Downarrow c$ by the fundamental property (44) at $e_{1}$. This implies $e_{2} \Downarrow c$ by assumption, and hence $\llbracket e_{1} \rrbracket \triangleleft_{\gamma} e_{2}$, as required. In the function case $\tau=\tau_{1} \rightarrow \tau_{2}$, let any $d \triangleleft_{\tau_{1}} e$ be given. With the fundamental property we get $\llbracket e_{1} \rrbracket \triangleleft_{\tau_{1} \rightarrow \tau_{2}} e_{1}$, so by definition $\llbracket e_{1} \rrbracket d \triangleleft_{\tau_{2}} e_{1} e$. By assumption we know $\emptyset \vdash e_{1} e \leq_{\lambda \nu \text {-PCF }} e_{2} e: \tau_{2}$ and with (55) this gives us $\llbracket e_{1} \rrbracket d \triangleleft_{\tau_{2}} e_{2} e$. This shows $\llbracket e_{1} \rrbracket \triangleleft_{\tau_{1} \rightarrow \tau_{2}} e_{2}$, by the definition of $\triangleleft_{\tau_{1} \rightarrow \tau_{2}}$; and then we can apply Lemma A.2 to get $\emptyset \vdash e_{1} \leq_{\lambda \nu \text {-PCF}}+e_{2}: \tau_{1} \rightarrow \tau_{2}$. 
Extensionality holds with the same proofs also for the contextual preorders of $\lambda \nu$-PCF+the and $\lambda \nu$-PCF+ex. This allows us to deduce (49) from the following property of the $\lambda \nu-\mathrm{PCF}+$ the evaluation relation

$$
a \# e \wedge(\forall c) e\left[\operatorname{eqBot}_{a} / x\right] \Downarrow c \Rightarrow e[\mathrm{kBot} / x] \Downarrow c
$$

that holds for any expression $e \in \operatorname{Exp}^{\lambda \nu-\mathrm{PCF}+\text { the }}$ of type $x:$ name $\rightarrow$ bool $\vdash e:$ bool; eqBot ${ }_{a}$ and $k B o t$ are defined in (20) and (21). In the same way we can use extensionality to deduce (50) from the following property of the $\lambda \nu$-PCF + ex evaluation relation

$$
a \# e \wedge e\left[\mathrm{eq}_{a} / x\right] \Downarrow a^{\prime} \Rightarrow a \neq a^{\prime}
$$

where $e \in \operatorname{Exp}^{\lambda \nu \text {-PCF+ex }}$ with $x:$ name $\rightarrow$ bool $\vdash e:$ name and eq ${ }_{a}$ is defined in (24).

We prove (56) and (57) by reformulating the evaluation relation $\Downarrow$ in terms of a smallstep transition relation in the style of Felleisen and Hieb [1992], but with evaluation contexts formulated as stacks of evaluation frames. We call this type of operational semantics frame-stack semantics, it also known as abstract machine semantics in the literature. (See Pitts [2002] for a discussion of this technique for proving contextual equivalences.)

The frame-stack evaluation relation for $\lambda \nu-\mathrm{PCF}^{+}$

$$
\langle F, e\rangle \rightarrow\left\langle F^{\prime}, e^{\prime}\right\rangle
$$

is defined in Figure 12. We use the name restriction operation from Figure 4 in the clause for $\nu a . e$. For brevity, the cases for ex $x$. e and the x.e use some syntactic sugar: bot $_{\tau}$ from (19) and a pattern matching construct case bool $_{\text {for }}$ boolean tuples, which can be defined by conditionals for any tuple length.

The evaluation relation is formulated in terms of a transition system between configurations. However, a configurations $\langle F, e\rangle$ is not just a pair built from a frame-stack $F \in$ Stack $^{\lambda \nu-\mathrm{PCF}^{+}}$and an expression $e \in \operatorname{Exp}^{\lambda \nu-\mathrm{PCF}^{+}}:$We identify configurations by a form of $\alpha$-equivalence where a binder in a frame (for $\lambda \nu-\mathrm{PCF}^{+}$only the frame $\nu a . \cdot$ ) can bind free identifiers in later frames or the expression. For example $\langle\operatorname{Id} \circ(\nu a . \cdot) \circ(\cdot=a), a\rangle$ is $\alpha$-equivalent to $\left\langle\operatorname{Id} \circ\left(\nu a^{\prime} \cdot \cdot\right) \circ\left(\cdot=a^{\prime}\right), a^{\prime}\right\rangle$, but it is not $\alpha$-equivalent to $\langle\operatorname{Id}, \nu a . a=a\rangle$. This identification is implicit and results in the following equality for configurations

$$
\langle F, e\rangle=\left\langle F^{\prime}, e^{\prime}\right\rangle \Leftrightarrow|F|=\left|F^{\prime}\right| \wedge F[e]=F^{\prime}\left[e^{\prime}\right]
$$

where $|F|$ is the length of the frame-stack $F$. Let Config ${ }^{\lambda \nu}-\mathrm{PCF}^{+}$be the set of all such configurations for $\lambda \nu-\mathrm{PCF}^{+}$. The notions of permutation action, free names, free variables and capture-avoiding substitution extend to configurations in the obvious way, taking into account this form of $\alpha$-equivalence for configurations.

Definition A.4. For each $n \in \mathbb{N}$, let the relation $\rightarrow^{n}$ be defined by

$$
\begin{aligned}
\langle F, e\rangle \rightarrow^{0}\left\langle F^{\prime}, e^{\prime}\right\rangle & \triangleq\langle F, e\rangle=\left\langle F^{\prime}, e^{\prime}\right\rangle \\
\langle F, e\rangle \rightarrow^{n+1}\left\langle F^{\prime}, e^{\prime}\right\rangle & \triangleq\langle F, e\rangle \rightarrow\left\langle F^{\prime \prime}, e^{\prime \prime}\right\rangle \wedge\left\langle F^{\prime \prime}, e^{\prime \prime}\right\rangle \rightarrow^{n}\left\langle F^{\prime}, e^{\prime}\right\rangle
\end{aligned}
$$

and let $\langle F, e\rangle \rightarrow^{*}\left\langle F^{\prime}, e^{\prime}\right\rangle$ be defined to hold if there is a $n \in \mathbb{N}$ such that $\langle F, e\rangle \rightarrow^{n}\left\langle F^{\prime}, e^{\prime}\right\rangle$. The operational semantics from Section 6 and the frame-stack semantics agree:

Proposition A.5. For all $e \in \operatorname{Exp}^{\lambda \nu-\mathrm{PCF}^{+}}$and $c \in \mathrm{Can}^{\lambda \nu-\mathrm{PCF}^{+}}$we have $e \Downarrow c \Leftrightarrow$ $\langle\mathrm{Id}, e\rangle \rightarrow^{*}\langle\mathrm{Id}, c\rangle$.

Proof. We prove $e \Downarrow c \Rightarrow\left(\forall F \in S t a c k^{\lambda \nu-\mathrm{PCF}^{+}}\right)\langle F, e\rangle \rightarrow^{*}\langle F, c\rangle$ by rule induction on $e \Downarrow c$ for the left-to-right direction. The right-to-left direction is a consequence of the more general statement $(\forall n \in \mathbb{N})\langle F, e\rangle \rightarrow^{n}\langle\mathrm{Id}, c\rangle \Rightarrow F[e] \Downarrow c$ (proved by induction on 


$$
\begin{aligned}
& F \in \text { Stack }^{\mathrm{PNA}^{+}}::=\operatorname{Id} \mid F \circ E \\
& E \in \text { Frame }^{\mathrm{PNA}^{+}}::=\text {if } \cdot \text { then } e \text { else } e|\mathrm{~S} \cdot| \text { pred } \cdot \mid \text { zero } \cdot \mid \text { fst } \cdot \mid \text { snd } \cdot \mid \cdot e \\
& |\nu a \cdot \cdot|(\cdot \rightleftharpoons e) e|(a \rightleftharpoons \cdot) e|(a \rightleftharpoons a) \cdot|\cdot=e| a=\text {. } \\
& \left\langle F \text {, if } e_{1} \text { then } e_{2} \text { else } e_{3}\right\rangle \rightarrow\left\langle F \circ \text { if } \cdot \text { then } e_{2} \text { else } e_{3}, e_{1}\right\rangle \\
& \left\langle F \circ \text { if then } e_{2} \text { else } e_{3}, \mathrm{~T}\right\rangle \rightarrow\left\langle F, e_{2}\right\rangle \quad\left\langle F \circ \text { if } \cdot \text { then } e_{2} \text { else } e_{3}, \mathrm{~F}\right\rangle \rightarrow\left\langle F, e_{3}\right\rangle \\
& \langle F, \mathrm{~S} e\rangle \rightarrow\langle F \circ \mathrm{S} \cdot, e\rangle \quad\langle F \circ \mathrm{S} \cdot, c\rangle \rightarrow\langle F, \mathrm{~S} c\rangle \quad\langle F, \text { pred } e\rangle \rightarrow\langle F \circ \text { pred } \cdot, e\rangle \\
& \langle F \circ \text { pred }, \mathrm{S} c\rangle \rightarrow\langle F, c\rangle \quad\langle F, \text { zero } e\rangle \rightarrow\langle F \circ \text { zero }, e\rangle \quad\langle F \circ \text { zero }, 0\rangle \rightarrow\langle F, \mathrm{~T}\rangle \\
& \langle F \circ \text { zero }, \mathrm{S} c\rangle \rightarrow\langle F, \mathrm{~F}\rangle \quad\langle F, \text { fst } e\rangle \rightarrow\langle F \circ \text { fst } \cdot, e\rangle \quad\left\langle F \circ \text { fst } \cdot,\left(e_{1}, e_{2}\right)\right\rangle \rightarrow\left\langle F, e_{1}\right\rangle \\
& \langle F, \text { snd } e\rangle \rightarrow\langle F \circ \text { snd } \cdot, e\rangle \quad\left\langle F \circ \text { snd } \cdot,\left(e_{1}, e_{2}\right)\right\rangle \rightarrow\left\langle F, e_{2}\right\rangle \quad\left\langle F, e_{1} e_{2}\right\rangle \rightarrow\left\langle F \circ \cdot e_{2}, e_{1}\right\rangle \\
& \left\langle F \circ \cdot e_{2}, \lambda x: \tau \rightarrow e\right\rangle \rightarrow\left\langle F, e\left[e_{2} / x\right]\right\rangle \quad\langle F, \text { fix } e\rangle \rightarrow\langle F, e(\text { fix } e)\rangle \\
& \langle F, \nu a . e\rangle \rightarrow\langle F \circ \nu a . \cdot, e\rangle \quad\langle F \circ \nu a . \cdot, c\rangle \rightarrow\left\langle F, c^{\prime}\right\rangle \quad \text { if } a \| c:=c^{\prime} \\
& \left\langle F,\left(e_{1} \rightleftharpoons e_{2}\right) e_{3}\right\rangle \rightarrow\left\langle F \circ\left(\cdot \rightleftharpoons e_{2}\right) e_{3}, e_{1}\right\rangle \quad\left\langle F \circ\left(\cdot \rightleftharpoons e_{2}\right) e_{3}, a_{1}\right\rangle \rightarrow\left\langle F \circ\left(a_{1} \rightleftharpoons \cdot\right) e_{3}, e_{2}\right\rangle \\
& \left\langle F \circ\left(a_{1} \rightleftharpoons \cdot\right) e_{3}, a_{2}\right\rangle \rightarrow\left\langle F \circ\left(a_{1} \rightleftharpoons a_{2}\right) \cdot, e_{3}\right\rangle \quad\left\langle F \circ\left(a_{1} \rightleftharpoons a_{2}\right) \cdot c\right\rangle \rightarrow\left\langle F,\left(a_{1} a_{2}\right) \cdot c\right\rangle \\
& \left\langle F, e_{1}=e_{2}\right\rangle \rightarrow\left\langle F \circ \cdot=e_{2}, e_{1}\right\rangle \quad\left\langle F \circ \cdot=e_{2}, a_{1}\right\rangle \rightarrow\left\langle F \circ a_{1}=\cdot, e_{2}\right\rangle \\
& \left\langle F \circ a_{1}=\cdot, a_{2}\right\rangle \rightarrow\langle F, \mathrm{~T}\rangle \quad \text { if } a_{1}=a_{2} \quad\left\langle F \circ a_{1}=\cdot, a_{2}\right\rangle \rightarrow\langle F, \mathrm{~F}\rangle \quad \text { if } a_{1} \neq a_{2} \\
& \langle F, \text { ex } x . e\rangle \rightarrow\left\langle F, \text { if } e[a / x] \text { then } \mathrm{T} \text { else bot } \mathrm{bool}_{\mathrm{bol}}\right\rangle \quad \text { for all } a \in \mathbb{A} \\
& \langle F, \text { ex } x . e\rangle \rightarrow\left\langle F, \text { case }_{\text {bool }}\left(e\left[a_{1} / x\right], \ldots, e\left[a_{n} / x\right]\right) \text { of }\left((\mathrm{F}, \ldots, \mathrm{F}) \rightarrow \mathrm{F} \text { । _ } \rightarrow \text { bot }_{\text {bool }}\right)\right\rangle \\
& \text { where }\left\{a_{1}, \ldots, a_{n}\right\}=\text { fn } e \cup\{b\} \text { and } b \# e \\
& \langle F, \text { the } x . e\rangle \rightarrow\left\langle F, \nu b . \text { case }_{\text {bool }}\left(e\left[a_{1} / x\right], e\left[a_{2} / x\right], \ldots, e\left[a_{n} / x\right], e[b / x]\right)\right. \text { of } \\
& \left.\left((\mathrm{T}, \mathrm{F}, \ldots, \mathrm{F}, \mathrm{F}) \rightarrow a_{1}\left|(\mathrm{~F}, \mathrm{~T}, \ldots, \mathrm{F}, \mathrm{F}) \rightarrow a_{2}\right| \ldots\left|(\mathrm{F}, \mathrm{F}, \ldots, \mathrm{T}, \mathrm{F}) \rightarrow a_{n}\right|{ }_{-} \rightarrow \text { bot }_{\text {name }}\right)\right\rangle \\
& \text { where }\left\{a_{1}, \ldots, a_{n}\right\}=\text { fn } e \text { and } b \# e
\end{aligned}
$$

Fig. 12. Frame-stack operational semantics of $\lambda \nu-\mathrm{PCF}^{+}$

$n$ ), whose proof uses $\langle F, e\rangle \rightarrow\left\langle F^{\prime}, e^{\prime}\right\rangle \wedge F^{\prime}\left[e^{\prime}\right] \Downarrow c \Rightarrow F[e] \Downarrow c$ (proved by case analysis of $\rightarrow$ ) and $F[e] \Downarrow c \Leftrightarrow e \Downarrow c^{\prime} \wedge F\left[c^{\prime}\right] \Downarrow c$ (proved by induction on $F$ ).

The frame-stack semantics specialises to $\lambda \nu-\mathrm{PCF}+$ the and $\lambda \nu-\mathrm{PCF}+\mathrm{ex}$ in the obvious way (by dismissing the rules for ex $x . e$ or the x.e), and Proposition A.5 remains to be valid for those languages. The fine-grainedness of the frame-stack rules allows us to formalise the intuitive justification of (27) in Section 6. 
Lemma A.6. Define for $n \in \mathbb{N}$ and $a \in \mathbb{A}$

$$
\begin{aligned}
& \text { eqBot }_{a, n} \triangleq \lambda x: \text { name } \rightarrow \nu b_{1} \ldots \nu b_{n} \text {. if }(x=a) \text { then } \mathrm{T} \text { else bot } \text { bool }_{\text {b }} \\
& E q B o t_{a} \triangleq\left\{\text { eqBot }_{a, n} \mid n \in \mathbb{N}\right\} \\
& \mathrm{kBot}_{n} \triangleq \lambda x: \text { name } \rightarrow \nu b_{1} \ldots \nu b_{n} \text {. } \text { bot }_{\text {bool }} \\
& K B o t \triangleq\left\{\operatorname{kBot}_{n} \mid n \in \mathbb{N}\right\}
\end{aligned}
$$

where $b_{1}, \ldots, b_{n}$ are distinct and fresh for $a$. Note that the definitions of eqBot ${ }_{a, n}$ and $\mathrm{kBot}_{n}$ are independent of the choice of $b_{1}, \ldots, b_{n}$ due to $\alpha$-equivalence. It then holds that

$$
\begin{aligned}
& (\forall i \in \mathbb{N})(\forall a \in \mathbb{A})(\forall j \in \mathbb{N})\left(\forall x_{1}, \ldots, x_{j} \in \mathbb{V}\right)\left(\forall e_{1}, \ldots, e_{j} \in E q B o t_{a}\right)(\forall \gamma \in\{\text { bool, nat, name }\}) \\
& \left(\forall\langle F, e\rangle \in \mathrm{Config}^{\lambda \nu \text {-PCF }+ \text { the }}\right)\left(\forall c \in \operatorname{Can}^{\lambda \nu \text {-PCF +the }}\right) a \notin \mathrm{fn}(\langle F, e\rangle) \wedge \\
& x_{1}: \text { name } \rightarrow \text { bool }, \ldots, x_{j}: \text { name } \rightarrow \text { bool } \vdash F[e]: \gamma \wedge\langle F, e\rangle\left[e_{1} / x_{1}, \ldots, e_{j} / x_{j}\right] \rightarrow^{i}\langle\mathrm{Id}, c\rangle \\
& \Rightarrow\left(\forall e_{1}^{\prime}, \ldots, e_{j}^{\prime} \in K B o t\right)\langle F, e\rangle\left[e_{1}^{\prime} / x_{1}, \ldots, e_{j}^{\prime} / x_{j}\right] \rightarrow^{*}\langle\mathrm{Id}, c\rangle .
\end{aligned}
$$

Proof. The proof works by induction on $i$. The $i=0$ case is obvious, because then $F=$ Id and $e=c$. For the inductive step we proceed by case distinction on the first transition. Special care needs to be taken in the case where $e$ is a variable, as this is where we need the generality of having multiple substitutions and name restrictions in the proof statement.

It is straight-forward to see that (56) is an instance of Lemma A.6. Similarly, we can use the frame-stack semantics to give a lemma that proves (57).

Lemma A.7. Define for any $n \in \mathbb{N}$

$$
\begin{aligned}
\mathrm{eq}_{a, n} & \triangleq \lambda x: \text { name } \rightarrow \nu b_{1} \ldots \nu b_{n} .(x=a) \\
E q_{a} & \triangleq\left\{\mathrm{eq}_{a, n} \mid n \in \mathbb{N}\right\}
\end{aligned}
$$

where $b_{1}, \ldots, b_{n}$ are distinct and not equal to a. Note that the definition of $\mathrm{eq}_{a, n}$ is independent of the choice of $b_{1}, \ldots, b_{n}$ due to $\alpha$-equivalence. It follows that:

$$
\begin{aligned}
& (\forall i \in \mathbb{N})(\forall a \in \mathbb{A})(\forall j \in \mathbb{N})\left(\forall x_{1}, \ldots, x_{j} \in \mathbb{V}\right)\left(\forall e_{1}, \ldots, e_{j} \in E q_{a}\right)\left(\forall\langle F, e\rangle \in \text { Config }^{\lambda \nu \text {-PCF }+ \text { ex }}\right) \\
& \left(\forall a^{\prime} \in \mathbb{A}\right) x_{1}: \text { name } \rightarrow \text { bool }, \ldots, x_{j}: \text { name } \rightarrow \text { bool } \vdash F[e]: \text { name } \wedge a \notin \operatorname{fn}(\langle F, e\rangle) \wedge \\
& \langle F, e\rangle\left[e_{1} / x_{1}, \ldots, e_{j} / x_{j}\right] \rightarrow^{i}\left\langle\operatorname{Id}, a^{\prime}\right\rangle \Rightarrow a \neq a^{\prime} .
\end{aligned}
$$

PROOF. We proceed by induction on $i$ and a case distinction on the first transition in the inductive step. The argument is very similar to the one in the proof of Lemma A.6, it only simplifies in many cases due to the simpler induction hypothesis.

\section{B. PROOF OF LEMMA 8.10}

We suppose given $\tau \in$ Typ, $u \in \mathrm{K} \llbracket \tau \rrbracket, A \subseteq_{\mathrm{f}} \mathbb{A}$ and a $\lambda \nu-\mathrm{PCF}^{+}$-expression $\emptyset \vdash e: \tau \rightarrow$ bool such that $\llbracket e \rrbracket=(u \searrow$ true $)$. We have to find $\emptyset \vdash e^{\prime}: \tau \rightarrow$ bool such that

$$
\llbracket e^{\prime} \rrbracket=\bigsqcup \operatorname{hull}_{A}\{(u \searrow \text { true })\} .
$$

Let Perm be the set of all finite permutations on $\mathbb{A}$, and define for each $f \in \operatorname{Perm} \rightarrow 2_{\perp}$ the analogue of (11) for permutations:

$$
\text { exists }_{\text {Perm }} f \triangleq \begin{cases}\text { true } & \text { if }(\exists \pi \in \text { Perm }) f \pi=\text { true } \\ \text { false } & \text { if }(\forall \pi \in \text { Perm }) f \pi=\text { false } \\ \perp & \text { otherwise. }\end{cases}
$$


This allows us to characterise $\bigsqcup \operatorname{hull}_{A}\{(u \searrow$ true $)\}$ by

$$
\begin{aligned}
& \bigsqcup \operatorname{hull}_{A}\{(u \searrow \text { true })\}=\lambda d \in \llbracket \tau \rrbracket \rightarrow \begin{cases}\text { true } & \text { if }(\exists \pi \in \text { Perm }) \pi \# A \wedge \pi \cdot u \sqsubseteq d \\
\perp & \text { otherwise }\end{cases} \\
& =\lambda d \in \llbracket \tau \rrbracket \rightarrow \text { exists }_{\text {Perm }}(\lambda \pi \in \text { Perm } \rightarrow \pi \# A \wedge(\pi \cdot u \searrow \text { true }) d) .
\end{aligned}
$$

Notation B.1. We write vectors of atomic names as $\vec{a}=a_{1}, \ldots, a_{n}$ and define the swapping of two vectors of the same length by

$$
(\vec{a} \vec{b}) \triangleq\left(a_{1} b_{1}\right) \circ \cdots \circ\left(a_{n} b_{n}\right) .
$$

Let $\mathbb{A}^{\# n}$ be the set of vectors of length $n$ that contain distinct atomic names. When convenient we confuse notation for vectors and sets, and write for example $\operatorname{supp} x=\vec{a}$.

The quantification over permutations and the permutation action in (60) can be encoded with quantification over atomic names and swappings:

Lemma B.2. Suppose $A \subseteq_{\mathrm{f}} \mathbb{A}, \pi \in$ Perm and $\pi \#$ A. For any element $u$ of a nominal set, suppose $\operatorname{supp} u-A=\vec{a}$ with $\vec{a} \in \mathbb{A}^{\# n}$, and let $b_{i}=\pi\left(a_{i}\right)$ for $i=1, \ldots, n$. Note that $\vec{b} \in \mathbb{A}^{\# n}$ (because $\pi$ is a permutation) and $\vec{b} \# A$ (because $\pi \# A$ and $a_{i} \notin A$ ). Picking any $\vec{c} \in \mathbb{A}^{\# n}$ with $\vec{c} \# A, \vec{a}, \vec{b}$, we get $\pi \cdot u=(\vec{b} \vec{c}) \circ(\vec{a} \vec{c}) \cdot u$.

Proof. It is easy to check that $\left(\pi^{-1} \circ(\vec{b} \vec{c}) \circ(\vec{a} \vec{c})\right) a=a$ holds for all $a \in \operatorname{supp} u$. Then the defining property (4) of $\operatorname{supp} u$ gives us $\left(\pi^{-1} \circ(\vec{b} \vec{c}) \circ(\vec{a} \vec{c})\right) \cdot u=u$, from which the desired property follows by applying $\pi$ to both sides of the equation.

Applying this Lemma B.2 to (60), together with the semantics of locally scoped names, it follows that (58) holds with

$$
\begin{gathered}
e^{\prime} \triangleq \lambda x: \tau \rightarrow \operatorname{ex} y_{1} \ldots \text { ex } y_{n} .(\text { distinct } \vec{y}) \text { and }(\vec{y} \text { freshfor } A) \\
\quad \text { and } \nu b_{1} \ldots \nu b_{n} .(((\vec{y} \rightleftharpoons \vec{b})(\vec{a} \rightleftharpoons \vec{b}) e) x)
\end{gathered}
$$

where $\llbracket e \rrbracket=(u \searrow$ true $)$ as above, $\vec{a} \triangleq \operatorname{supp} u-A$ and $\vec{b}$ are distinct atomic names satisfying $\vec{b} \# u, A$. For better readability, we used some syntactic sugar in the above expression:

- swapping vectors of expressions, $\left(\vec{e} \rightleftharpoons \overrightarrow{e^{\prime}}\right) \triangleq\left(e_{1} \rightleftharpoons e_{1}^{\prime}\right) \ldots\left(e_{n} \rightleftharpoons e_{n}^{\prime}\right)$

- boolean conjunction, $e_{1}$ and $e_{2} \triangleq$ if $e_{1}$ then $e_{2}$ else $\mathrm{F}$

- the expression distinct $\vec{e}$ stands for the expression that tests if all atomic names that $\vec{e}$ evaluates to are distinct

$-\vec{e}$ freshfor $A$ expresses that none of the expressions in the vector $\vec{e}$ evaluate to elements of the finite set $A \subseteq_{\mathrm{f}} \mathbb{A}$.

The last two constructs can be defined for any length of $\vec{e}$ by using conditionals and name equality tests.

\section{PROOF OF LEMMA 8.11}

Suppose we are given $\tau \in$ Typ such that

$$
\left(\forall v, v^{\prime} \in \mathrm{K} \llbracket \tau \rrbracket\right) v \ngtr v^{\prime} \Rightarrow(\exists e \in \operatorname{Exp}) \emptyset \vdash e: \tau \rightarrow \text { bool } \wedge \llbracket e \rrbracket=(v \searrow \text { true }) \sqcup\left(v^{\prime} \searrow \text { false }\right)
$$

where $v \forall v^{\prime}$ is notation for $v$ and $v^{\prime}$ not having an upper bound in $\llbracket \tau \rrbracket$. We also suppose given $u, u^{\prime} \in \mathrm{K} \llbracket \tau \rrbracket$ and $A \subseteq_{\mathrm{f}} \mathbb{A}$ with

$$
(\forall \pi \in \operatorname{Perm}) \pi \# A \Rightarrow u \forall \pi \cdot u^{\prime} .
$$


We have to find $\emptyset \vdash e: \tau \rightarrow$ bool such that

$$
\llbracket e \rrbracket=\bigsqcup \operatorname{hull}_{A}\left\{(u \searrow \text { true }),\left(u^{\prime} \searrow \text { false }\right)\right\} .
$$

Without loss of generality we may assume

$$
\left(\operatorname{supp} u \cap \operatorname{supp} u^{\prime}\right)-A=\emptyset
$$

because otherwise we can take $u^{\prime \prime} \triangleq(\vec{a} \vec{b}) \cdot u$ instead of $u^{\prime}$, where $\vec{a} \triangleq\left(\operatorname{supp} u \cap \operatorname{supp} u^{\prime}\right)-A$ and $\vec{b}$ are some distinct and fresh atomic names. (We use notation for swapping lists of atomic names as in Notation B.1.) It is easy to show that ( $\left.\operatorname{supp} u \cap \operatorname{supp} u^{\prime \prime}\right)-A=\emptyset$. We can replace $u^{\prime}$ with $u^{\prime \prime}$ because $(\vec{a} \vec{b}) \# A$ implies that $\bigsqcup$ hull ${ }_{A}\left\{(u \searrow\right.$ true $),\left(u^{\prime} \searrow\right.$ false $\left.)\right\}=$ $\sqcup$ hull $_{A}\left\{(u \searrow\right.$ true $),\left(u^{\prime \prime} \searrow\right.$ false $\left.)\right\}$ and that (61) as well as (62) hold for $u^{\prime \prime}$.

Note that (62) entails that whenever there is a $\pi \# A$ with $\pi \cdot u \sqsubseteq x$, then for all $\pi^{\prime} \# A$ it is the case that $\pi^{\prime} \cdot u \nsubseteq x$ (and symmetrically with $u$ and $\overline{u^{\prime}}$ interchanged). Therefore we get:

$$
\begin{aligned}
& \sqcup \text { hull }_{A}\left\{(u \searrow \text { true }),\left(u^{\prime} \searrow \text { false }\right)\right\} \\
& =\lambda d \in \llbracket \tau \rrbracket \rightarrow \begin{cases}\text { true } & \text { if }(\exists \pi \in \text { Perm }) \pi \# A \wedge \pi \cdot u \sqsubseteq d \\
\text { false } & \text { if }\left(\exists \pi^{\prime} \in \text { Perm }\right) \pi^{\prime} \# A \wedge \pi^{\prime} \cdot u^{\prime} \sqsubseteq d \\
\perp & \text { otherwise }\end{cases} \\
& =\lambda d \in \llbracket \tau \rrbracket \rightarrow \\
& \qquad \begin{cases}\text { true } & \text { if }(\exists \pi \in \text { Perm }) \pi \# A \wedge \pi \cdot u \sqsubseteq d \wedge\left(\forall \pi^{\prime} \in \text { Perm }\right) \pi^{\prime} \# A \Rightarrow \pi^{\prime} \cdot u^{\prime} \nsubseteq d \\
\text { false } & \text { if }\left(\exists \pi^{\prime} \in \text { Perm }\right) \pi^{\prime} \# A \wedge \pi^{\prime} \cdot u^{\prime} \sqsubseteq d \wedge(\forall \pi \in \text { Perm }) \pi \# A \Rightarrow \pi \cdot u \nsubseteq d \\
\perp & \text { otherwise }\end{cases} \\
& =\lambda d \in \llbracket \tau \rrbracket \rightarrow \text { exists } \text { Perm }\left(\lambda \pi \in \text { Perm } \rightarrow \pi \# A \wedge \text { all } \operatorname { P e r m } \left(\lambda \pi^{\prime} \in \text { Perm } \rightarrow \pi^{\prime} \# A\right.\right. \\
& \left.\left.\quad \Rightarrow\left((\pi \cdot u \searrow \text { true }) \sqcup\left(\pi^{\prime} \cdot u^{\prime} \searrow \text { false }\right)\right) d\right)\right)
\end{aligned}
$$

where

$$
a l l_{\text {Perm }} f \triangleq \begin{cases}\text { true } & \text { if }(\forall \pi \in \text { Perm }) f \pi=\text { true } \\ \text { false } & \text { if }(\exists \pi \in \text { Perm }) f \pi=\text { false } \\ \perp & \text { otherwise }\end{cases}
$$

is the dual of (59).

As in Appendix B, we can replace the quantifications over $\pi$ and $\pi^{\prime}$ in (65) by multiple quantifications over atomic names. Suppose supp $u-A$ consists of the distinct atomic names $\vec{b}=\left(b_{1}, \ldots, b_{n}\right) \in \mathbb{A}^{\# n}$ and $\operatorname{supp} u^{\prime}-A$ consists of the distinct atomic names $\vec{c}=\left(c_{1}, \ldots, c_{m}\right) \in \mathbb{A}^{\# m}$; so from (64) we have $\vec{b} \# \vec{c}$. Given $\pi, \pi^{\prime} \# A$, as in Lemma B.2 we have

$$
\begin{aligned}
\pi \cdot u & =\left(\overrightarrow{b^{\prime}} \vec{d}\right) \circ(\vec{b} \vec{d}) \cdot u \\
\pi^{\prime} \cdot u^{\prime} & =\left(\overrightarrow{c^{\prime}} \overrightarrow{d^{\prime}}\right) \circ\left(\vec{c} \overrightarrow{d^{\prime}}\right) \cdot u^{\prime}
\end{aligned}
$$

with $\vec{b}^{\prime}=\pi \cdot \vec{b}, \vec{c}^{\prime}=\pi^{\prime} \cdot \vec{c}$ and $\vec{d}, \vec{d}^{\prime}$ chosen suitably fresh. Therefore in view of (65), to solve (63) we can take $e$ to be

$$
\begin{aligned}
& e \triangleq \lambda x: \tau \rightarrow \operatorname{ex} x_{1} \ldots \operatorname{ex} x_{n} .(\operatorname{distinct} \vec{x}) \text { and }(\vec{x} \text { freshfor } A) \\
& \quad \text { and not }\left(\operatorname{ex} y_{1} \ldots \operatorname{ex} y_{m} .(\operatorname{distinct} \vec{y}) \text { and }(\vec{y} \text { freshfor } A) \text { and not }\left(e^{\prime} x\right)\right)
\end{aligned}
$$

provided we can find an expression $e^{\prime}$ with typing $x_{1}:$ name, $\ldots, x_{n}:$ name, $y_{1}$ : name $, \ldots, y_{m}:$ name $\vdash e^{\prime}: \tau \rightarrow$ bool that satisfies for each valuation $\rho$ that

$$
\llbracket e^{\prime} \rrbracket \rho=((\llbracket \vec{x} \rrbracket \rho \vec{d}) \circ(\vec{b} \vec{d}) \cdot u \searrow \text { true }) \sqcup\left(\left(\llbracket \vec{y} \rrbracket \rho \overrightarrow{d^{\prime}}\right) \circ\left(\vec{c} \overrightarrow{d^{\prime}}\right) \cdot u^{\prime} \searrow \text { false }\right)
$$


for some/any fresh $\vec{d}, \overrightarrow{d^{\prime}}$. In (66) we use the syntactic sugar from Appendix B as well as not $e \triangleq$ if $e$ then $\mathrm{F}$ else $\mathrm{T}$.

Giving such an $e^{\prime}$ might seem easy at first, since in view of (61) and (62), $(\pi \cdot u \searrow$ true) $\sqcup\left(\pi^{\prime} \cdot u^{\prime} \searrow\right.$ false $)$ is $\lambda \nu$-PCF-definable for any particular $\pi, \pi^{\prime} \# A$. However, it is not so easy: the problem is that $e^{\prime}$ has $\vec{x}, \vec{y}$ as free variables and we need to be parametric with respect to whatever atomic names those free variables get assigned by a given valuation $\rho$. Specifically, to define $e^{\prime}$ we need to consider all ways in which atomic names assigned to $\vec{x}$ and $\vec{y}$ may overlap. Fortunately each way corresponds to a partial bijection from $\{1, \ldots, n\}$ to $\{1, \ldots, m\}$ and there are only finitely many of them, $N$ say. Thus we take

$$
\begin{aligned}
& e^{\prime} \triangleq \text { if not }\left(x_{1}=y_{1}\right) \text { and } \operatorname{not}\left(x_{1}=y_{2}\right) \text { and } \ldots \text { and } \operatorname{not}\left(x_{n}=y_{m}\right) \text { then } e_{1} \\
& \quad \text { else if } x_{1}=y_{1} \text { and } \operatorname{not}\left(x_{2}=y_{2}\right) \text { and } \ldots \text { and } \operatorname{not}\left(x_{n}=y_{m}\right) \text { then } e_{2} \\
& \quad \vdots \\
& \quad \text { else if } x_{1}=y_{1} \text { and } x_{2}=y_{2} \text { and } \ldots \text { and } x_{n}=y_{m} \text { then } e_{N} \\
& \quad \text { else } \lambda(x: \tau) \rightarrow \mathrm{F}
\end{aligned}
$$

and show how to define the expressions $e_{1}, \ldots, e_{N}$ in such a way that for each $\rho$, $\llbracket e^{\prime} \rrbracket \rho=$ $\llbracket e_{i} \rrbracket \rho$ for the $i$ corresponding to the overlap conditions $\rho$ induces between $\vec{x}$ and $\vec{y}$. Then (67) holds because the if-clauses in (68) are exhaustive; in particular the $\lambda(x: \tau) \rightarrow \mathrm{F}$ case will never be reached. Let $f_{i}$ be the partial bijection corresponding to $e_{i}$, and define $\overrightarrow{b_{i}} \triangleq\left\{b_{j} \mid j \in \operatorname{dom} f_{i}\right\}, \overrightarrow{c_{i}} \triangleq\left\{c_{f_{i}(j)} \mid j \in \operatorname{dom} f_{i}\right\}, \overrightarrow{c_{i}^{\prime}} \triangleq\left\{c_{j} \mid j \notin \operatorname{im} f_{i}\right\}, \overrightarrow{x_{i}} \triangleq\left\{x_{j} \mid j \in \operatorname{dom} f_{i}\right\}$, $\overrightarrow{y_{i}} \triangleq\left\{y_{f_{i}(j)} \mid j \in \operatorname{dom} f_{i}\right\}, \overrightarrow{y_{i}^{\prime}} \triangleq\left\{y_{j} \mid j \notin \operatorname{im} f_{i}\right\}$. By (61) let $\emptyset \vdash e_{i}^{\prime}: \tau \rightarrow$ bool be given such that $\llbracket e_{i}^{\prime} \rrbracket=(u \searrow$ true $) \sqcup\left(\left(\overrightarrow{c_{i}} \overrightarrow{b_{i}}\right) \cdot u^{\prime} \searrow\right.$ false $)$. This allows us to define

$$
e_{i} \triangleq \nu d_{1} \ldots \nu d_{n} . \nu d_{1}^{\prime} \ldots \nu d_{m-\left|\operatorname{dom} f_{i}\right|}^{\prime} \cdot\left(\overrightarrow{y_{i}^{\prime}} \rightleftharpoons \overrightarrow{d^{\prime}}\right)(\vec{x} \rightleftharpoons \vec{d})\left(\overrightarrow{c_{i}^{\prime}} \rightleftharpoons \overrightarrow{d^{\prime}}\right)(\vec{b} \rightleftharpoons \vec{d}) e_{i}^{\prime}
$$

where $\vec{d}, \overrightarrow{d^{\prime}}$ consist of distinct atomic names that satisfy $\vec{d}, \overrightarrow{d^{\prime}} \# A, \vec{b}, \vec{c}$; through $\alpha$ renaming they may also be chosen to satisfy $\vec{d}, \overrightarrow{d^{\prime}} \# \llbracket \vec{x} \rrbracket \rho, \llbracket \vec{y} \rrbracket \rho$ once we are given a particular valuation $\rho$. Define also $\vec{d}_{i}=\left\{d_{j} \mid j \in \operatorname{dom} f_{i}\right\}$. Finally, with $\pi \cdot((u \searrow$ true $) \sqcup$ $\left(u^{\prime} \searrow\right.$ false $\left.)\right)=(\pi \cdot u \searrow$ true $) \sqcup\left(\pi \cdot u^{\prime} \searrow\right.$ false $)$, property (31),

$$
\begin{aligned}
& \left(\llbracket \overrightarrow{y_{i}^{\prime}} \rrbracket \rho \overrightarrow{d^{\prime}}\right) \circ(\llbracket \vec{x} \rrbracket \rho \vec{d}) \circ\left(\overrightarrow{c_{i}^{\prime}} \overrightarrow{d^{\prime}}\right) \circ(\vec{b} \vec{d}) \cdot u \\
& =\left(\llbracket \overrightarrow{y_{i}^{\prime}} \rrbracket \rho \overrightarrow{d^{\prime}}\right) \circ(\llbracket \vec{x} \rrbracket \rho \vec{d}) \circ(\vec{b} \vec{d}) \cdot u \\
& =(\llbracket \vec{x} \rrbracket \rho \vec{d}) \circ(\vec{b} \vec{d}) \cdot u
\end{aligned}
$$

$$
\begin{aligned}
& \text { as }\left(\overrightarrow{c_{i}^{\prime}} \overrightarrow{d^{\prime}}\right) \# \vec{d} \\
& \text { as }\left(\llbracket \overrightarrow{y_{i}^{\prime}} \rrbracket \rho \overrightarrow{d^{\prime}}\right) \# \llbracket \vec{x} \rrbracket \rho
\end{aligned}
$$

and

$$
\begin{aligned}
& \left(\llbracket \overrightarrow{y_{i}^{\prime}} \rrbracket \rho \overrightarrow{d^{\prime}}\right) \circ(\llbracket \vec{x} \rrbracket \rho \vec{d}) \circ\left(\overrightarrow{c_{i}^{\prime}} \overrightarrow{d^{\prime}}\right) \circ(\vec{b} \vec{d}) \circ\left(\overrightarrow{c_{i}} \overrightarrow{b_{i}}\right) \cdot u^{\prime} \\
& =\left(\llbracket \overrightarrow{y_{i}^{\prime}} \rrbracket \rho \overrightarrow{d^{\prime}}\right) \circ(\llbracket \vec{x} \rrbracket \rho \vec{d}) \circ\left(\overrightarrow{c_{i}^{\prime}} \overrightarrow{d^{\prime}}\right) \circ\left(\overrightarrow{c_{i}} \overrightarrow{d_{i}}\right) \circ(\vec{b} \vec{d}) \cdot u^{\prime} \quad \text { as } \pi \circ(a b)=(\pi a \pi b) \circ \pi \\
& =\left(\llbracket \overrightarrow{y_{i}^{\prime}} \rrbracket \rho \overrightarrow{d^{\prime}}\right) \circ(\llbracket \vec{x} \rrbracket \rho \vec{d}) \circ\left(\overrightarrow{c_{i}^{\prime}} \overrightarrow{d^{\prime}}\right) \circ\left(\overrightarrow{c_{i}} \overrightarrow{d_{i}}\right) \cdot u^{\prime} \quad \text { as }(\vec{b} \vec{d}) \# u^{\prime} \\
& =\left(\llbracket \overrightarrow{y_{i}^{\prime}} \rrbracket \rho \overrightarrow{d^{\prime}}\right) \circ\left(\llbracket \overrightarrow{x_{i}} \rrbracket \rho \overrightarrow{d_{i}}\right) \circ\left(\overrightarrow{c_{i}^{\prime}} \overrightarrow{d^{\prime}}\right) \circ\left(\overrightarrow{c_{i}} \overrightarrow{d_{i}}\right) \cdot u^{\prime} \quad \text { as } \vec{x}=\overrightarrow{x_{i}} \cup \overrightarrow{x_{i}^{\prime}} \\
& =\left(\llbracket \overrightarrow{y_{i}^{\prime}} \rrbracket \rho \overrightarrow{d^{\prime}}\right) \circ\left(\llbracket \overrightarrow{y_{i}} \rrbracket \rho \overrightarrow{d_{i}}\right) \circ\left(\overrightarrow{c_{i}^{\prime}} \overrightarrow{d^{\prime}}\right) \circ\left(\overrightarrow{c_{i}} \overrightarrow{d_{i}}\right) \cdot u^{\prime} \quad \text { as } \llbracket \overrightarrow{x_{i}} \rrbracket \rho=\llbracket \overrightarrow{y_{i}} \rrbracket \rho \\
& =\left(\llbracket \vec{y} \rrbracket \rho \overrightarrow{d^{\prime \prime}}\right) \circ\left(\vec{c} \overrightarrow{d^{\prime \prime}}\right) \cdot u^{\prime} \quad \text { as } \vec{y}=\overrightarrow{y_{i}} \cup \overrightarrow{y_{i}^{\prime}} \text {, with } \overrightarrow{d^{\prime \prime}}=\overrightarrow{d^{\prime}} \cup \overrightarrow{d_{i}}
\end{aligned}
$$

we obtain that $\llbracket e_{i} \rrbracket \rho=\llbracket e^{\prime} \rrbracket \rho$ as required.

This concludes the proof of Lemma 8.11. Since the last part of the proof is combinatorially complicated, we illustrate the constructions for a simple instance. 
Example C.1. Suppose we are in the special case of Lemma 8.11 where $A=\{a\}$, $\operatorname{supp} u=\left\{b_{1}, b_{2}\right\}$ and $\operatorname{supp} u^{\prime}=\left\{c_{1}, c_{2}\right\}$. In this setting, the expression $e$ satisfying $\llbracket e \rrbracket=\bigsqcup \operatorname{hull}_{\{a\}}\left\{(u \searrow\right.$ true $),\left(u^{\prime} \searrow\right.$ false $\left.)\right\}$ is defined by

$$
\begin{aligned}
& e \triangleq \lambda x: \tau \rightarrow \text { ex } x_{1} \cdot \operatorname{ex} x_{2} \cdot \operatorname{not}\left(x_{1}=x_{2}\right) \text { and } \operatorname{not}\left(x_{1}=a\right) \text { and } \operatorname{not}\left(x_{2}=a\right) \\
& \quad \text { and } \operatorname{not}\left(\operatorname{ex} y_{1} \cdot \operatorname{ex} y_{2} \cdot \operatorname{not}\left(x_{1}=x_{2}\right) \text { and } \operatorname{not}\left(y_{1}=a\right) \text { and } \operatorname{not}\left(y_{2}=a\right) \text { and } \operatorname{not}\left(e^{\prime} x\right)\right)
\end{aligned}
$$

where the expression $e^{\prime}$ reads

$$
\begin{aligned}
e^{\prime} \triangleq & \text { if not }\left(x_{1}=y_{1}\right) \text { and } \operatorname{not}\left(x_{2}=y_{2}\right) \text { and } \operatorname{not}\left(x_{1}=y_{2}\right) \text { and } \operatorname{not}\left(x_{n}=y_{m}\right) \text { then } e_{1} \\
& \text { else if } x_{1}=y_{1} \text { and } \operatorname{not}\left(x_{2}=y_{2}\right) \text { then } e_{2} \text { else if } \operatorname{not}\left(x_{1}=y_{1}\right) \text { and } x_{2}=y_{2} \text { then } e_{3} \\
& \text { else if } x_{1}=y_{2} \text { and } \operatorname{not}\left(x_{2}=y_{1}\right) \text { then } e_{4} \text { else if } \operatorname{not}\left(x_{1}=y_{2}\right) \text { and } x_{2}=y_{1} \text { then } e_{5} \\
& \text { else if } x_{1}=y_{1} \text { and } x_{2}=y_{2} \text { then } e_{6} \text { else if } x_{1}=y_{2} \text { and } x_{2}=y_{1} \text { then } e_{7} \\
& \text { else } \lambda x: \tau \rightarrow \mathrm{F} .
\end{aligned}
$$

Compare this to (66) and (68). Let us now define $e_{3}$ explicitly as in (69). The corresponding partial bijection is $f_{3}=\{2 \mapsto 2\}$ and by (61) we may assume the existence of $e_{3}^{\prime}$ with $\llbracket e_{3}^{\prime} \rrbracket=(u \searrow$ true $) \sqcup\left(\left(c_{2} b_{2}\right) \cdot u^{\prime} \searrow\right.$ false $)$. Then we can define

$$
e_{3} \triangleq \nu d_{1} \cdot \nu d_{2} \cdot \nu d_{1}^{\prime} \cdot\left(y_{1} \rightleftharpoons d_{1}^{\prime}\right)\left(x_{1} \rightleftharpoons d_{1}\right)\left(x_{2} \rightleftharpoons d_{2}\right)\left(c_{1} \rightleftharpoons d_{1}^{\prime}\right)\left(b_{1} \rightleftharpoons d_{1}\right)\left(b_{2} \rightleftharpoons d_{2}\right) e_{3}^{\prime}
$$

for which $\llbracket e_{3} \rrbracket \rho=\left(\left(\llbracket x_{1} \rrbracket \rho d_{1}\right) \circ\left(\llbracket x_{2} \rrbracket \rho d_{2}\right) \circ\left(b_{1} d_{1}\right) \circ\left(b_{2} d_{2}\right) \cdot u \searrow\right.$ true $) \sqcup\left(\left(\llbracket y_{1} \rrbracket \rho d_{1}^{\prime}\right) \circ\left(\llbracket y_{2} \rrbracket \rho d_{2}\right) \circ\right.$ $\left(c_{1} d_{1}^{\prime}\right) \circ\left(c_{2} d_{2}\right) \cdot u^{\prime} \searrow$ false $)$ holds for any $\rho$ under the conditions that $\llbracket x_{1} \rrbracket \rho \neq \llbracket y_{1} \rrbracket \rho$ and $\llbracket x_{2} \rrbracket \rho=\llbracket y_{2} \rrbracket \rho$.

\section{PROOF OF THEOREM 11.2}

The overall structure of the proof of Theorem 11.2 is the same as in Theorem 7.5. We extend the logical relation to cover the type of $\lambda$-terms and abstraction types:

$$
\begin{aligned}
& d \triangleleft_{\text {term }} e \triangleq d=\perp \vee(\exists c) e \Downarrow c \wedge \llbracket c \rrbracket=d \\
& d \triangleleft_{\delta \tau} e \triangleq\left(\text { Иa)d@a } a \triangleleft_{\tau} e @ a .\right.
\end{aligned}
$$

In the second clause we use the freshness quantifier $(И a)$ of nominal logic [Pitts 2013, Section 3.2]; thus $d \triangleleft_{\delta \tau} e$ holds if and only if $d @ a \triangleleft_{\tau} e @ a$ holds for some $a \#(d, e)$, or equivalently, for any $a \#(d, e)$.

The proof that this logical relation is closed under restriction, abstraction and concretion

$$
\begin{aligned}
d \triangleleft_{\tau} e & \Rightarrow(\forall a) a \backslash d \triangleleft_{\tau} \nu a . e \\
d \triangleleft_{\tau} e & \Rightarrow(\forall a)\langle a\rangle d \triangleleft_{\delta \tau} \alpha a . e \\
d \triangleleft_{\delta \tau} e & \Rightarrow(\forall a) d @ a \triangleleft_{\tau} e @ a
\end{aligned}
$$

is not straightforward and occupies the rest of this appendix. Using these three properties one can then prove the fundamental property of the logical relation as in Theorem 7.5 , and computational adequacy follows from that in the usual way.

To prove (70)-(72) we introduce a weaker form of 'Kleene preorder' between expressions:

Definition D.1. For every PNA type $\tau \in$ Typ, let $\operatorname{Exp}(\tau) \triangleq\{e \in \operatorname{Exp} \mid \emptyset \vdash e: \tau\}$ and $\operatorname{Can}(\tau) \triangleq\{c \in \operatorname{Can} \mid \emptyset \vdash c: \tau\}$, and define the weak Kleene preorders

$$
\begin{aligned}
& \varliminf_{\tau}^{\mathrm{wk}} \subseteq \operatorname{Exp}(\tau) \times \operatorname{Exp}(\tau) \\
& \leq_{\tau}^{\mathrm{wk}} \subseteq \operatorname{Can}(\tau) \times \operatorname{Can}(\tau)
\end{aligned}
$$


by simultaneously structural recursion over $\tau$ :

$$
\begin{aligned}
& e \leqq_{\tau}^{\mathrm{wk}} e^{\prime} \triangleq(\forall c) e \Downarrow c \Rightarrow\left(\exists c^{\prime}\right) e^{\prime} \Downarrow c^{\prime} \wedge c \leq_{\tau}^{\mathrm{wk}} c^{\prime} \\
& c \leq_{\gamma}^{\mathrm{wk}} c^{\prime} \triangleq c=c^{\prime} \quad(\gamma \in\{\text { name, bool, nat, term }\}) \\
& c \leq_{\tau_{1} \times \tau_{2}}^{\mathrm{wk}} c^{\prime} \triangleq\left(\exists e_{1}, e_{2}, e_{1}^{\prime}, e_{2}^{\prime}\right) c=\left(e_{1}, e_{2}\right) \wedge c^{\prime}=\left(e_{1}^{\prime}, e_{2}^{\prime}\right) \wedge e_{1} \leqq_{\tau_{1}}^{\mathrm{wk}} e_{1}^{\prime} \wedge e_{2} \leqq_{\tau_{2}}^{\mathrm{wk}} e_{2}^{\prime} \\
& c \leq_{\tau_{1} \rightarrow \tau_{2}}^{\mathrm{wk}} c^{\prime} \triangleq\left(\exists x, e, e^{\prime}\right) c=\lambda x: \tau_{1} \rightarrow e \wedge c^{\prime}=\lambda x: \tau_{1} \rightarrow e^{\prime} \\
& \wedge\left(\forall e_{1} \in \operatorname{Exp}\left(\tau_{1}\right)\right) e\left[e_{1} / x\right] \leqq \tau_{2} e^{\prime}\left[e_{1} / x\right] \\
& c \leq_{\delta \tau}^{\mathrm{wk}} c^{\prime} \triangleq(И a)\left(\exists c_{1}, c_{1}^{\prime}\right) c=\alpha a \cdot c_{1} \wedge c^{\prime}=\alpha a \cdot c_{1}^{\prime} \wedge c_{1} \leq_{\tau}^{\mathrm{wk}} c_{1}^{\prime} .
\end{aligned}
$$

The next lemma collects together the properties of these preorders that we need.

LEMMA D.2. The following properties hold:

$$
\begin{aligned}
& \left((\forall c) e \Downarrow c \Rightarrow e^{\prime} \Downarrow c\right) \Rightarrow e \leqq_{\tau}^{\mathrm{wk}} e^{\prime} . \\
& c \leq_{\tau}^{\mathrm{wk}} c^{\prime} \wedge a\left\|c:=c_{1} \Rightarrow\left(\exists c_{1}^{\prime}\right) a\right\| c^{\prime}:=c_{1}^{\prime} \wedge c_{1} \leq_{\tau}^{\mathrm{wk}} c_{1}^{\prime} \\
& e \leqq_{\tau}^{\mathrm{wk}} e^{\prime} \Rightarrow \nu a . e \leqq_{\tau}^{\mathrm{wk}} \nu a . e^{\prime} \\
& e \leqq \delta \tau=e^{\mathrm{wk}} e^{\prime} \Rightarrow(\forall a) e @ a \leqq_{\tau}^{\mathrm{wk}} e^{\prime} @ a \\
& e \leqq_{\tau_{1} \times \tau_{2}}^{\mathrm{wk}} e^{\prime} \Rightarrow \text { fst } e \leqq_{\tau_{1}}^{\mathrm{wk}} \text { fst } e^{\prime} \wedge \text { snd } e \leqq_{\tau_{2}}^{\mathrm{wk}} \text { snd } e^{\prime} \\
& e \leqq\rfloor_{\tau_{1} \rightarrow \tau_{2}}^{\mathrm{wk}} e^{\prime} \Rightarrow\left(\forall e_{1} \in \operatorname{Exp}\left(\tau_{1}\right)\right) e e_{1} \varliminf_{\tau_{2}}^{\mathrm{wk}_{2}} e e_{1} \\
& a^{\prime} \backslash \backslash c:=c_{1} \wedge a\left\|c_{1}:=c_{2} \Rightarrow\left(\exists c_{1}^{\prime}, c_{2}^{\prime}\right) a\right\| c:=c_{1}^{\prime} \wedge a^{\prime} \backslash c_{1}^{\prime}:=c_{2}^{\prime} \wedge c_{2} \leq_{\tau}^{\mathrm{wk}} c_{2}^{\prime} \\
& \nu a . \nu a^{\prime} . e \varliminf_{\tau}^{\mathrm{wk}} \nu a^{\prime} . \nu a . e \\
& a \# c \Rightarrow\left(\exists c^{\prime}\right) a \| c:=c^{\prime} \wedge c \leq_{\tau}^{\mathrm{wk}} c^{\prime} \wedge c^{\prime} \leq_{\tau}^{\mathrm{wk}} c \\
& a \# e \Rightarrow \nu a . e \leqq_{\tau}^{\mathrm{wk}} e \wedge e \leqq_{\tau}^{\mathrm{wk}} \nu a . e \\
& a \neq a^{\prime} \Rightarrow \nu a .\left(e @ a^{\prime}\right) \varliminf_{\tau}^{\mathrm{wk}}(\nu a . e) @ a^{\prime} \\
& \nu a . \text { fst } e \leqq \leqq_{\tau}^{\mathrm{wk}} \text { fst }(\nu a . e) \\
& \left.\nu a . \text { snd } e \leqq_{\tau}^{\mathrm{wk}} \text { snd ( } \nu a . e\right) \\
& a \# e_{1} \Rightarrow \nu a .\left(e e_{1}\right) \leqq_{\tau}^{\mathrm{wk}}(\nu a . e) e_{1} \text {. }
\end{aligned}
$$

Proof. (73) follows from the definition of $\leq_{\tau}^{\mathrm{wk}}$ and the easily verified fact that $\leq_{\tau}^{\mathrm{wk}}$ is reflexive. (74) can be proved by induction on the structure of $\tau$ from the definition of $\leqq$ wk; and then (75) and (76) follow from this. The proof of (76) additionally uses that $\varliminf^{\mathrm{wk}}$ and $\leq \leq^{\mathrm{wk}}$ are equivariant, which is straight-forward to prove. (77) and (78) are direct consequences of the definition of $\leqq$ wk. (79) can be proved from the definition of $\leq$ wk by induction on the structure of $\tau$; and then (80) follow from this. Similarly for and (81) and (82). (83) follows from (79) and (80). (84)-(86) are immediate by using (73).

Corollary D.3.

$$
\begin{aligned}
& e \leqq_{\tau}^{\mathrm{wk}}(\alpha a . e) @ a \\
& a^{\prime} \#(a, e) \Rightarrow \nu a^{\prime} .\left(a a^{\prime}\right) \cdot\left(e @ a^{\prime}\right) \leqq_{\tau}^{\mathrm{wk}} e @ a
\end{aligned}
$$

PROOF. For showing that (88) implies (87), we use transitivity of $\leqq_{\tau}^{\text {wk }}$ and prove $e \leqq_{\tau}^{\mathrm{wk}} \nu a^{\prime} .\left(a a^{\prime}\right) \cdot\left(\alpha a . e @ a^{\prime}\right) \leqq_{\tau}^{\mathrm{wk}} \alpha a . e @ a$ for some/any $a^{\prime} \# a, e$. The second preorder relation is a consequence of (88), whereas the first one can be proved directly. 
For (88), suppose $a^{\prime} \#(a, e)$ and

$$
\nu a^{\prime} .\left(a a^{\prime}\right) \cdot\left(e @ a^{\prime}\right) \Downarrow c .
$$

We have to show $e @ a \Downarrow c^{\prime}$, for some $c^{\prime}$ with $c \leq_{\tau}^{\text {wk }} c^{\prime}$. But (89) can only hold because for some $c_{1}$ we have

$$
\begin{aligned}
& \left(a a^{\prime}\right) \cdot\left(e @ a^{\prime}\right) \Downarrow c_{1} \\
& a^{\prime} \backslash c_{1}:=c .
\end{aligned}
$$

From (90) we get (by equivariance of $\Downarrow$ ) that $e @ a^{\prime} \Downarrow\left(a a^{\prime}\right) \cdot c_{1}$; and hence we must have for some $a^{\prime \prime} \#\left(a, a^{\prime}, e, c, c_{1}\right)$ and $c_{2}$ that

$$
\begin{aligned}
& e \Downarrow \alpha a^{\prime \prime} . c_{2} \\
& a^{\prime \prime} \backslash\left(a^{\prime \prime} a^{\prime}\right) \cdot c_{2}:=\left(a a^{\prime}\right) \cdot c_{1} .
\end{aligned}
$$

Since $a^{\prime} \# e$, from (92) we get $a^{\prime} \# c_{2}$ and hence $a^{\prime \prime} \#\left(a^{\prime \prime} a^{\prime}\right) \cdot c_{2}$. Then by (81) and (93) we have $\left(a a^{\prime}\right) \cdot c_{1} \leq_{\tau}^{\mathrm{wk}}\left(a^{\prime \prime} a^{\prime}\right) \cdot c_{2}$ and hence also

$$
c_{1} \leq_{\tau}^{\mathrm{wk}}\left(a a^{\prime}\right)\left(a^{\prime \prime} a^{\prime}\right) \cdot c_{2} .
$$

Applying (74) to (91) and (94), there exists $c^{\prime}$ with

$$
a^{\prime} \backslash\left(a a^{\prime}\right)\left(a^{\prime \prime} a^{\prime}\right) \cdot c_{2}:=c^{\prime} \wedge c \leq_{\tau}^{\mathrm{wk}} c^{\prime} .
$$

Note that since $a^{\prime} \# c_{2}, \alpha a^{\prime \prime} . c_{2}=\alpha a^{\prime} .\left(a^{\prime \prime} a^{\prime}\right) \cdot c_{2}$ and therefore from (92), $e \Downarrow \alpha a^{\prime} .\left(a^{\prime \prime} a^{\prime}\right)$. $c_{2}$. Combining this with (95) we get $e @ a \Downarrow c^{\prime}$ and $c \leq_{\tau}^{\mathrm{wk}} c^{\prime}$, as required.

We can now complete the proof of properties (70)-(72) of the logical relation:

- (70) follows by structural induction on $\tau$, using (83) for name abstraction types, (84) and (85) for product types, and (86) for function types.

- (71) follows from (87).

- Finally, for (72), suppose $d \triangleleft_{\delta \tau} e$. Given any $a \in \mathbb{A}$, pick $a^{\prime} \#(a, d, e)$. Then putting $d^{\prime} \triangleq d @ a^{\prime}$, we have $d=\left\langle a^{\prime}\right\rangle d^{\prime}$ and $d @ a=a^{\prime} \backslash\left(a a^{\prime}\right) \cdot d^{\prime}$. By definition of $\triangleleft_{\delta \tau}$ we have $d^{\prime} \triangleleft_{\tau} e @ a^{\prime}$ and hence by equivariance of the logical relation, $\left(a a^{\prime}\right) \cdot d^{\prime} \triangleleft_{\tau}\left(a a^{\prime}\right) \cdot\left(e @ a^{\prime}\right)$. So by (70) and (88), $d @ a=a^{\prime} \backslash\left(a a^{\prime}\right) \cdot d^{\prime} \triangleleft_{\tau} \nu a^{\prime} .\left(a a^{\prime}\right) \cdot\left(e @ a^{\prime}\right) \varliminf_{\tau}^{\mathrm{wk}} e @ a$. So it just remains to see that the logical relation is closed under composition with $\leqq$ wk

$$
d \triangleleft_{\tau} e \wedge e \leqq_{\tau}^{\mathrm{wk}} e^{\prime} \Rightarrow d \triangleleft_{\tau} e^{\prime}
$$

which follows by combining (76)-(78) with the definitions of $\leqq$ wk and $\triangleleft$.

\section{REFERENCES}

S. Abramsky. 1991. Domain Theory In Logical Form. Annals of Pure and Applied Logic 51 (1991), 1-77.

S. Abramsky, D. R. Ghica, A. S. Murawski, C.-H. L. Ong, and I. D. B. Stark. 2004. Nominal Games and Full Abstraction for the Nu-Calculus. In Proc. LICS 2004. IEEE Computer Society Press, 150-159.

S. Abramsky, R. Jagadeesan, and P. Malacaria. 2000. Full Abstraction for PCF. Information and Computation 163, 2 (2000), 409-470.

S. Abramsky and A. Jung. 1994. Domain Theory. In Handbook of Logic in Computer Science, S. Abramsky, D. M. Gabbay, and T. S. E. Maibaum (Eds.). Vol. 3. Clarendon Press, 1-168.

M. Bojańczyk, L. Braud, B. Klin, and S. Lasota. 2012. Towards Nominal Computation. In Proc. POPL 2012. ACM Press, 401-412.

M. Bojańczyk, B. Klin, and S. Lasota. 2011. Automata with Group Actions. In Proc. LICS 2011. IEEE Computer Society Press, 355-364.

M. Bojańczyk and S. Lasota. 2012. A Machine-Independent Characterization of Timed Languages. In Proc. ICALP 2012, Part II (Lecture Notes in Computer Science), A. Czumaj, K. Mehlhorn, A. M. Pitts, and R. Wattenhofer (Eds.), Vol. 7392. Springer-Verlag, 92-103. 
V. Ciancia and U. Montanari. 2010. Symmetries, Local Names and Dynamic (De)-allocation of Names. Information and Computation 208, 12 (2010), 1349-1367.

P.-L. Curien. 2007. Definability and Full Abstraction. In Computation, Meaning and Logic, Articles dedicated to Gordon Plotkin, L. Cardelli, M. Fiore, and G. Winskel (Eds.). Electronic Notes in Theoretical Computer Science, Vol. 172. Elsevier, 301-310.

M. Felleisen and R. Hieb. 1992. The Revised Report on the Syntactic Theories of Sequential Control and State. Theoretical Computer Science 103 (1992), 235-271.

M. J. Gabbay. 2009. A Study of Substitution, Using Nominal Techniques and Fraenkel-Mostowski Sets. Theoretical Computer Science 410, 12-13 (2009), 1159-1189.

M. J. Gabbay. 2011. Foundations of Nominal Techniques: Logic and Semantics of Variables in Abstract Syntax. Bulletin of Symbolic Logic 17, 2 (2011), 161-229.

M. J. Gabbay and V. Ciancia. 2011. Freshness and Name-Restriction in Sets of Traces with Names. In Proc. FOSSACS 2011 (LNCS), Vol. 6604. Springer-Verlag, 365-380.

M. J. Gabbay and A. M. Pitts. 2002. A New Approach to Abstract Syntax with Variable Binding. Formal Aspects of Computing 13 (2002), 341-363.

P. Gabriel and F. Ulmer. 1971. Lokal Präsentierbare Kategorien. Lecture Notes in Mathematics, Vol. 221. Springer-Verlag.

F. Gadducci, M. Miculan, and U. Montanari. 2006. About Permutation Algebras, (Pre)sheaves and Named Sets. Higher-Order Symb. Computation 19 (2006), 283-304.

R. Harper. 2013. Practical Foundation for Programming Languages. Cambridge University Press.

J. M. E. Hyland and C.-H. L. Ong. 2000. On Full Abstraction for PCF: I, II and III. Information and Computation 163, 2 (2000), 285-408.

P. T. Johnstone. 2002. Sketches of an Elephant, A Topos Theory Compendium, Volumes 1 and 2. Number 43-44 in Oxford Logic Guides. Oxford University Press.

J. Laird. 2008. A Game Semantics of Names and Pointers. Annals of Pure and Applied Logic 151, 2 (2008), 151-169.

D. R. Licata and R. Harper. 2009. A Universe of Binding and Computation. In Proc. ICFP 2009. ACM, New York, NY, USA, 123-134.

S. Lösch and A. M. Pitts. 2011. Relating Two Semantics of Locally Scoped Names. In Proc. CSL 2011, Vol. 12. Schloss Dagstuhl-Leibniz-Zentrum für Informatik, 396-411.

S. Lösch and A. M. Pitts. 2013. Full Abstraction for Nominal Scott Domains. In Proc. POPL 2013. ACM Press, 3-14.

G. Markowsky. 1976. Chain-Complete Posets and Directed Sets with Applications. Algebra Universalis 6 (1976), 53-68.

E. Moggi. 1989. An Abstract View of Programming Languages. Technical Report ECS-LFCS-90-113. Department of Computer Science, University of Edinburgh.

U. Montanari and M. Pistore. 2000. $\pi$-Calculus, Structured Coalgebras and Minimal HD-Automata. In Proc. MFCS 2000 (LNCS), Vol. 1893. Springer-Verlag, 569-578.

A. S. Murawski and N. Tzevelekos. 2012. Algorithmic Games for Full Ground References. In Proc. ICALP 2012, Part II (LNCS), Vol. 7392. Springer-Verlag, 312-324.

M. Odersky. 1994. A Functional Theory of Local Names. In Proc. POPL 1994. ACM Press, 48-59.

D. L. Petrişan. 2011. Investigations into Algebra and Topology over Nominal Sets. Ph.D. Dissertation. Department of Computer Science, University of Leicester.

A. M. Pitts. 2002. Operational Semantics and Program Equivalence. In Applied Semantics, Advanced Lectures, International Summer School, APPSEM 2000, Caminha, Portugal, G. Barthe, P. Dybjer, and J. Saraiva (Eds.). Lecture Notes in Computer Science, Tutorial, Vol. 2395. Springer-Verlag, 378-412.

A. M. Pitts. 2006. Alpha-Structural Recursion and Induction. Journal of the ACM 53 (2006), 459-506.

A. M. Pitts. 2011. Structural Recursion with Locally Scoped Names. Journal of Functional Programming 21, 3 (2011), 235-286.

A. M. Pitts. 2013. Nominal Sets: Names and Symmetry in Computer Science. Cambridge Tracts in Theoretical Computer Science, Vol. 57. Cambridge University Press.

A. M. Pitts and M. J. Gabbay. 2000. A Metalanguage for Programming with Bound Names Modulo Renaming. In Proc. MPC 2000 (LNCS), Vol. 1837. Springer-Verlag, 230-255.

A. M. Pitts and I. D. B. Stark. 1993. Observable Properties of Higher Order Functions That Dynamically Create Local Names, or: What's new?. In Proc. MFCS 1993 (LNCS), Vol. 711. Springer-Verlag, 122-141.

G. D. Plotkin. 1977. LCF Considered as a Programming Language. Theoretical Computer Science 5 (1977), 223-255. 
F. Pottier. 2007. Static Name Control for FreshML. In Proc. LICS 2007. IEEE Computer Society Press, 356-365.

O. Savary-Belanger, S. Monnier, and B. Pientka. 2013. Programming Type-Safe Transformations Using Higher-Order Abstract Syntax. In Certified Programs and Proofs, G. Gonthier and M. Norrish (Eds.). Lecture Notes in Computer Science, Vol. 8307. Springer-Verlag, 243-258.

D. S. Scott. 1982. Domains for Denotational Semantics. In Proc. ICALP 1982 (LNCS), Vol. 140. SpringerVerlag, 577-610.

M. R. Shinwell. 2005. The Fresh Approach: Functional Programming with Names and Binders. Ph.D. Dissertation. University of Cambridge. Available as University of Cambridge Computer Laboratory Technical Report UCAM-CL-TR-618.

M. R. Shinwell and A. M. Pitts. 2005. On a Monadic Semantics for Freshness. Theoretical Computer Science 342 (2005), 28-55.

M. R. Shinwell, A. M. Pitts, and M. J. Gabbay. 2003. FreshML: Programming with Binders Made Simple. In Proc. ICFP 2003. ACM Press, 263-274.

I. D. B. Stark. 1994. Names and Higher-Order Functions. Ph.D. Dissertation. University of Cambridge. Available as University of Cambridge Computer Laboratory Technical Report Number UCAM-CL-TR363.

S. Staton. 2007. Name-Passing Process Calculi: Operational Models and Structural Operational Semantics. Ph.D. Dissertation. University of Cambridge. Available as University of Cambridge Computer Laboratory Technical Report Number UCAM-CL-TR-688.

T. Streicher. 2006. Domain-Theoretic Foundations of Functional Programming. World Scientific, Singapore.

D. C. Turner. 2009. Nominal Domain Theory for Concurrency. Ph.D. Dissertation. University of Cambridge. Available as University of Cambridge Computer Laboratory Technical Report UCAM-CL-TR-751.

D. C. Turner and G. Winskel. 2009. Nominal Domain Theory for Concurrency. In Proc. CSL 2009, E. Grädel and R. Kahle (Eds.). Lecture Notes in Computer Science, Vol. 5771. Springer-Verlag, 546-560.

N. Tzevelekos. 2008. Nominal Game Semantics. Ph.D. Dissertation. University of Oxford. Available as Oxford University Computing Laboratory Technical Report RR-09-18.

N. Tzevelekos. 2011. Fresh-Register Automata. In Proc. POPL 2011. ACM Press, 295-306.

N. Tzevelekos. 2012. Program Equivalence in a Simple Language with State. Computer Languages, Systems and Structures 38, 2 (2012), 181-198.

Received August 2013; revised February 2014; accepted April 2014 